University of San Diego

Digital USD

2001-06-01

\title{
Effect of Relaxation on Neuro-Immune Responses of Persons Undergoing Chemotherapy
}

Patricia K. Taylor Pothier PhD, MS, RN

University of San Diego

Follow this and additional works at: https://digital.sandiego.edu/dissertations

Part of the Nursing Commons

\section{Digital USD Citation}

Taylor Pothier, Patricia K. PhD, MS, RN, "Effect of Relaxation on Neuro-Immune Responses of Persons Undergoing Chemotherapy" (2001). Dissertations. 298.

https://digital.sandiego.edu/dissertations/298

This Dissertation: Open Access is brought to you for free and open access by the Theses and Dissertations at Digital USD. It has been accepted for inclusion in Dissertations by an authorized administrator of Digital USD. For more information, please contact digital@sandiego.edu. 


\title{
UNIVERSITY OF SAN DIEGO
}

Hahn School of Nursing and Health Science

DOCTOR OF PHILOSOPHY IN NURSING

\section{EFFECT OF RELAXATION ON NEURO-IMMUNE RESPONSES \\ OF PERSONS UNDERGOING CHEMOTHERAPY}

by

Patricia K. Taylor Pothier, MS, RN

\begin{abstract}
A dissertation presented to the
UNIVERSITY OF SAN DIEGO

In partial fulfillment of the

requirements for the degree

DOCTOR OF PHILOSOPHY IN NURSING
\end{abstract}

FACULTY OF THE HAHN SCHOOL OF NURSING AND HEALTH SCIENCE

June, 2001

Dissertation Committee

Janet K. Harrison, EdD, RN, Chairperson

Mary Jo Clark, PhD, RN

Lee S. Berk, DrPH, FACSM, CHES 


\section{ABSTRACT \\ Effect of Relaxation on Neuro-Immune Responses of Persons Undergoing Chemotherapy}

Chemotherapy can be considered both a physiclogic and a psychological stressor. Anxiety, nausea, emesis, and immunosuppression are commonly associated with chemotherapy, and may adversely affect the outcome or the process of treatment. Chemotherapy-related anxiety can exacerbate negative side-effects. Some psychological stressors, such as anxiety, can result in down-regulation of certain immune functions. Studies have demonstrated that behavioral interventions, such as specific relaxation strategies, can modify stress-related immune modulation. These behavioral interventions have also been shown to decrease the degree of nausea and vomiting associated with chemotherapy. The purpose of this study was to examine whether a relaxation intervention would result in decreased anxiety, decreased nausea and vomiting, and decreased immune modulation in persons undergoing chemotherapy for breast cancer.

A baseline neuro-immune assessment, including immune function tests, state and trait anxiety, and serum catecholamines, was performed on 12 women diagnosed with breast cancer and slated to undergo four cycles of Adriamycin/Cytoxan (AC) chemotherapy. Subjects were assigned to an experimental or a control group. The control group underwent chemotherapy according to the prescribed AC regimen. The experimental group underwent the same chemotherapy and a relaxation intervention. The neuro-immune assessment was repeated just before subjects received the third and fourth chemotherapy infusions, with the addition of assessment of nausea and emesis.

Study findings revealed that state anxiety was significantly lower at measure two for the experimental group $(t=3.20, p=0.02)$. No significant difference in anticipatory nausea and vomiting was found between groups. Evaluation of immune function tests was inconclusive. These findings suggest that a relaxation intervention may lower anxiety in individuals who are undergoing chemotherapy. Future research efforts should continue to explore whether relaxation interventions can modify the degree of side effects associated with chemotherapy, and potentially improve the quality of life for persons requiring the treatment. 


\section{ACKNOWLEDGMENTS}

It is with great appreciation that I acknowledge the members of my dissertation committee, Janet K. Harrison, EdD, RN, Mary Jo Clark, PhD, RN, and Lee S. Berk, DrPH, FACSM, CHES, for their expertise and guidance during the dissertation process. Special thanks goes to Dr. Harrison for her willingness to chair my committee, to Dr. Clark for taking time during her sabbatical for the reading and defense of my dissertation, and to $\mathrm{Dr}$. Berk for taking time from a busy research and teaching schedule to advise and mentor me.

I also wish to acknowledge the support and encouragement I received from the administration and from my colleagues and friends at Loma Linda University School of Nursing. The professional and financial support provided by the School of Nursing was invaluable in making this part of my education possible.

Finally my deepest appreciation goes to John Pothier, my husband and mainstay, and my parents, Dr. and Mrs. William Holmes Taylor for their wisdom and support. 


\section{TABLE OF CONTENTS}

Acknowledgments $\ldots \ldots \ldots \ldots \ldots \ldots \ldots \ldots \ldots \ldots \ldots \ldots \ldots \ldots \ldots \ldots \ldots \ldots$ ii

Chapter 1. Introduction

Introduction $\ldots \ldots \ldots \ldots \ldots \ldots \ldots \ldots \ldots \ldots \ldots \ldots \ldots \ldots \ldots \ldots \ldots \ldots \ldots$

Purpose $\ldots \ldots \ldots \ldots \ldots \ldots \ldots \ldots \ldots \ldots \ldots \ldots \ldots \ldots \ldots \ldots \ldots \ldots \ldots \ldots \ldots \ldots \ldots$

Background and Significance $\ldots \ldots \ldots \ldots \ldots \ldots \ldots \ldots \ldots \ldots \ldots \ldots \ldots \ldots \ldots \ldots \ldots \ldots$

Conceptual Framework $\ldots \ldots \ldots \ldots \ldots \ldots \ldots \ldots \ldots \ldots \ldots \ldots$

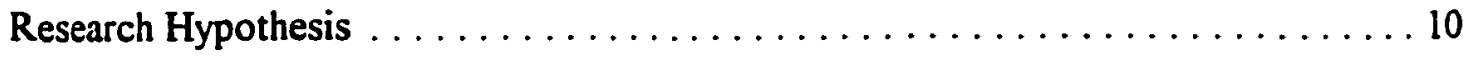

Definition of Major Variables or Concepts $\ldots \ldots \ldots \ldots \ldots \ldots \ldots \ldots \ldots$

Dependent Variables $\ldots \ldots \ldots \ldots \ldots \ldots \ldots \ldots \ldots \ldots \ldots \ldots \ldots$

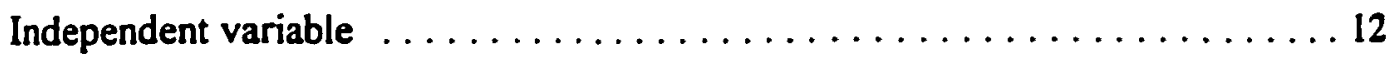

Attribute Variables $\ldots \ldots \ldots \ldots \ldots \ldots \ldots \ldots \ldots \ldots \ldots \ldots$

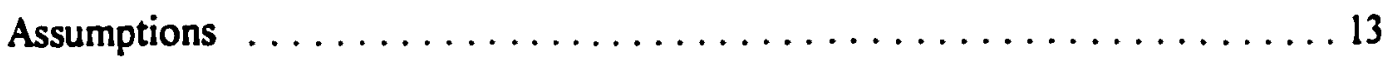

Chapter II. Critical Review of Relevant Literature

Psychoneuroimmunology: Linkage Between Stress and the Immune System . . . . 14

Stress-Related Immune System Changes in the Animal Model $\ldots \ldots \ldots \ldots \ldots$

Studies of Stress and the Immune System in Humans $\ldots \ldots \ldots \ldots \ldots \ldots \ldots$

Laboratory Induced or Scholastic Examination Stress . . . . . . . . . 21

Psychological Stress $\ldots \ldots \ldots \ldots \ldots \ldots \ldots \ldots \ldots \ldots \ldots \ldots$

Conditioned Immune Changes in Persons Undergoing Chemotherapy $\ldots \ldots \ldots 27$ 
Behavioral Interventions to Alter Immune Changes $\ldots \ldots \ldots \ldots \ldots \ldots \ldots . \ldots 29$

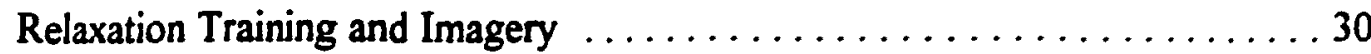

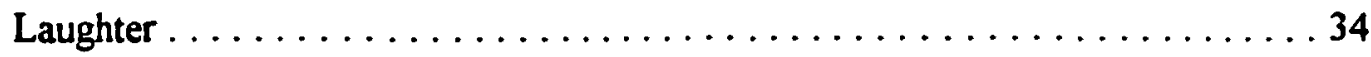

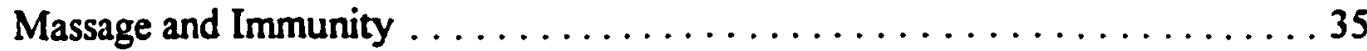

Structured Psychological Support and the Immune System . . . . . . . . 37

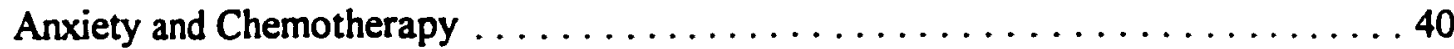

ANV as a Conditioned Response $\ldots \ldots \ldots \ldots \ldots \ldots \ldots \ldots \ldots \ldots \ldots \ldots \ldots$

Behavioral Studies to Modify Nausea and Vomiting $\ldots \ldots \ldots \ldots \ldots \ldots \ldots 44$

Behavioral Studies to Modify Anxiety ...................... 46

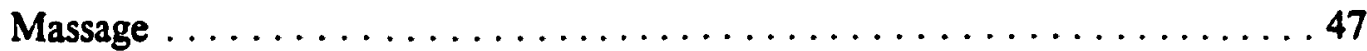

Progressive Muscle Relaxation $\ldots \ldots \ldots \ldots \ldots \ldots \ldots \ldots \ldots \ldots, 50$

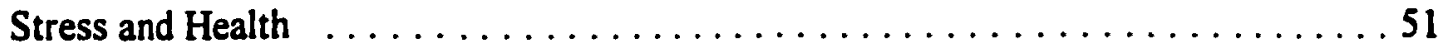

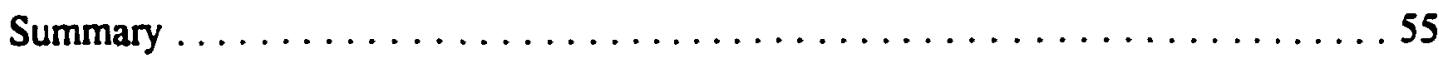

Chapter III. Methods and Procedures

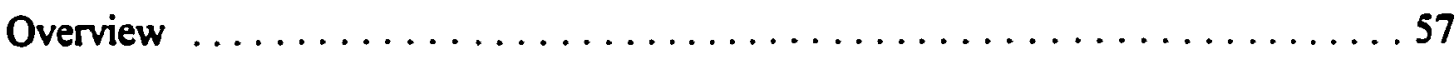

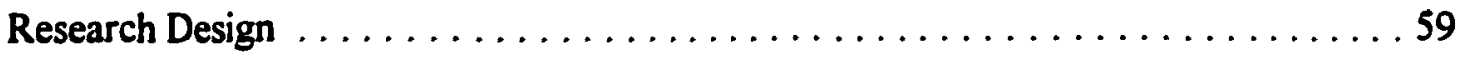

Sample Identification . . . . . . . . . . . . . . . . . . . . . . 59

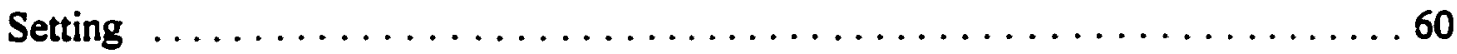

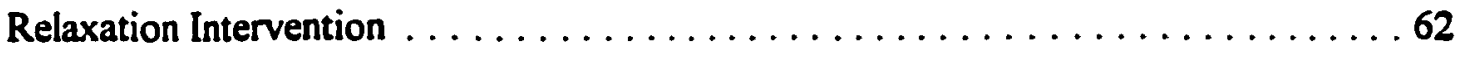

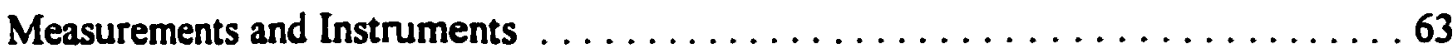

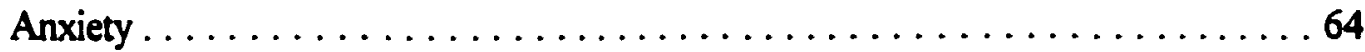

iv 


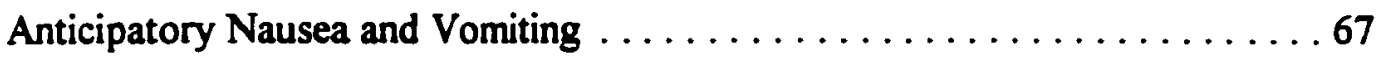

Immune Status $\ldots \ldots \ldots \ldots \ldots \ldots \ldots \ldots \ldots \ldots \ldots \ldots \ldots \ldots \ldots$

Procedure and Steps of Data Collection $\ldots \ldots \ldots \ldots \ldots \ldots \ldots \ldots \ldots \ldots \ldots \ldots$

Enrolling Subjects into Study $\ldots \ldots \ldots \ldots \ldots \ldots \ldots \ldots \ldots \ldots \ldots \ldots \ldots$

Baseline Assessment ........................... 76

Experimental Group $\ldots \ldots \ldots \ldots \ldots \ldots \ldots \ldots \ldots \ldots \ldots \ldots \ldots \ldots$

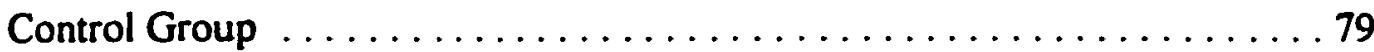

Completion of Participation $\ldots \ldots \ldots \ldots \ldots \ldots \ldots \ldots \ldots \ldots \ldots, 80$

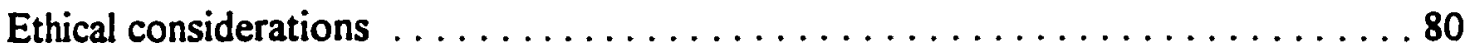

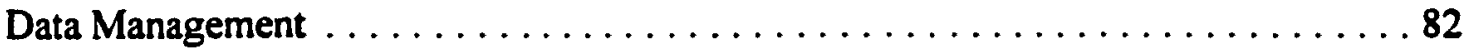

\section{Chapter IV. Findings}

Research Hypothesis and Goals: Results Summarized $\ldots \ldots \ldots \ldots \ldots \ldots \ldots . \ldots 84$

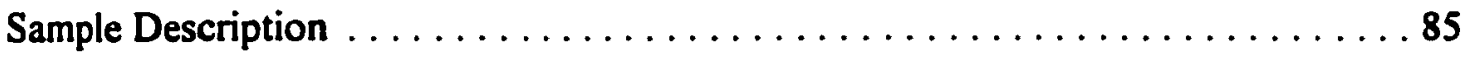

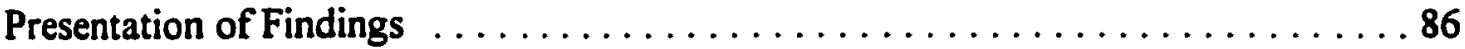

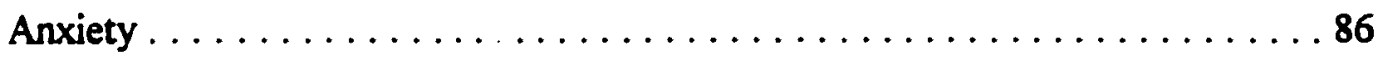

Anticipatory Nausea and Vomiting $\ldots \ldots \ldots \ldots \ldots \ldots \ldots \ldots . \ldots 9$

Immune Function $\ldots \ldots \ldots \ldots \ldots \ldots \ldots \ldots \ldots \ldots \ldots \ldots, \ldots \ldots \ldots$

Discussion of Findings $\ldots \ldots \ldots \ldots \ldots \ldots \ldots \ldots \ldots \ldots \ldots \ldots, \ldots$

Anxiety $\ldots \ldots \ldots \ldots \ldots \ldots \ldots \ldots \ldots \ldots \ldots \ldots \ldots \ldots \ldots, 98$

Nausea \& Emesis $\ldots \ldots \ldots \ldots \ldots \ldots \ldots \ldots \ldots \ldots \ldots \ldots \ldots . \ldots \ldots$

Immune Function $\ldots \ldots \ldots \ldots \ldots \ldots \ldots \ldots \ldots \ldots \ldots \ldots \ldots \ldots \ldots$

Proposed Plan for Further Study . . . . . . . . . . . . . . . . . . . 109 
Chapter V. Summary, Conclusions, and Recommendations

Summary of Study Findings $\ldots \ldots \ldots \ldots \ldots \ldots \ldots \ldots \ldots \ldots \ldots \ldots \ldots$

Methodological Limitations $\ldots \ldots \ldots \ldots \ldots \ldots \ldots \ldots \ldots \ldots \ldots \ldots \ldots \ldots$

Recommendations for Nursing Practice $\ldots \ldots \ldots \ldots \ldots \ldots \ldots \ldots \ldots \ldots \ldots$

Recommendations for Nursing Education $\ldots \ldots \ldots \ldots \ldots \ldots \ldots \ldots \ldots \ldots$

Recommendations for Further Research $\ldots \ldots \ldots \ldots \ldots \ldots \ldots \ldots \ldots$

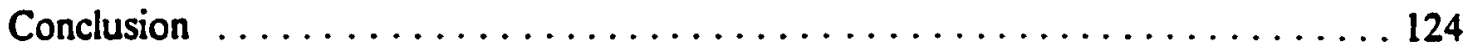

References $\ldots \ldots \ldots \ldots \ldots \ldots \ldots \ldots \ldots \ldots \ldots \ldots \ldots \ldots \ldots \ldots \ldots \ldots \ldots \ldots \ldots$

List of Appendices

Appendix A: PMR Audiotape Script $\ldots \ldots \ldots \ldots \ldots \ldots \ldots \ldots \ldots \ldots \ldots$

Appendix B: Approval from University of San Diego Committee on the

Protection of Human Subjects . . . . . . . . . . . 151

Appendix C: Approval from Loma Linda University Internal Review Board $\ldots 152$

Appendix D: Informed Consent Form $\ldots \ldots \ldots \ldots \ldots \ldots \ldots \ldots \ldots$

Appendix E: California Experimental Subjects' Bill of Rights . . . . . . 157

Appendix F: Demographic Profile and Data Collection Sheet $\ldots \ldots \ldots \ldots \ldots$

Appendix G: Descriptive Statistics for Immune Function Tests $\ldots \ldots \ldots \ldots$

List of Tables

Table I: Descriptive Analysis of Anxiety Measures $\ldots \ldots \ldots \ldots \ldots \ldots$

Table 2: Analysis of Covariance for Anxiety Measures $\ldots \ldots \ldots \ldots \ldots$

Table 3: Number of Subjects Who Reported Nausea and/or Emesis

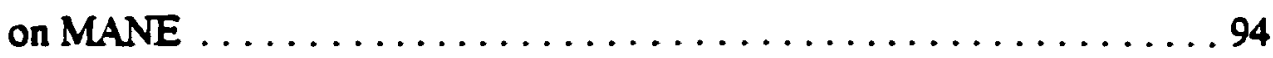

vi 


\section{List of Figures}

Figure 1: Study Model of Stress-Coping-Adaptation (SCA) Conceptual Framework $\ldots \ldots \ldots \ldots \ldots \ldots \ldots \ldots \ldots \ldots \ldots$

Figure 2: Study SCA Conceptual Framework with Relaxation Intervention . . . . 10

Figure 3: Overview of Methodology $\ldots \ldots \ldots \ldots \ldots \ldots \ldots \ldots \ldots \ldots \ldots$

Figure 4: State Anxiety Scores at Three Different Intervals According to Group and Amount of PMR Practice . . . . . . . 90

Figure 5: Trait Anxiety Scores at Three Different Intervals According to Group and Amount of PMR Practice .........92

Figure 6: Norepinephrine Levels According to PMR at Three Different Intervals According to Group and Amount of PMR Practice ......93 


\section{Chapter I}

Introduction

The immune system is an important protective mechanism against infection and some types of cancer. Studies of the immune system have revealed that physiological and/or psychological stress can modify immune function, in some situations resulting in immunosuppression. Although the association is not yet clear, alterations in immune function indicating immunosuppression are hypothesized to result in impaired host defense mechanisms.

Chemotherapy, a common treatment for cancer, is accompanied by side effects, such as immunosuppression and nausea and vomiting, that may affect the outcome of the therapy and patient compliance with the treatment regimen. Persons undergoing chemotherapy are not only stressed physiologically as a direct effect of the cytotoxic drugs, but also psychologically due to the diagnosis of cancer and anticipated side effects. Because of both physical and psychological side effects, chemotherapy may be considered a severe stressor (Razavi et al., 1993; Walker et al., 1999).

Because stress can down-regulate the immune system, it has been hypothesized that interventions that decrease the stress response may modulate the effects of stress. Behavioral strategies to promote relaxation have been studied and, in many cases, have shown promising results in lessening the degree of down-regulation in immune functions seen in persons subjected to stress, as compared with controls.

\section{Purpose}

The purpose of this pilot study was to examine the effect of a nursing relaxation intervention involving slow stroke back massage and progressive muscle relaxation on the neuro- 
immune response of chemotherapy recipients. Specifically, the study was designed to determine the effect of a nursing relaxation intervention on the extent of anxiety, immunosuppression, and anticipatory nausea and vomiting in chemotherapy recipients.

The study purpose was accomplished by using a prospective repeated measures design with an intervention and a control group. Baseline neuro-immune information was obtained on persons slated to undergo chemotherapy. A control group underwent routine chemotherapy with four cycles of Adriamycin and Cytoxan. An experimental group also underwent routine chemotherapy with four cycles of Adriamycin and Cytoxan, but in addition were exposed to a nursing relaxation intervention. After two cycles of chemotherapy had been completed, and just prior to the third dose, all subjects underwent a repeated neuro-immune assessment and assessment of chemotherapy-associated nausea and vomiting. All subjects were assessed again just prior to the fourth and final cycle of chemotherapy. Differences between the baseline and repeated measurements were evaluated, as were differences between the experimental and control groups.

\section{Background and Significance}

Recent research has demonstrated a predictable neuro-immune response to stress, resulting in depression of certain components of the immune system (Benschop et al., 1993; Blalock, 1994; Kropiunigg, 1993; Schedlowski et al., 1993). Responses to stress vary, depending on the nature of the stressor (Ader, Felten, \& Cohen, 1990), and can be summarized as follows: once sensory recognition of a stressor occurs, the body responds by central nervous system stimulation of the hypothalamus, inducing secretion of corticotropin-releasing hormone (CRH). 
$\mathrm{CRH}$ triggers release of corticotropin from the pituitary gland, which in turn stimulates the adrenal gland to produce glucocorticoids that maintain a feedback property of inhibiting $\mathrm{CRH}$ and corticotropin release. Glucocorticoids also can modulate immune function and metabolism (Blalock, 1994). Until recently, researchers have held a common understanding that while interplay between the neuroendocrine and psychological systems existed, the immune system functioned independently. Increasing evidence now supports a bidirectional modulation between immune and neuro-endocrine systems (Bellinger, Madden, Felten, \& Felten, 1994; Blalock, 1989). A meta-analysis of stress and immunity in humans has shown substantial evidence for a correlation between stress and immune system modulation and cellular decreases (Herbert \& Cohen, 1993a).

Studies of the immunological effects of stress have demonstrated a propensity for the development of conditioned responses (Ader \& Cohen, 1991; Ader et al., 1990). Ader and Cohen (1975) found that following illness-induced taste aversion conditioning using a saccharine drink paired with an immunosuppressant, rats exposed to saccharine drink alone demonstrated immunosuppression. Subsequent animal studies corroborated the fact that a conditioned stimulus can induce immunosuppression (Bovbjerg, Cohen, \& Ader, 1987; Kelley, Dantzer, Mormede, Salmon, \& Aynaud, 1985; Kusnecov, Husband, \& King, 1990; Lysle, Cunnick, Fowler, \& Rabin, 1988; Roudebush \& Bryant, 1991). Human investigations have indicated that some individuals undergoing chemotherapy develop anticipatory immunosuppression (Bovbjerg et al., 1990; Fredrikson, Furst, Lekander, Rotstein, \& Blomgren, 1993). While anticipatory immunosuppression has received little study to date, another induced effect, anticipatory nausea and vomiting (ANV) has been widely reported in the literature and may serve as a model for study 
of conditioned immunosuppression. An estimated $25-65 \%$ of patients undergoing chemotherapy experience ANV as a conditioned response to chemotherapy (Andrykowski, 1990; Andrykowski et al.,1988; Andrykowski \& Redd, 1987; Burish \& Carey, 1986; Morrow, 1992; Nicholas \& Hollandsworth, 1986). Anxiety has been found to be associated with ANV (Jacobsen, Bovbjerg, \& Redd, 1993; Jacobsen \& Redd, 1988; Lev, 1992). Studies involving a number of behavioral interventions have been reported to alleviate both anticipatory and treatment-induced nausea and vomiting. Behavioral interventions that have been found effective in diminishing anxiety, nausea, and vomiting associated with chemotherapy include progressive muscle relaxation training (PMRT) (Cotanch \& Strom, 1987), PMRT and guided imagery (Burish, Carey, Krozely, \& Greco, 1987), systematic desensitization (Morrow \& Morrell, 1982; Morrow et al., 1992), cognitive distraction and relaxation training (Vasterling, Jenkins, Tope, \& Burish, 1993), hypnosis (Jacobsen \& Redd, 1988), relaxation training and guided imagery (Lyles, Burish, Krozely, \& Oldham, 1982), and relaxation using combined slow-stroke massage and guided imagery (Scott, Donahue, Mastrovito, \& Hakes, 1986).

Immunosuppression is a common side effect of chemotherapy and may occur as a direct result of drug cytotoxicity (Fallowfield, 1992). In addition, anxiety is a common emotion experienced by cancer patients (Jacobson et al., 1993; Lev, 1992; Walker et al., 1999). Research indicates a correlation between anxiety level and incidence of ANV (Morrow, 1992) and between anxiety and anticipatory immunosuppression (Fredrikson et al., 1993).

Although many studies have identified the occurrence of immunosuppression, few studies have focused on interventions to modify stress-related neuro-immune changes (Sabbioni, 1993). 
Many behavioral interventions have been implemented and studied to help cancer patients deal with psychological reactions to cancer, but behavioral interventions as a means to modulate the immune system have received little study (Fawzy, Fawzy, \& Hyun, 1994; Schedlowski, Twews, \& Schmoll, 1994).

Certain interventions in healthy hosts without a specific identified stressor have been studied. Green and Green (1987), assessing the effects of relaxation on the immune system, found that subjects undergoing a relaxation response, massage, or visualization exhibited higher salivary immunoglobulin A (IgA) levels than did a control group in which touch alone was used. Groer et al. (1994) studied the effects of nursing back-rub on well, older subjects and found that salivary IgA levels increased for the experimental group.

Studies of the effects of mirthful laughter on the immune and neuroendocrine systems indicated an association between laughter and an increase in natural killer (NK) cell activity (Berk, Tan, Napier, \& Eby, 1989), an increase in spontaneous lymphocyte blastogenesis (Berk et al., 1988), and a decrease in stress-associated hormones (Berk, Tan, Fry, et al., 1989). Dillon, Minchoff and Baker (1985-86) found that salivary IgA concentrations increased significantly in subjects after they had viewed a humorous videotape, but not after viewing a didactic videotape.

Other studies have involved persons with an identified stressor. For example, a pilot study assessing relaxation training and immunological status of persons suffering bereavement suggested that relaxation training resulted in a promising psycho-immunological trend, with further study indicated (Houldin, McCorkle, \& Lowery, 1993). The authors did not describe the the trend noted. Kiecolt-Glaser et al. (1986) assessed intervention techniques with healthy 
subjects anticipating a stressor, measuring the effect of relaxation practice on cellular immunity in medical students undergoing final course examinations. While all subjects showed decreases in immunological response prior to the examination in comparison with the baseline, those who practiced the relaxation techniques had less decrease in helper/inducer cell percentages than did the controls, leading the researchers to suggest that intervention may be a factor in enhancing immunity during stressful events.

Literature review has revealed that while there are studies addressing anticipatory neuroimmune response before a stressor and neuro-immune responses following a stressor, there are few studies addressing whether intervention can modify the neuro-immune response. Prior to 1999 no study was found addressing the whole continuum, including assessment of neuro-immune status prior to onset of a stressor, with intervention to modify stress-associated response and assessment afterward to see if immune modification occurred as a result of the intervention.

There is a need for research regarding how specific stress characteristics may affect immune status. Research is also needed on the effectiveness of interventions to modify stress responses. If stressors that modify the immune system and result in immunosuppression are identified, then interventions could be planned to modify an anticipated negative stress response and prevent immunosuppression from occurring, or, potentially, to enhance immune function. No studies have been reported on the effects of bio-behavioral interventions in modifying the degree of anxiety and immunosuppression associated with chemotherapy, a known psycho-biological stressor.

Persons undergoing chemotherapy need to be studied prospectively to assess whether an intervention could enhance or protect their immune status. A decade ago, Kiecolt-Glaser \& Glaser 
(1988) recognized a need for prospective studies on the immunological effects of stress, but a review of literature published since then revealed that prospective studies, especially dealing with interventions, are not common. Relaxation strategies are a common part of nursing intervention; research on the efficacy of these interventions on the neuro-immune system could add greatly to the discipline, justifying the use of specific interventions in client care.

\section{Conceptual Framework}

The conceptual framework used for this study was the "Stress-Coping-Adaptation" (SCA) framework described by Monsen, Floyd, and Brookman (1992). The authors described the SCA process as "... the synthesis of the separate concepts of stress, coping, and adaptation into an interacting triad encompassing the reciprocal and continuous interrelationship of person/ environment in health states" (pp. 27-28). "The conceptualization of SCA succinctly expresses the person/environment health-related transaction and accounts for phenomena of concern in the development of science in nursing" (p. 28). In this model, the authors use Selye and Lazarus's concept of stress as a psychophysiological response of arousal in response to demands that are perceived as stressful in person/environment transactions. Stress has both a cognitive and a physiological component. Those stresses perceived as undesirable can result in health changes, and conversely, changes in health state can result in stress. Coping, considered from the context of Folkman and Lazarus, is how the individual manages perceived stressful demands of the person/environment relationship. A significant part of this model is the concept that nursing actions are seen as facilitating coping efforts through supportive or interventive care. Adaptation is considered the consequence of efforts to cope with the stress experience. It involves use of 
behaviors or cognitive measures to "... neutralize the stress experience and reestablish integrity of function" (p. 28). Effective adaptation requires a balance between stress and coping. If this balance is not achieved adaptation is not optimal, and the stress response continues.

For the purpose of this research, the SCA framework is conceptualized as the model displayed in Figure 1. Within the SCA conceptual framework stress is psychophysiological arousal that arises as a result of person or environmental transactions perceived as stressful by the individual. Coping is how the individual manages the perceived stress and involves both cognitive

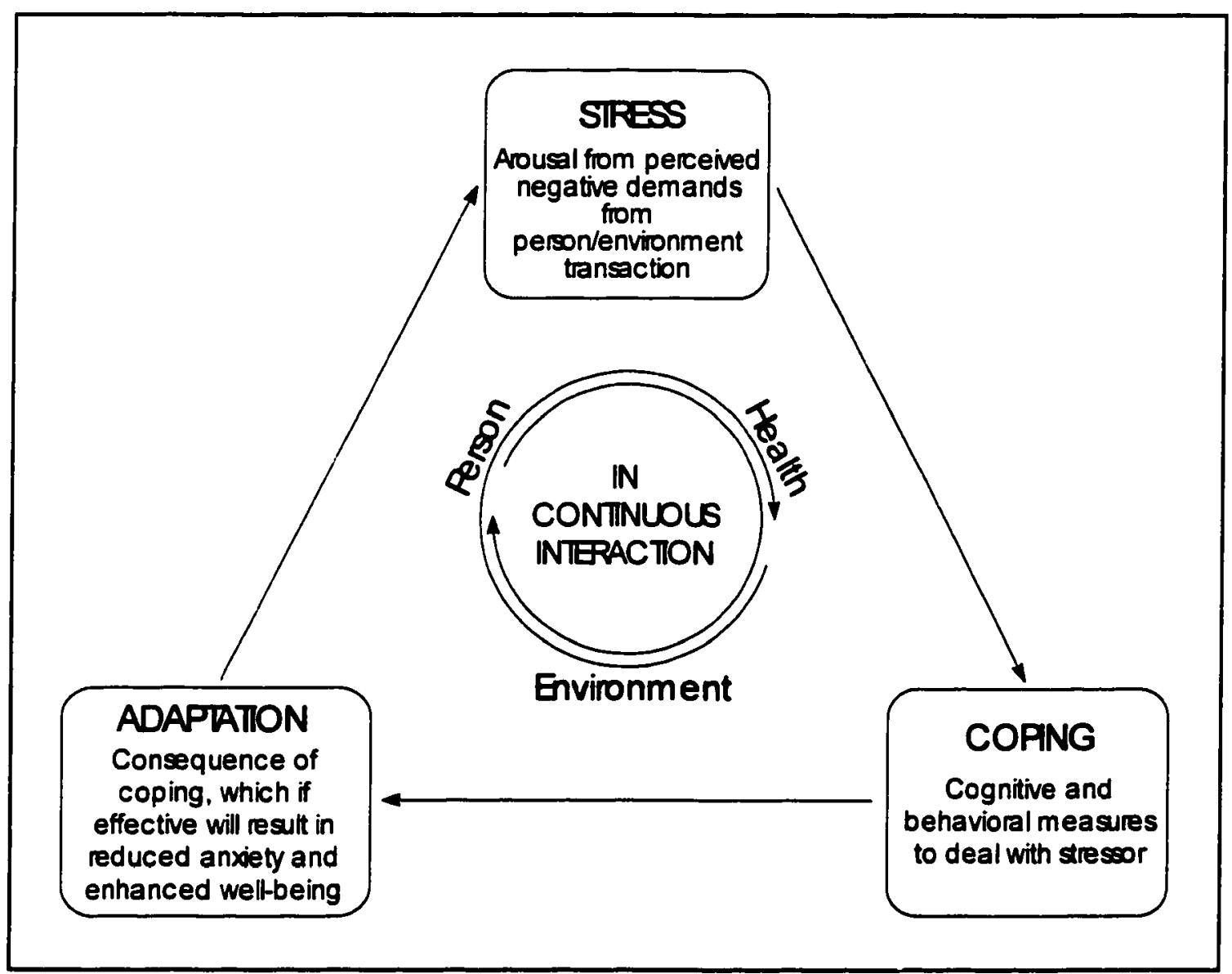

Figure 1. Study model of Stress-Coping-Adaptation (SCA) conceptual framework. 
and behavioral measures. Adaptation is the consequence of coping with the perceived stress. If coping has been effective the adaptation will result in reduced anxiety and enhanced well-being.

In this study stress was defined as a perceived negative experience, specifically that of chemotherapy with its associated physiological and psychological side effects, that resulted in a psycho-physiological response. Coping was the individual's response to deal with the perceived stress. A nursing relaxation intervention was incorporated to assist coping. The resulting adaptation was studied with physiologic assessment of neuro-immune functions, physiologic and psychological assessment of anxiety, and self-report of nausea and/or relaxation intervention.

Inclusion of the relaxation intervention into the proposed SCA conceptual model, with possible resulting altered stress response, is depicted in Figure 2. The stressor was chemotherapy, with the associated stress of the diagnosis of cancer and knowledge of potential side effects from therapy. The relaxation intervention targeted different parts of the model. The massage, given just before chemotherapy, was an attempt to diminish conditioned responses such as immunosuppression and anxiety associated with the chemotherapy administration, and to induce a relaxation response instead. Daily progressive muscle relaxation (PMR) practice was intended to enhance the coping response once the stressor had occurred and help the coping result in optimal adaptation. It was expected that positive adaptation would result in lower stress levels and a positive modulation in immune parameters in the intervention group versus the control group. Coping was viewed as a process to accomplish adaptation and was not the focus of this study. 


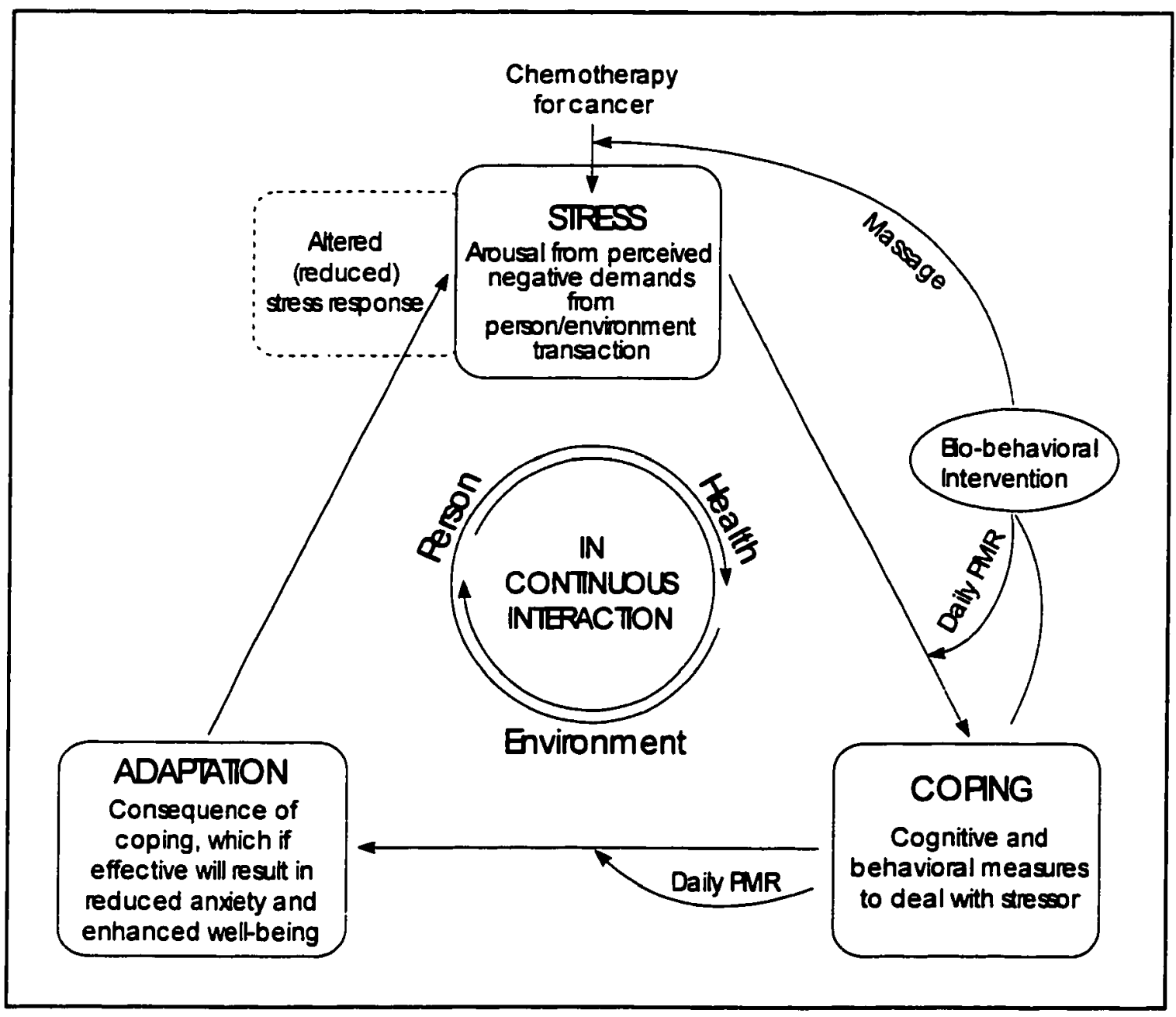

Figure 2. Study SCA conceptual framework, with relaxation intervention.

\section{Research Hypotheses}

The following research hypotheses were studied:

1. Relaxation intervention, in the form of slow-stroke back massage and progressive muscle relaxation, would reduce the degree of anxiety in clients receiving chemotherapy, as compared to those who did not experience the relaxation intervention. 
2. Relaxation intervention, in the form of slow-stroke back massage and progressive muscle relaxation, would reduce the degree of anticipatory nausea and vomiting (ANV) in clients receiving chemotherapy, as compared to those who did not experience the relaxation intervention.

3. Relaxation intervention, in the form of slow-stroke back massage and progressive muscle relaxation, would reduce the degree of immunosuppression in clients receiving chemotherapy, as compared to those who did not experience the relaxation intervention.

Because of the lack of information on bio-behavioral intervention and chemotherapy, additional goals of this pilot study were to expand on the available information on the effect of Adriamycin/Cytoxan (AC) chemotherapy on the immune system and to develop a plan for a further intervention study of persons undergoing chemotherapy.

\section{Definition of Major Variables and Concepts}

\section{Dependent Variables:}

Three dependent variables, related to persons undergoing chemotherapy, were included: anxiety, immunosuppression, and anticipatory nausea and vomiting.

Anxiety variables included state anxiety, which was defined as the anxiety level at the tested moment, and trait anxiety, referring to the subject's baseline level of anxiety. These were assessed using Spielberger's (1983) State Trait Anxiety Inventory (STAI-Y). Physiologic indicators of increased anxiety included blood pressure and heart rate, and the serum 
catecholamines epinephrine and norepinephrine, which have the potential for modulating the immune system (Ader, Cohen, \& Felten, 1995; Fredrikson et al., 1993).

Immunosuppression was defined as specified immune levels that show a significant drop from baseline level or lower than expected normal ranges based on standardized laboratory values. This was assessed by blood or serum analysis of Total Complement, Serum Immunoglobulin, Flow Cytometry Phenotyping of CD45-F/CD12-PE lymphocyte purity, CD3+ T-Lymphocytes (percent and absolute numbers), CD20+ B-Lymphocytes (percent and absolute numbers), CD3+25+ IL-2 Activated T Lymphocytes (percent and absolute numbers), CD3CD16+56+ NK Cells (percent and absolute numbers), Mitogen Stimulation Assay with PHA, Natural Killer Cell Assay (Effectors, Target Cells, 40:1, 20:1, 10:1, 5:1 and Lytic units), and Cytokines $\mathbb{L}-2, \mathrm{IL}-4, \mathrm{IL}-6, \mathbb{L}-10, \mathbb{I}-12$, and INFy.

Anticipatory nausea and vomiting (ANV) was defined as the nausea or vomiting that occurred before or during chemotherapy in anticipation of the treatment (Burish \& Carey, 1986; Morrow, 1992). ANV was assessed using the Morrow Assessment of Nausea and Emesis (MANE) instrument.

\section{Independent Variable}

The independent variable was the relaxation intervention planned to elicit a relaxation response in subjects. The relaxation intervention consisted of a 10-minute slow-stroke back massage just before administration of chemotherapy and daily practice of progressive muscle relaxation. For this study, researcher interaction was considered a part of the relaxation intervention because the researcher administered the massage and spent time with the 
experimental subjects during unavoidable waiting periods and blood specimen draws. In addition, the researcher's voice was on the guided progressive muscle relaxation tape.

\section{Attribute Variables}

Selected attribute variables were recorded for each subject, including birth date, cancer diagnosis (including extent, cellular type, and intervention), any medical problems, and associated medications. Cardiovascular assessment, including heart rate and blood pressure, was recorded on experimental subjects during the relaxation intervention. These were to be obtained from clinic records for all subjects during administration of chemotherapy, but were not consistently recorded.

\section{Assumptions}

The research was based on the following assumptions: (1) patients undergoing chemotherapy would be able to distinguish between the often subjective sensations of nausea and anxiety when self-reporting on the STAI-Y and the MANE; (2) laboratory tests would provide accurate measurement of immune function and serum catecholamine levels; (3) specimens would be handled and reported accurately by the laboratories; (4) if immunological changes occurred, they would be measurable; (5) clinic equipment used and maintained according to protocol would assess cardiovascular parameters of heart rate and blood pressure accurately; (6) outpatient clinic staff would cooperate with the study and consistently observe clinic procedures; (7) relaxation strategies would actually result in relaxation; and (8) experimental subjects would practice the relaxation exercises as requested. 


\section{Chapter II}

Critical Review of Relevant Literature

A brief introduction and overview of psychoneuroimmunology (PNI), showing linkages between stress and the immune system will be provided as a background to the immunological studies that will be reviewed. This will be followed by animal and human PNI studies of the immune system, with a focus on conditioned changes. Based on the hypothesis by Green \& Green (1987) that if stress contributes to immunosuppression, relaxation may enhance the immune system, studies reflecting interventions to modify immune responses are reviewed. Finally, the phenomenon of conditioned anticipatory nausea and vomiting is reviewed.

\section{Psychoneuroimmunology: Linkage Between Stress and the Immune System}

Human response to stress has been extensively studied and reported in the health-related literature. Responses to stress vary, depending on the nature of the stressor (Ader et al., 1990). According to Weizman and Bessler (1999), "The correlation between stress and immunity is not linear, and both increases and decreases in immunocompetence can occur following exposure to different stressors" (p. 9).

The body's response to stress can be summarized as follows: once there is sensory recognition of a stressor, the body responds by central nervous system stimulation of the hypothalamus, causing secretion of corticotropin-releasing hormone (CRH). CRH stimulates release of corticotropin from the pituitary, which in turn stimulates the adrenal gland to produce glucocorticoids. Glucocorticoids not only have a feedback property of inhibiting CRH and corticotropin release, but also can modify immune function and metabolism (Blalock, 1994). 
There is also a direct bidirectional connection between the central nervous system (CNS) and the immune system through innervation of lymphoid tissues. These nerve fibers connect at neuroeffector junctions with lymphocytes and macrophages. Neurotransmitters, released from the nerve fibers, can diffuse to act at distant sites and also can act on immune cells such as lymphocytes, granulocytes, and monocytes that have neurotransmitter receptors, and cytokines released by immune cells can influence CNS activity (Ader et al., 1995).

Recent research has demonstrated evidence for interaction between the immune system and the neuroendocrine system, with the immune system capable of regulating neuroendocrine function and vice versa (Ader et al., 1995; Bellinger et al., 1994; Blalock, 1989). This has given rise to research in the field of psychoneuroimmunology, which studies the mind-body interaction from a holistic paradigm or, as defined by Nguyen (1991), as the " ... field of research that investigates how the central nervous system (CNS) and the immune system may interact to influence health" (p. 1). The focus of this study is on the neuro-immune response to a chemotherapy stressor that involves both physiologic and psychological stress for the recipient.

Review of relevant literature revealed that there is much written about the effects of stress in general on the emotional and physical health of individuals. Adler (1994) suggested that persons undergoing stress may experience increased susceptibility to disease, including cardiovascular and infectious diseases and complications during pregnancy. Rice (1992) and Sparacino (1982) associated adverse emotional and mental health with stressful situations. Benschop et al. (1993, p. 1825), reporting on the relationship of psychobiological factors and immune functions, stated that "... inadequate coping with life stress, as reflected by high levels of 
self-reported symptoms, affects immune function." Some interesting animal research raises a question of what effect stress has on cancer metastasis and response to treatment. Mice with Lewis lung tumors were found to have significant increase in tumor metastasis when stressed, as compared with non-stressed mice (Giraldi, Perissin, Zorzet, \& Rapozzi, 1992). Later research by members of this team found that stress negated the antitumor effect of cyclophosphamide in treated mice with Lewis lung tumor, in comparison with non-stressed mice (Zorzet, Perissin, Rapozzi, \& Giraldi, 1998). While the mechanism of the relationship between health and stress-induced immunosuppression may not be clear, there is sufficient information to suggest that there is a negative association.

The endocrine system responds to stress with a variety of stress hormones (Blalock, 1994; Frankenhauser, 1971; Henry, 1987; Kim, 1987; Lundberg, 1976; Siconolfi, 1994), with the response to perceived stress occurring quickly. Epinephrine and norepinephrine are secreted within 2 to 3 minutes of perception of a stressor, and the effects of sympathetic stimulation are maintained for 1 to 2 hours (Robinson, 1990). Catecholamines have an effect on the immune system. The CNS and the immune system are linked both directly through neurological innervation of immune system tissues, and indirectly through soluble neuro-endocrine or biochemical products (Ader et al., 1995; Zeller, McCaine, \& Swanson, 1996).

Research on the effects of stress on the immune system has indicated that there is a neuroimmune response to stress, resulting in a depression of certain aspects of the immune system (Ader \& Cohen, 1993; Benschop et al., 1993; Kropiunigg, 1993; Schedlowski et al., 1993; Walker, Johnson, Whiting, Heys, \& Ermin, 1993). Stress, social disruption, and depression are 
associated with activation of the hypothalamic-pituitary-adrenocortical (HPAC) axis, with resulting secretion of catecholamines and other substances. Increase in catecholamines has been associated with reduction in peripheral lymphocyte numbers, reduced phytohemagglutinin (PHA) response, and suppression of natural killer (NK) cell activity (Khansari, Murgo, \& Faith, 1990; Kiecolt-Glaser, Cacioppo, Malarkey, \& Glaser, 1992; O'Leary \& Miller, 1991; Schedlowski et al., 1993). There is evidence to support the correlation of increased stress hormones with depression of certain aspects of the immune system (Ader et al., 1995; Ader \& Cohen, 1991; Ader et al., 1990; Cupps \& Fauci, 1982; Landmann et al., 1984; Linn, Linn, \& Jensen, 1981; Zeller et al., 1996 ). A meta-analysis of stress and immunity in humans has shown substantial evidence for an inverse correlation of stress with immune function and cellular decreases (Herbert \& Cohen, 1993a).

According to Kiecolt-Glaser et al. (1992), immune system changes that follow short-term stress mimic changes that occur following injection of epinephrine and seem to "...reflect transient alterations in lymphocyte migration from lymphoid organs and peripheral blood mediated through adrenergic receptors on lymphocytes or via the sympathetic innervation of lymphoid organs" (p. 680). These changes include increased NK cell percentage, decreased blastogenic response to mitogens, and decreased CD4 cell percentages, while monocytes and total T cells remain unchanged. Benschop et al. (1993) also reported that NK cells are sensitive to changes under stressful situations, although a study by the authors could not show an association between stressful situations and decreased NK cell activity. First-time parachutists, undergoing what can be considered the acute psychological stressor of a first jump, were assessed for sympathetic adrenal 
hormones and immune changes prior to, during (with use of a small portable pump attached to an intravenous catheter), and following the jump. As expected, adrenalin and noradrenaline levels increased during and immediately following the jump. There was an increase in NK cell activity and an increase in lympinocyte subsets immediately following the jump. However, an hour later there was a significant decrease below starting values. This study indicated that short-term psychological stress resulted in increased secretion of sympathetic adrenal hormones and in changes in NK cell activity and numbers, supporting the theory of nervous and immune systems interacting during stress (Schedlowski et al., 1993). One may also conclude that the time interval after the stress may be important when assessing certain immune functions.

\section{Stress Related Immune System Changes in the Animal Model}

Studies on the immunologic effects of stress have shown that these effects can be conditioned (Ader \& Cohen, 1991; Ader et al., 1990). An early study by Ader and Cohen (1975) found that following illness-induced taste aversion conditioning, using a saccharine drink paired with an immunosuppressant, rats exposed to the saccharine drink demonstrated immunosuppression. Subsequent animal studies have also demonstrated that a conditioned stimulus can result in immunosuppression (Bovbjerg et al., 1987; Kelley et al., 1985; Kusnecov et al., 1990; Lysle et al., 1988; Roudebush \& Bryant, 1991). A review of selected studies follows.

Based on the idea that behavioral conditioning may result in immunosuppression, Ader and Cohen (1975) performed a study in which rats were conditioned by pairing a saccharine drink with cyclophosphamide injection. Non-conditioned animals were given water with saccharine and injected with cyclophosphamide, while the control animals were given plain water and 
distilled water injection. Animals given the saccharine and cyclophosphamide injections developed an aversion to the saccharine solution. Immune study revealed significant immunosuppression in animals that were previously conditioned and were given saccharine prior to or following antigen administration.

Grochowicz et al. (1991) used the same technique of taste aversion conditioning, combining oral saccharine and injection of cyclosporin ten and six days prior to heart allograft in rats. The conditioned rats were given saccharine alone one day before and three days after heart transplantation. The conditioned animals had significantly prolonged heart graft survival as compared to conditioned control groups, indicating that immunosuppression occurred as a conditioned response.

Roudebush and Bryant (1991) used the conditioning technique of pairing saccharine with cyclophosphamide injection to study a delayed type hypersensitivity response in mice. Experimental animals were first conditioned, and all animals sensitized with $5 \%$ picryl chloride (PCL). On the third day, 50 $\mu \mathrm{L}$ of PCL were applied to a hind footpad of all the mice. Conditioned animals were also given saccharine as drinking solution. Conditioned mice demonstrated significantly less delayed hypersensitivity type paw swelling than did unconditioned mice. Another interesting finding was that when all mice were sacrificed and studied, no significant differences in serum corticosterone levels were found in nonconditioned or in conditioned groups of mice, showing that the conditioned immunosuppression that occurred was not associated with increased glucocorticoid levels. 
Physical stressors such as electric shock have also been found to be immunosuppressive in rats (Lysle et al., 1988). Laudenslager, Ryan, Drugan, Hyson, and Maier (1983) found that rats who were exposed to inescapable shock demonstrated suppression of mitogen-stimulated lymphocyte proliferation (with phytohemagglutinin and concanavalin A), as compared to rats exposed to escapable shock. The authors concluded that not only did shock affect the immune response, but also that the aspect of controllability was important in immune system modulation.

Lysle et al. (1988), building on the theory that stresses such as electric shock can be immunosuppressive, studied whether a non-aversive stimulus of a psychological nature that had been associated with shock could also affect immune function. Rats were placed into groups that were conditioned with shock plus sound, shock plus light, or shock alone, and a control group that received handling and transporting but no shock. Results indicated that when the conditioned rats were exposed to the stimulus, they developed suppressed lymphocyte reactivity to mitogens, indicating immunosuppression. These results support the theory of occurrence of conditioned immunosuppression, and also that a psychological stressor can result in conditioned immunosuppression.

While most studies focused on conditioned immunosuppression, Bovbjerg et al. (1987) studied immune enhancement in a study of behavioral conditioning involving mice. Mice were first conditioned using saccharine and cyclophosphamide. They were subsequently given saccharine drink on three days following sensitization, and then injected with a low dose of cyclophosphamide. When then challenged with sheep red blood cells (SRBC), the mice initially demonstrated immunosuppression. However, when re-challenged with SRBC on days 11 and 18, the mice demonstrated significantly enhanced 
delayed type hypersensitivity compared to nonconditioned and placebo mice. Ader, Kelly, Moynihan, Grota, and Cohen (1993) were also able to demonstrate conditioned enhancement of a specific antibody titer in experimentally conditioned mice.

Of interest is the implication for behavior modification and the immune system. Miller, Thomas, Pacheco, and Rollwagen (1993) found that rats subjected to stress by electric shock that were able to avoid shock by pressing a lever had higher NK activity and mitogen-stimulated proliferation of their splenocytes than did control rats or rats that were not able to avoid shock, implying that control of the situation affected immune outcome. Laudenslager et al. (1983) suggested that control of stress is an important aspect of immune system modulation.

\section{Studies of Stress and the Immune System in Humans}

Persons experiencing naturally occurring stress or laboratory-induced stress have demonstrated significant immunological changes. Selected studies will be reported dealing with laboratory-induced or scholastic examination-induced stress, psychological stress, and chemotherapy-associated stress. This will be followed by studies of behavioral interventions to modify stress-related immune changes.

\section{Laboratory Induced or Scholastic Examination Stress}

The effect of examination stress on selected immune functions of healthy students has been studied. One study included 23 undergraduate psychology students. Students were placed in two groups, with an experimental group taking examinations at two time periods, and a control group for which no examination was required. Anxiety/tension was assessed by self-report and with the STAI. Blood was drawn from experimental subjects 3 times; 6 weeks before the first 
examination, 1 day before the examination, and 12 to 14 days after the examination. Control subjects underwent psychological assessment and had one blood sample drawn at the same time as experimental subjects' second blood draw. Blood was tested for monocyte distribution, expression of IL-2 receptor, and mitogen stimulation. Results indicated that under acute examination stress, there was an increase in the number of monocytes and a reduction of the CD4:CD8 fractions. There were also reductions of cells expressing the IL-2 receptor and in the proliferative responses in IL-2 enriched culture medium. The third sample, obtained, nearly two weeks after the examination, indicated a decrease in mitogen and antigen response. The findings suggested that under acute examination stress, a decrease of certain cellular immunological functions occurred (Halvorsen \& Vassend, 1987).

Another study of the effect of a naturally occurring stress on healthy people examined 75 medical students experiencing examination stress. Baseline blood samples were drawn one month prior to, and a final sample drawn on the day of final examinations. Results showed a significant decrease in NK cell activity from the baseline to the second sample. In addition, persons who scored high on scales of stressful life events and loneliness demonstrated lower levels of NK cell activity. While plasma IgG and IgM and salivary IgA did not change, plasma IgA showed an increase from the first to the second sample. Decreased NK activity indicated immunosuppression associated with the mild stressor of examination, as well as with stressful life events and loneliness (Kiecolt-Glaser, Garner, et al., 1984).

A very briefly reported study (Fittischen, Schultz, Schultz, Raedler, \& von-Kerekjarto, 1990) associated examination stress with a significant increase in the percentage of large 
immunocytes in the peripheral population. The significance of the findings was not discussed, except to imply that there was significance and that further study was warranted.

Bachen et al., (1992) studied the effect of acute laboratory-induced psychological stress on certain aspects of the immune system in 43 healthy young men, with 33 in the experimental and 10 in the control groups. All subjects initially underwent immune assay, including T-mitogenic responses and circulating lymphocyte populations. Control subjects relaxed, while experimental subjects underwent a frustrating laboratory situation, following which the immune assay was repeated. Stressed subjects showed increases in heart rate and in systolic and diastolic blood pressure from baseline, as compared to controls, who did not show the increases. Stressed subjects also demonstrated a significant decrease in T-cell mitogenesis from baseline to poststressor measures, while controls showed an increase, thought by the researchers to be likely because their task was to relax during the time that stressed subjects underwent their frustrating task. Stressed subjects also showed a decline in the CD4:CD8 ratio, with an increase in the CD8 cells and decrease in CD4 cells from baseline to post-stressor measurement, and an increase in circulating NK cells. These changes were not seen in controls, leading the authors to conclude that changes in the experimental subjects' immune function resulted from the acute mental stressor.

\section{Psychological Stress}

Depression, bereavement, and loneliness have also been associated with immunosuppression (Andreoli et al., 1993; Herbert \& Cohen, 1993b; Irwin, Daniels, Bloom, Smith, \& Weiner, 1987b; Kiecolt-Glaser, Rickers et al.,1984), as has high state anxiety in 
stressed individuals (Fredrikson, et al., 1993; Halvorsen \& Vassend, 1987; Linn et al., 1981). A longitudinal study of women with terminally ill spouses, that assessed them prior to and following the death of the spouse, and another study that assessed bereaved women who had lost a spouse to lung cancer within the previous one to four months showed that the bereaved women had a significant decrease in NK activity when compared to controls. Depressive symptoms, rather than the death of the spouse alone, were found to be associated with the immunosuppression (Irwin, Daniels, Bloom, Smith, \& Weiner, 1987a \& 1987b).

A study of non-psychotic psychiatric inpatients showed that patients who reported high loneliness levels exhibited higher levels of urinary cortisol and immune system suppression, demonstrated by significantly lower NK activity and less T-lymphocyte phytohemagglutinin response, than did persons reporting low loneliness (Kiecolt-Glaser, Rickers, et al., 1984).

The relationships among depression, age, and immunity were studied in a group of 53 untreated adults presenting to a psychiatric clinic with a major depressive episode (MDE). Fiftythree healthy matched controls were included in the study. At the initial screening, a comprehensive psychosocial and diagnostic assessment was made on each, using standardized instruments. Blood was collected for assessment of immune status, including cell counts, cell surface phenotyping, and mitogen stimulation. The only difference noted between the groups was a trend of decreased number of NK cells in the MDE group, as compared to the control group. However, in the MDE group, increasing age was significantly correlated with decreased numbers of total lymphocytes and decreased mitogen response, while this was not noted in controls, leading the authors to suggest that age and clinical characteristics of depression may be 
independent factors to consider when studying major depression and psychoneuroimmunology (Andreoli et al., 1993).

Depression has also been associated with a decrease in cellular immunity to VaricellaZoster virus (VZV). Irwin et al. (1998) evaluated a total of 53 subjects, examining the relationship between VZV-specific immunity and depression and age. The findings showed a marked decline in VZV-specific cellular immunity in the depressed group, as compared to the non-depressed group. In addition, an age-related decline in VZV-specific cellular immunity was found in non-depressed adults.

In a study of patients undergoing chemotherapy for breast cancer, Levy, Herberman, Lippman, and d'Angelo (1987) found that stressful factors, such as lack of social support, depression, or fatigue, correlated with depression of NK cell activity, whereas chemotherapy, an expected immunosuppressant, did not show the same correlation. The authors, expressing their surprise at this finding, noted that due to the measurement interval, their assessment did not evaluate short-term or acute treatment-related effects on NK cells. They suggested that NK cell recovery may have occurred during the intervals between doses.

Linn et al. (1981) studied the association between immune functioning and levels of anxiety in chronically ill patients who were undergoing the stressor of a hospitalization. Subjects were assessed at time of hospitalization and 75 were screened to be free of autoimmune disease or cancer and included in the study. Anxiety was assessed using the Anxiety Factor of the Hopkins Symptoms Checklist. Humoral immunity was assessed using immunoglobulins IgG, IgA, and IgM. Cellular immunity was assessed lising three skin tests, for in vivo testing, and three mitogens for in 
vitro testing. The researchers found that stressed patients exhibited decreased T-cell response to mitogens, suggesting an alteration in the in vitro assessment of cellular immunity. However these same patients showed more likelihood of multiple positive in vivo responses to skin tests. These mixed results have been found in other studies indicating that variables such as anxiety or stress may be immunosuppressive or immunoenhancing, depending on the tests being studied. The authors stated that the increased response to skin tests could indicate an increased susceptibility to disease. A study by Snyder, Roghmann, and Sigal (1993) examined the effects of stress and psychosocial factors on primary cellular immune responses in 89 healthy females. All subjects filled out questionnaires to assess stressful life events and psychosocial factors such as social support. Blood was drawn for baseline lymphocyte proliferation (LP) assessment, following which each subject was immunized with a subcutaneous injection of $100 \mu \mathrm{g}$ keyhole limpet hemocyanin $(\mathrm{KLH})$, a novel antigen. Repeat assessments occurred at three and eight weeks following the first visit. Findings revealed a trend of decreased LP associated with increased "bad stress" and significantly decreased LP associated with increased level of hassles at the three-week measurement when compared to the baseline measures. Subjects reporting "good stress" showed a trend of increased LP at the three-week measurement. At the eight-week measurement all subjects showed an enhanced LP effect. The conclusion drawn was that even minor life psychological stress can influence the immune system; the authors suggested that the findings may help explain individual responses to immunizations (Snyder et al., 1993).

Lymphocyte subpopulations and plasma catecholamine and cortisol levels in 15 normal subjects before and following physical and psychological stress were assessed by Landmann et al. 
(1984). Following physical stress, all circulating leukocyte subpopulations, but especially B and T cells, were increased, while following psychological stress, the numbers of circulating B cells, monocytes, and NK cells increased. The authors interpreted these cell distribution patterns following stress as suggestive of immunosuppression and thought them to be related to adrenalin production during stress.

A meta-analysis by Herbert and Cohen (1993b), studying the association between psychological depression and immunity, concluded that depression was significantly associated with decreased lymphocyte function (including mitogen stimulation with PHA, Con A, and pokeweed antigen), and decreased NK cell activity. Higher numbers of circulating white blood cells (neutrophils and monocytes) were also found, with decreased numbers of lymphocytes (NK, $\mathrm{B}$, and T-cells) and decreased ratio of helper to suppressor $\mathrm{T}$ cells.

\section{Conditioned Immune Changes in Persons Undergoing Chemotherapy}

Recent human studies have revealed that an anticipatory immunosuppression occurs in some individuals undergoing chemotherapy. Bovbjerg et al. (1990) studied 20 patients who were receiving chemotherapy for ovarian cancer. Patients were to receive chemotherapy in the hospital every four weeks. After the patients had received at least three chemotherapy infusions, they were entered into the study. In the four-week interval prior to the next scheduled infusion, subjects were contacted and arrangements made for assessment to be done in the home setting, three to eight days before the next planned hospitalization. Assessment included psychological and behavioral questioning, using the STAI questionnaire and the VAS rating of nausea and anxiety. A blood sample was obtained by venipuncture for immune assessment, including functional assays 
of NK cell activity and proliferative responses to mitogens, as well as quantitative assays, including complete blood cell counts and subset numbers. The second set of data was obtained in the hospital on the morning prior to chemotherapy infusion. Results showed that in the hospital setting, proliferative responses to mitogens were significantly lower than in the home setting. No significant differences were found in NK cell activity or in quantitative assay between home and hospital. These findings indicated that there was an anticipatory decrease in immune function, suspected to be a result of conditioning from repeated pairing of immunosuppressive chemotherapy and immunosuppression. Of interest is the finding that subjects showing anticipatory immunosuppression had higher levels of nausea in the hospital than those without anticipatory immunosuppression.

Fredrikson et al. (1993) conducted a second study of conditioned immunosuppression resulting from chemotherapy. The primary aim of this study was to see if patients undergoing a somewhat less aggressive chemotherapeutic regimen than that used in the 1990 Bovbjerg et al. study would also exhibit anticipatory immunosuppression. An additional point of the study was to examine whether anxiety was related to immunocompetence. The study included 27 patients with verified breast cancer who were to undergo chemotherapy with 6 courses of CMF (Cyclophosphamide, Methotrexate, and 5-Fluorouracil). Each course was repeated every 28 days. Twentyfive healthy hospital staff females were included as controls. Blood for immune function assessment was obtained in the patients' homes two days before and then again immediately before their fourth or fifth course of chemotherapy in the hospital outpatient chemotherapy department. Immunological assessment included identification of lymphocyte subsets (CD3, CD4, 
CD8, CD16, and CD20), spontaneous cytotoxicity of lymphocytes, and mitogenic responses of lymphocytes (using Concanavalin A). Similar samples were obtained on controls. In addition, psychological assessment using the trait portion of the STAI was performed by giving the questionnaire to subjects when the first blood sample was obtained, to be collected two days later with the second blood sample. Overall findings revealed that patients had an increase in total granulocytes $\left(10^{9} /\right.$ liter $)$ but a significant decrease in the percentage of monocytes and lymphocytes when in the out-patient hospital setting compared to the home setting. Patients with high anxiety demonstrated compromised NK cell activity and had reduced total numbers of monocytes, as compared to patients with low anxiety. Controls did not demonstrate differences between home and hospital. Studies have shown that release of adrenalin (epinephrine) can mobilize granulocytes to move from storage tissues into the circulation, resulting in an increase of circulating granulocytes. The increase in the total number of granulocytes in peripheral blood of patients was thought by the researchers to be related to stress and release of adrenalin. It was hypothesized that adrenalin-mediated changes, such as increase of granulocytes, may be followed later by other immunosuppressive changes due to catecholamines (Fredrikson et al., 1993).

\section{Behavioral Interventions to Alter Immune Status}

In 1993, Sabbioni noted that little study had been reported on interventions to modify stress-related neuroendocrine-immune changes. However, with the increasing interest in psychoneuroimmunology, there is greater interest in this area, and an increasing number of studies are being conducted and reported. Research studies that address selected behavioral methods to alter immunity are reviewed here. 


\section{Relaxation Training and Imagery}

Green, Green, and Santoro (1988) conducted a study in which 40 volunteers were randomly assigned to a waiting list control group, or to an experimental group in which the subjects practiced a relaxation technique daily for 3 weeks. The 24 subjects in the relaxation group were randomly assigned to practice 1 of the 3 following relaxation techniques: a relaxation response while sitting up, a relaxation response while lying down, or guided visualization while lying down. Experimental subjects were directed to practice the relaxation technique daily for 20 minutes while at home. They also received relaxation training on a weekly basis for three sessions. Blood and saliva samples were obtained prior to and immediately following relaxation practice on the experimental group at three intervals: the first day of the study, at day twenty-two after three weeks of practice, and, for the eleven subjects who remained in the study, at day forty-three. Blood and saliva samples were obtained on the waiting list control group at three intervals; before and after relaxation training on day one, and three weeks following start of the study. Findings showed a significant increase from baseline in salivary IgA and in serum IgA, IgG, and IgM in subjects who practiced relaxation daily over a three-week period regardless of technique used, leading the authors to suggest that relaxation could be a coping strategy that may positively affect some aspects of the immune system.

A pilot study by Houldin et al. (1993) assessed relaxation training and immunological status of persons suffering bereavement. Nine females whose spouses had recently died of cancer were recruited for the study. Each underwent a one-hour relaxation training program involving progressive muscle relaxation training and guided imagery on a weekly basis for four weeks. A 
standardized panel of psychological and immunological tests was used for assessment. Specific findings of the study were not reported, but findings were summarized to imply that a selective decrease in immune function, specifically IgG, IgA, and total lymphocytes was associated with increased levels of psychological distress. In addition, a higher infection rate with herpes simplex type I antibody (70\%) was reported in the research group compared with the normal population (50\%), but after relaxation training there was a decrease in the rate of infection (specific data were not given). The authors do note that there are limitations to the study, including the small sample size and lack of an experimental design, but suggest that relaxation showed a positive impact on immune function and the decreased rate of infection finding was a basis for further study.

Kiecolt-Glaser et al. (1986) assessed whether cellular immunity could be modulated in 34 healthy medical student volunteers. Immune assessment occurred one month after one set of academic examinations, one month prior to, and the day of the next set of examinations. Psychosocial assessment was performed, using self-report questionnaires. Half of the subjects were assigned to a control group that was offered the relaxation training the following month. The other half of the subjects were assigned to a professionally-led hypnosis/relaxation group that began with standard deepening exercises, followed by self-hypnosis, progressive relaxation, imagery exercise, and/or autogenic training. The end of the session included suggestions for relaxation during the day and enhanced study success. Students were encouraged to relax during the week. Immune assessment revealed that the percentage of helper/inducer cells and the helper/suppressor cell ratio showed significant decrease in all subjects for the examination sample 
compared to the baseline measurement. Overall, both control and experimental subjects showed lower than expected values even in the baseline samples, leading the authors to conclude that the low baseline values may be stress-related. After correcting for differences in baseline levels, an association was seen between frequent relaxation practice and increased helper/inducer percentages, but not with NK activity. The authors concluded that relaxation practice may be associated with enhancement of some aspects of cellular immune functions.

Zachariae et al. (1990) studied the effect of relaxation and guided imagery on cellular immune function in 10 healthy subjects. The aim of the study was to examine the effects of a positive psychological experience on the immune systems of healthy volunteers. A baseline blood sample was obtained on the first day of the study, after which each subject underwent a 45-minute session of guided imagery and relaxation; a second blood sample was then obtained. Subjects returned seven days later for a third blood sample and then underwent a 60-minute relaxation procedure, including visualization of the immune system, cells, and cellular activity. A fourth blood sample was obtained following this 60 -minute session. Subjects were asked to draw the mental images they experienced and were interviewed about the experience. This was followed one hour later by a fifth blood sample. Three days after the second session, a final blood sample was obtained, and each subject was interviewed to alleviate any concerns that may have occurred as a result of the study. The findings indicated no significant changes in total number of lymphocytes and NK cells, but a significant increase in NK cell function was seen following each guided imagery session compared to baseline. 
Relaxation training has also been studied in HIV-positive persons. Taylor (1995) studied 10 HIV-positive males randomly assigned to a treatment or control group. Baseline and follow-up assessment on all subjects included State Trait Anxiety Inventory, Profile of Mood States, Selfesteem Inventory, and T-cell count. Interventions for the treatment group included a combination of progressive muscle relaxation, meditation, biofeedback, and hypnotic training involving visualization. Findings reported in the study included that analysis of variance showed the treatment group had significantly more change in all measures from pre-treatment to posttreatment compared to the control group, with decreased scores on both state anxiety $(F=15.25$, $\mathrm{p}<.01)$, trait anxiety $(\mathrm{F}=17.34, \mathrm{p}<.01)$, profile of mood states and self-esteem inventory, and increased $\mathrm{T}$-cell count $(\mathrm{F}=12.65, \mathrm{p}<.01)$. The authors recognized that the sample size was small, but reported that their findings support previous findings of anxiety and distressed mood state in HIV-positive individuals.

Walker et al. (1999) conducted a clinical study specific to cancer patients undergoing chemotherapy. Ninety-six women, newly diagnosed with breast cancer, participated in the study. Half of the subjects were randomized into a control group that underwent routine chemotherapy, while the other half underwent routine chemotherapy and relaxation training involving progressive muscle relaxation and guided imagery. Both psychological and immunological assessments were performed prior to initiation of chemotherapy and repeated during the course of treatment and 6 weeks following completion. Analysis of findings indicated that women in the experimental group had less anxiety, enhanced lymphokine-activated killer (LAK) cell activity, higher numbers and percentage of activated T-cells and reduced blood levels of tumor necrosis factor alpha (TNF) 
than the women in the control group. There was no evidence of a difference in the clinical or pathological response to chemotherapy between the groups, and the clinical significance of the immune changes was not clear (Walker et al., 1999).

\section{Laughter}

Laughter or humor as a coping strategy or a means to enhance health has been a topic of recent study. A study to assess the effect of humor on the salivary secretion of IgA was conducted with 10 upper-level psychology students. Subjects provided a salivary specimen prior to and following a humorous videotape, and prior to and following viewing of a didactic videotape. Subjects rated the videotapes on a 5 -point scale ( $1=$ not at all funny to $5=$ very funny), and each subject was assessed using the Coping Humor Questionnaire. Findings revealed a significant increase in salivary concentration of IgA after viewing the humorous videotape, although the increase was short-lived. Findings also suggested that persons who used humor as a coping mechanism had higher baseline salivary concentration of IgA as compared to those who did not, leading the authors to suggest that use of humor could be incorporated as a coping strategy for health for some individuals (Dillon, et al., 1985-86).

In a study of the effects of mirthful laughter on the immune and neuroendocrine systems performed on healthy males, ten experimental subjects were exposed to a humorous videotape for one hour, while ten controls were kept in a neutral environment. All underwent blood sample collection through indwelling venous cannulation at 10-minute intervals, providing a total of 12 samples, including 3 before, six during, and 3 following the intervention time. Results indicated that, when compared to controls, subjects exposed to the humorous intervention demonstrated an 
increase in NK cell activity (Berk, Tan, Napier, \& Eby, 1989), an increase in spontaneous lymphocyte blastogenesis (Berk et al., 1988), and a decrease in the stress-associated hormones cortisol, epinephrine, dopac, and growth hormone (Berk, Tan, Fry, et al., 1989).

Lambert and Lambert (1995) studied the effect of humor on secretory immunoglobulin A (S-IgA) levels in fifth grade students. Students were randomly assigned to a group where they experienced an age-appropriate humor program or a group that participated in a non-humorous educational program. Concentrations of S-IgA were significantly increased over baseline in the humor group and remained unchanged in the control group.

In discussing the biology of humor, Fry (1994) noted that body systems that have been demonstrated by studies to be actively involved in humor are "... the muscular, the respiratory, the immune and endocrine systems, the cardiovascular, the central and autonomic nervous systems" (p. 114). He went on to note that there is increasing evidence that in most situations humor has a positive physiological effect and can be considered in general to be a positive contribution to maintenance of health.

\section{Massage and Immunity}

Back massage as a strategy for relaxation has been a topic of some nursing research. Duration of massage and variables studied vary from study to study, but nursing studies that used effleurage as the massage method reported that subjects expressed a subjective sensation of relaxation in addition to physiological or other changes (Bauer \& Dracup, 1987; Corley, Ferriter, Zeh, \& Gifford, 1995; Fakouri \& Jones, 1987; Fraser \& Kerr, 1993; Groer et al., 1994; Longworthy, 1982; Meek, 1993). Of the nursing studies specifically assessing back massage, only 
one included an assessment of immune function. Groer et al. (1994) studied the effect of 10minute slow-stroke back massage on salivary IgA levels of well, elderly adults. All study subjects completed the STAI and gave an unstimulated 5-minute sample of saliva. Following the sample collection and completion of the STAI, controls were positioned in Simms position in a bed for 10 minutes, then had a second salivary sample collected and completed the STAI a second time. Experimental subjects were positioned in the same manner and given a 10-minute slow-stroke back massage, following which they provided a second salivary sample and completed the STAI. Data were analyzed using analysis of covariance (ANCOVA). Findings of this study indicated that although anxiety levels decreased in both groups, massage did not significantly affect subjects' anxiety levels. Both control and experimental groups showed some increased concentration and secretory rate of salivary IgA, with the greater increase in the experimental group. ANCOVA showed that the variance in the experimental group, with the salivary IgA rate change at a nearly significant level, was related to the back massage. The authors concluded that increased salivary IgA concentration may result from nursing back rub and suggested that further study was indicated.

Other disciplines have conducted studies that have included back massage as one of several relaxation strategies. One study assessed the effects of relaxation on the immune system. Fifty college student volunteers were randomly assigned either to a control group, which involved touch control, or to one of four groups: guided visualization, back massage, lying quietly with eyes closed, or Benson's relaxation response. All subjects provided a saliva specimen prior to the intervention. Each intervention, including the touch-control, lasted 20 minutes, followed by a 
second saliva specimen being provided. Prior to the study, no significant difference in salivary IgA levels was found between the four treatment groups and the control group, but a significant difference was found following the intervention. Saliva assessinent showed that subjects undergoing relaxation response, visualization, or massage showed higher salivary IgA levels than did the touch control group, with massage subjects showing the highest levels of any group. The authors suggested that since salivary IgA levels may indicate local resistance to upper respiratory infection, the regular practice of relaxation strategies to increase levels may be somewhat protective against upper respiratory infections (Green \& Green, 1987).

\section{Structured Psychological Support and the Immune System}

Structured psychological support has been hypothesized as a behavioral strategy to positively affect health outcomes. Fawzy et al. (1990) studied the immediate and long-term effect on the immune system of structured psychological support, including stress management techniques, health education, problem solving skills related to their diagnosis, and group psychological support, in patients recently diagnosed with malignant melanoma. The 35-member intervention group met weekly for $1 \frac{1}{2}$ hours over the course of 6 weeks. The control group received no intervention. Baseline assessment included both psychological assessment of affective state and coping, and serum immunological assessment, including NK cell numbers and activity, LGLs, the major T-cell subsets, and major T-cell subset activation markers. These assessments were repeated at six weeks, the end of the intervention, and again at six months. Baseline comparisons showed no immunological function difference between the control and intervention groups. A significant difference between groups was found in CD57 LGLs after six weeks, with an increase 
in LGLs from baseline to six weeks in the intervention group. After six months, comparison of groups showed the intervention group demonstrating a larger increase in percent of LGLs from baseline, an increase in NK cell percentage, and a slight but non-significant increase in NK cytotoxicity. T-cell subset measurement showed a small reduction in CD4 T-cell levels. Controls showed a similar increase in NK activity. The authors suggested that a short-term group psychiatric intervention was associated with longer-term changes of the NK cell system, demonstrated primarily by an increase in the number of cells.

A somewhat similar study was conducted by Schedlowsi et al. (1994) in which 24 patients with cancer who had completed standard surgical treatment and were not undergoing chemotherapy or radiation therapy were enrolled either in an intervention group or a control group that would undergo the intervention at a later date. The experimental group participated in ten intervention sessions that occurred once a week for two hours. The intervention consisted of relaxation techniques of autogenic training and guided imagery performed at the beginning and end of each two-hour session, with education on health, stress and illness-related skill enhancement, and the "body-network" occurring between the two relaxation sessions (p. 340). Subjects were encouraged to practice the relaxation techniques at least twice a day. Blood samples were drawn before and immediately after the second and tenth sessions to assess plasma cortisol and white blood cell counts (WBC). The control group had blood samples drawn two days after the second and tenth intervention sessions participated in by the experimental group. All subjects had the same psychological and physiological assessments, which looked at coping with chronic disease, plasma cortisol levels, and WBC. Results indicated no difference in coping 
strategies as measured, but there was a significant reduction in plasma cortisol in the intervention group at the second intervention, continuing over the nine-week period. There were no significant differences in WBC absolute numbers of leukocytes and monocytes between the groups, but there was an increase in lymphocyte numbers in the intervention group after the second intervention and continuing over the nine-week period. The researchers concluded that the results supported the hypothesis that a behavioral intervention could have positive effects on immune function in breast cancer patients who had undergone surgery.

Not all studies have found a positive relationship between support and immune function. Nicholas and Webster (1996) conducted a study of the effect of a behavioral medicine program (a support group) on a variety of psychological variables and immune function in persons with HIV. Forty-eight persons were enrolled in the program, which met weekly for ten weeks. Most (79.2\%) of them were taking anti-HIV medication. CD4 counts and some other unnamed parameters were recorded pre- and post- intervention. However, the CD4 counts were measured by "self-report". There was a significant relationship found between perceived health status and CD4 counts. However, CD4 counts declined during the intervention period, especially for persons with CD4 counts less than $200 / \mathrm{mm}^{3}$, indicating that the intervention resulted in no improvement on this parameter. It should be pointed out, however, that the subjects in this study were persons with expected immunosuppression both from the disease and also as a side effect of some of the medications commonly used as treatment for HIV. 


\section{Anxiety and Chemotherapy}

An estimated half of all new cases of invasive cancer undergo chemotherapy (Otto, 1997). In the United States in 1999 there were an estimated 1,221,800 new cases of invasive cancer (Greenlee, Murray, Bolden, \& Wingo, 1999). This would result in over 600,000 persons undergoing chemotherapy that year.

Anxiety in cancer patients undergoing chemotherapy is commonly reported in the oncology-related literature (Andrykowski, 1990; Bottomley, 1997; Eller, 1999; Fredrikson et al., 1993; Jacobsen et al., 1993; Morrow et al., 1992; Rhodes, Watson, \& Johnson, 1986; Sabo \& Michael, 1996). Much of the interest in anxiety is related to findings that suggest that anxiety is related to an increased incidence of side effects such as anticipatory, as well as post-treatment, nausea and emesis (Jacobsen et al., 1993; Morrow, 1992; Rhodes et al., 1986) and anticipatory, or conditioned, immunosuppression (Bovbjerg et al., 1990; Fredrikson et al. 1993). A recent study by Newell, Swanson-Fisher, Girgis, \& Ackland (1999) found that approximately $25 \%$ of the people in their study of 201 persons with cancer had borderline or clinical levels of anxiety and depression. Depressive states were also found in about $40 \%$ of Danish patients prior to initiation of chemotherapy in a 1994 study (Middelboe, Ovesen, Mortensen, \& Bech). Jacobson et al. (1993), studying anticipatory anxiety in women receiving chemotherapy for breast cancer, found that $91 \%$ of the women stated that they were anxious prior to the first treatment. This figure dropped to $64 \%$ reporting anxiety prior to the $2 \mathrm{nd}, 60 \%$ prior to the $3 \mathrm{rd}$ and 4 th, and $57 \%$ claiming anxiety prior to the 6th treatment, indicating that at least in this study some anxiety persisted throughout treatment. 


\section{ANV as a Conditioned Response}

Bovbjerg et al. (1990) suggested that since chemotherapy induces immune suppression as well as emesis, conditioned immunosuppression may develop, in addition to conditioned nausea. In recent years, anticipatory nausea and vomiting (ANV) has been widely studied (Andrykowski, 1988).

The many side effects of chemotherapy range from uncomfortable physical symptoms, such as nausea and/or vomiting or stomatitis, to life-threatening effects, such as immunosuppression (Fischer, Knobf, \& Durivage, 1993). Distress associated with chemotherapy treatment may affect the outcome of therapy and patient compliance or continuation with the regimen (Andrykowski, 1990; Burish \& Carey, 1986; Challis \& Stam, 1992; Holland et al., 1991; Jacobsen et al., 1993), with some patients considering the side effects to be worse than the treatment itself (Pickett, 1991) and some refusing chemotherapy (Fallowfield, 1992). As a result, nausea and vomiting associated with chemotherapy has been widely studied.

Although several models for development of ANV have been proposed, it is most commonly thought to occur as a result of classic conditioning (Aapro, Kirchner, \& Terrey, 1994; Bovbjerg et al, 1992; Burish \& Carey, 1986; Challis \& Stam, 1992; Ingle, Burish, \& Wallston, 1984; Morrow, 1992; Nerenz, Levendthal, Easterling, \& Love, 1986). Chemotherapy treatment usually involves cycles of treatment that are repeated over time periods ranging from several days to several weeks. The probability of developing anticipatory nausea increases with the number of cycles received (Bovbjerg et al., 1992). ANV has been associated with emetigenicity or toxicity of the treatment, number of treatments (Aapro et al., 1994), age, and frequency of nausea and/or 
vomiting post-treatment (Cohen, Blanchard, Ruckdeschel, \& Smolen, 1986). Morrow (1992), reviewing 4 studies involving a total of 1,620 patients, concluded that the percentage of patients reporting anticipatory side effects increased with the number of chemotherapy cycles, and that after the fourth treatment, one third of patients experienced anticipatory nausea and/or vomiting. Redd and Andrykowski (1982) reported that approximately $25 \%$ of chemotherapy recipients develop ANV. A prospective longitudinal study by Challis and Stam (1992) found that of those patients who developed ANV, all had developed ANV by the time of their fifth treatment, with the average being after 3.4 treatments. Andrykowski and Redd (1987) found that $69.2 \%$ of patients who developed ANV had done so by the fifth cycle of chemotherapy, $88.5 \%$ by the sixth cycle, and $92.3 \%$ by the seventh cycle. By the eleventh cycle, all who were going to had developed ANV.

Reported incidence of ANV varies, depending on the type of chemotherapy agent involved and the method of study. Bovbjerg et al. (1992) reported a rate of ANV that ranged between $25 \%$ and $57 \%$ of patients receiving chemotherapy. Morrow (1992) reported that at least one in four patients receiving chemotherapy developed ANV. An analysis of 17 studies involving 1,700 adult and 80 pediatric patients found that $32 \%$ of the adults and $29 \%$ of the pediatric patients reported ANV (Burish \& Carey, 1986). A study of 77 women receiving chemotherapy (methotrexate, fluorouracil, vincristine, and cyclophosphamide) revealed that $57 \%$ experienced ANV (Andrykowski et al., 1988), while a study of patients receiving Cisplatin-based chemotherapy, well known for its emetic side effects, found that $65 \%$ of the patients reported anticipatory nausea in at least one situation (Coons, Leventhal, Nerenz, Love, \& Larson, 1987). A study of 149 
patients receiving chemotherapy revealed that $42 \%$ of the patients reported pre-treatment nausea, and $27 \%$ of the patients reported anticipatory vomiting (Cohen et al., 1986). In a longitudinal study, Andrykowski and Redd (1987) found that $36.6 \%$ of patients studied developed ANV. A study of ninety-five outpatients receiving mild to moderate chemotherapy regimens that excluded drugs with high emetic properties revealed that $23 \%$ developed ANV (Watson, McCarron, \& Law, 1992).

Research has indicated that the degree of patient anxiety is positively related to occurrence of chemotherapy-related nausea and vomiting, although the specific nature of the relationship remains unclear (Andrykowski \& Redd, 1992; Jacobsen et al., 1993; Jacobsen \& Redd, 1988; Morrow, 1992; Watson et al., 1992). Some studies have associated incidence of ANV with increased levels of anxiety (Andrykowski, Redd, \& Hatfield, 1985; Ingle et al., 1984; Nerenz et al., 1986; Razavi et al., 1993), whereas Coons et al. (1987) did not find an association between anxiety and increased incidence of ANV. Andrykowski et al. (1988) reported that they did not find a direct association between anxiety and initial development of ANV, although it appeared to be related to later development of ANV. Contrary to the researchers' expectations, high state anxiety was associated with delayed onset of ANV in one study conducted by Andrykowski \& Redd (1987).

Andrykowski (1990) pointed out that a limitation in the study of ANV was that state anxiety levels may increase as a result of ANV, rather than the other way around, and that there is no clear cause and effect relationship between anxiety and ANV. However, research does demonstrate a correlation between ANV and anxiety, although the relationship requires further 
study. To this end, Kvale et al. (1991) conducted a prospective study to assess autonomic reactivity, including heart rate and peripheral vascular changes, in addition to self-reported anxiety in patients undergoing chemotherapy. No difference in self-reported anxiety was found in groups with and without ANV, but a significant association was found between persons with increased autonomic reactivity and development of $\mathrm{ANV}$, leading the authors to suggest that autonomic reactivity was a more accurate marker for ANV susceptibility than self-reported anxiety. The authors also suggested that desensitization of persons with increased autonomic reactivity prior to initiation of chemotherapy may decrease incidence of ANV.

\section{Behavioral Studies to Modify Nausea and Vomiting}

ANV has shown to be resistant to drug intervention (Burish \& Jenkins, 1992). Therefore, a number of behavioral interventions have been studied as strategies for decreasing or preventing ANV. The assumption is that behavioral interventions include a cognitive component that competes with stimuli associated with chemotherapy and thus interferes with development of conditioned side effects (Vasterling et al., 1993).

Burish and Jenkins (1992) examined the effect of relaxation training (RT) involving progressive muscle relaxation and guided relaxation imagery, versus biofeedback plus RT, versus biofeedback alone, versus a no-intervention control group, on nausea and vomiting associated with chemotherapy. Antiemetic medications were used as needed. The study showed RT, in conjunction with antiemetic medication, to be effective in reducing incidence of nausea and preventing exacerbation or development of conditioned symptoms. Biofeedback was not found to have the same effect. 
Vasterling et al. (1993) studied use of relaxation training using progressive muscle relaxation or guided imagery distraction versus no intervention in chemotherapy recipients. They found that both relaxation and distraction groups reported a decreased incidence of nausea prior to chemotherapy and displayed lower blood pressure measures, as compared to controls.

A prospective study by Razavi et al. (1993) examined stress prevention versus use of alprazolam in prevention of adjustment disorders and anticipatory nausea secondary to chemotherapy. The study involved two groups using relaxation. In addition, the experimental group was given the anti-emetic alprazolam while the control group was given a placebo. Both groups were asked to practice progressive relaxation on a daily basis, and all were involved in a formal weekly or bimonthly counseling and support program. The results indicated that those using alprazolam experienced anticipatory nausea later than did the placebo control group. In both groups, the incidence of nausea was nearly the same by the completion of the fourth treatment ( $26 \%$ and $25 \%$ respectively), indicating that in this situation, the medication was not more effective than relaxation alone in preventing anticipatory nausea.

A systematic desensitization strategy, using relaxation techniques in association with systematic desensitization, was shown to be effective in reducing the incidence and severity of ANV and post-chemotherapy vomiting in a group of patients (Morrow et al., 1992; Morrow \& Morrell, 1982), while use of hypnosis and imagery intervention was reported to result in decreases in anticipatory nausea in another chemotherapy study group (Redd, Andersen, \& Minagawa, 1982). Research using guided imagery plus progressive muscle relaxation training (PMRT), 
initiated before the start of chemotherapy and during the first three treatments, resulted in prevention of conditioned nausea and was effective in decreasing severity and length of nausea following chemotherapy. In addition, relaxation training also reduced levels of autonomic arousal, as assessed by blood pressure and pulse rates, during chemotherapy treatment (Burish et al., 1987).

A number of studies have examined nausea and/or vomiting associated with chemotherapy in general, without specifying whether the symptoms were anticipatory. Lyles et al. (1982) found that relaxation training and guided imagery resulted in a significant reduction of chemotherapyrelated side effects such as anxiety, nausea, and physiological arousal in an experimental group, when compared to a group that engaged in conversation with therapists and with a control group. Another study, comparing use of an antiemetic drug regimen and relaxation, found that while the drug regimen was more effective in preventing the peak phase of vomiting, the duration of postchemotherapy vomiting was only half as long in the relaxation group, compared to the drug regimen group, suggesting that relaxation could be a useful strategy in decreasing duration of symptoms (Scott et al., 1986). Cotanch and Strom (1986) found that progressive muscle relaxation was effective in reducing anxiety, improving calorie intake, and decreasing severity of vomiting following chemotherapy.

\section{Behavioral Interventions to Modify Anxiety}

A number of behavioral interventions have been proposed as helpful in modifying anxiety. Massage and progressive muscle relaxation are two that have been reported in the literature, and will be discussed briefly, omitting literature previously discussed when addressing immune function. 


\section{Massage}

Massage has long been considered a relaxation strategy to decrease anxiety and even pain. A meta-analysis by Labyak \& Metzger (1997) of nine studies that met the criteria of backrub length of at least three minutes, pre-and post vital signs, and statistical data analysis found the following: Ten-minute massages were associated with maximum reductions in heart and respiratory rate initially, with a gradual rise to pre-treatment level during the ten-minute postmassage assessment period. Three-minute back massage was associated with the greatest reduction in both systolic and diastolic blood pressure immediately after the massage and a continued decrease for five minutes afterward. Interesting gender-related differences were reported in seven studies. Females demonstrated a rise in blood pressure during the first 3-5 minutes of the back massage, followed by a decline in blood pressure during the 10-minute rest period. Males demonstrated a decline in blood pressure after 5 minutes of massage and a continued reduction during the 10- minute rest period. Discussion suggested that massage is associated with decreased heart and respiratory rate for both male and females. The degree of decreased blood pressure seemed to be gender-related, with males showing greater overall decrease in systolic and diastolic pressure. The data indicated that "biological relaxation" demonstrated by decreased heart and respiratory rate is associated with effleurage back rubs of at least three minutes.

Field, Grizzle, Scalfidi, and Schanberg (1996) conducted a study on 32 depressed adolescent mothers who were randomly assigned to a relaxation group or a relaxation group receiving massage. The massage group received ten 30 -minute sessions of massage over a five- 
week period, while the relaxation group received ten 30 -minute periods of relaxation that included yoga and progressive muscle relaxation. Study findings indicated that the massage group showed significant decrease in state anxiety, serum and salivary cortisol levels, and pulse rate, as compared with the relaxation alone group.

Following the study of the effects of massage on depressed adolescent mothers, Field et al. (1998) studied effects of massage on 23 children with asthma. In younger (age 4-8) children, massage was associated with decreased anxiety and salivary cortisol, improved attitude toward asthma, and improved peak air flow and other pulmonary functions such as forced vital capacity, forced expiratory volume, forced expiratory flow (FEF), and peak expiratory flow. In older (age 9-14) children, massage was associated with lowered anxiety, improved attitude toward asthma, and improved forced expiratory flow.

The effects of slow stroke back massage on 30 hospice clients was also studied. Clients were given a 3-minute back massage on two days. Vital signs were measured five minutes before the massage and again immediately before the massage. The client then got the 3 -minute back massage, had vital signs measured immediately after the massage, and again five minutes later. Results from the study indicated that heart rates showed a pattern of decrease following slow stroke back massage. Systolic and diastolic blood pressure also showed a pattern of decrease. Skin temperature showed a pattem of increase. Statistically significant changes were demonstrated in all four of the dependent variables. Clinically, however, the changes were considered modest by the authors, with a difference between the highest and lowest means of 4.2 beats per minute for heart rate, $9.17 \mathrm{mmHg}$ for systolic blood pressure and $6.4 \mathrm{mmHg}$ for 
diastolic blood pressure, and 1.45 degrees Fahrenheit for skin temperature. The changes were interpreted as sufficient to indicate relaxation but not to a degree that they could put hospice patients in danger of hypotension, bradycardia, or hypothermia (Meek, 1993).

Ferrell-Torry and Glick (1993) conducted an exploratory study of the use of massage of 30-minute duration administered on two consecutive evenings to nine cancer patients experiencing cancer pain. Assessment included Visual Analogue Scale (VAS) for self-report of pain and relaxation, STAI for anxiety, and heart rate, respiratory rate, and blood pressure, done just before, just after, and 10 minutes after massage. A significant decrease in anxiety levels, heart rate, respiratory rate, and blood pressure, and increased self-report of relaxation resulted.

A prospective study with repeated measures on the effectiveness of neuromuscular release massage therapy (NRMT) on five patients with chronic obstructive pulmonary disease (COPD) was conducted over a 24-week period to study the effect on pulmonary function, respiratory muscle strength, and quality of life. Subjects underwent weekly massage with pre and post-massage pulmonary function tests obtained. The authors reported that four of the five subjects had a mean increase in thoracic gas volume and forced vital capacity, although measures did not reach statistical significance, and statistically significant improvements in forced expiratory volume, peak flow, oxygen saturation, and forced vital capacity. An improvement in heart rate was also reported, and was determined by an initial decrease of a mean of 2.96 beats per minute from the end of treatment followed by an increase of a mean of 2.85 beats per minute at the end of the entire intervention The study was limited by the small sample size, but did show statistically significant improvement of some functions after the NRMT intervention (Beeken, Parks, Cory, \& Montopoli, 1998). 
Studies have also looked at other aspects of massage. The effect of a 1-minute back rub on venous oxygen saturation in 173 critically ill patients was studied by Tyler, Winslow, Clark, and White (1990). Massage of one minute duration was chosen to simulate clinical practice. Study findings indicated that heart rate initially increased and then gradually decreased somewhat, but remained significantly higher than baseline after four minutes. $\mathrm{SvO}_{2}$ decreased by $6 \%$ immediately after massage and gradually increased to baseline by four minutes. Discussion suggested that the small changes in the heart rate and $\mathrm{SvO}_{2}$ may have been due to tactile stimulation that triggered stimulation of the autonomic nervous system, but was a minor stimulus that was well tolerated by critically ill patients. However, individual assessment of each patient's ability to tolerate position changes in order to accommodate back rub was recommended.

Massage is commonly included as a non-pharmacological measure to reduce pain and enhance relaxation (Rhiner, Dean \& Ducharme, 1996). Herr and Mobily (1992) found that simple massage and guided imagery were among five interventions receiving highest intervention content validity (ICV) scores on a 2-round Delphi study of interventions related to pain management. Massage was described as decreasing pain and producing relaxation, and simple guided imagery was described as a procedure to achieve relaxation and/or direct attention away from pain.

\section{Progressive Muscle Relaxation}

Progressive muscle relaxation (PMR) is a commonly accepted relaxation strategy. It is taught in psychiatric nursing courses (Rawlings, Williams, \& Beck, 1993; Stuart, \& Sundeen, 1995; Wilson \& Kneisle, 1992) and is suggested as a behavioral method of promoting relaxation for persons with cancer (Bindemann, Soukop, \& Kaye, 1991; Bridge, Benson, Pietroni, \& Priest, 
1988; Seligman, 1996; Simms, 1987) and other persons undergoing stressful experiences (Carey \& Burish, 1987; Mynchenberg \& Dugan, 1995). A study conducted by Arakawa (1995) with eight Japanese patients undergoing chemotherapy found an average decrease of 2.5 in stateanxiety scores of persons in a treatment group using PMR, versus a control group. The author of the study pointed out that the sample size was very small and further study is indicated. However, there seemed to be some positive effect of PMR on the Japanese chemotherapy recipients that was worth further investigation. Other studies addressing PMR as an intervention for modulating stress have already been presented in this paper when discussing neuro-immune responses to stress and modification of side effects of chemotherapy.

\section{Stress and Health}

What effects these stress-related changes have on the health of individuals is not clear. There is evidence that relates psychosocially mediated immune alterations with susceptibility to disease and disease progression, specifically with infectious illness, cancer, and progression of HIV (Kiecolt-Glaser \& Glaser, 1995). A clinical study by Cohen, Tyrrell, and Smith (1991) found that increased psychological stress was associated with increased rate of infection with the common cold. The study indicated that stress resulted in suppression of some aspects of the host resistance, possibly immune aspects, resulting in increased susceptibility to infection. The authors also noted that in some studies AIDS-infected individuals tended to differ in progression of disease based on support systems. A study by Kiecolt-Glaser, Marucha, Malarkey, Mercado, \& Glaser (1995) evaluated wound healing in persons under chronic psychological stress. Results showed a significant slowing of wound healing in the stressed individuals as compared with 
controls. After reviewing multiple studies on infectious diseases Cohen and Herbert (1996) reported that there was an association between stressful life events and increased psychological stress and increased susceptibility to respiratory infections with colds and influenza, and infection with herpes virus. However, Cohen (1995) pointed out that despite studies showing evidence for an association between psychological stress and immune responses, the relationship to health is not clear.

There have been attempts to link stress with cancer. Some reasons for trying to link psychosocial factors and cancer are outlined by Bovbjerg (1991) in the three following hypotheses:

"(1) the outcome of some cancers can be influenced by psychosocial factors,

(2) the activities of the immune system can influence the outcome of some cancers, and

(3) at least one immune response (NK cell activity), thought to play a role in defense against cancer, appears to be influenced by psychosocial factors" (p. 831).

Richardson, Landring, \& Marks (1994) reviewed 11 key studies (involving 1149 patients) of psychological factors in cancer survival. A number of methodological problems were encountered, due to varying research designs and methods, and the conclusion from the review was that there was no clear relationship between psychological factors and cancer survival. However, the authors went on to say that the area has not yet been adequately studied. The relationship between stress and the development of breast cancer was explored by Bryla (1996) through a literature review. No relationship was found between stress levels and development of cancer, although studies revealed that stress was related to having breast cancer. Stress-reduction 
strategies were helpful in dealing with stress associated with the diagnosis of cancer and improving quality of life in those affected.

Anderson et al. (1998) recently studied the role of stress and prognosis of cancer. The study involved 116 patients who had been surgically treated for invasive cancer of the breast. Study findings revealed that increased stress level was significantly predictive of decreased NK cell activity and T-cell responses, both of which are cellular immune responses that are important to cancer prognosis.

Cohen and Rabin (1998) pointed out that psychological stress can cause immune system changes of the type that can influence tumor growth and metastasis. There have been animal studies that support this concept. One study was performed on mice with Lewis lung carcinoma, in which the animals were subjected to different forms of stress, such as spatial disorientation (induced by cage spinning), physical restraint, behavioral despair (induced by tail suspension), avoidable foot shock, and maternal deprivation. The results indicated that the psychological stressors of spatial disorientation, physical restraint, and behavioral despair significantly influenced tumor metastasis in mice (Giraldi et al., 1992). These researchers also studied the effect of experimental stress on tumor response to a cytotoxic antitumor drug. Laboratory mice with Lewis lung tumor were treated with cyclophosphamide, a commonly used anti-cancer drug and a drug very effective against Lewis lung tumor. Restraint stress, which alone does not affect mouse survival, was applied as an experimental stressor. Stressed mice were found to have significant changes in $\mathrm{CD} 3+$ and $\mathrm{CD} 8+\mathrm{T}$-lymphocyte numbers. The restraint stress eliminated increased survival time from cyclophosphamide treatment, with the stressed mice showing reduced tumor 
response to chemotherapy and dying at the same rate as untreated mice. The authors proposed that the antitumor effects of cyclophosphamide may depend on a combination of drug cytotoxicity and T-lymphocyte antitumor host response, and based on their study, that stress can reduce the therapeutic effect of a cytotoxic drug (Zorzet, et al., 1998).

Another way to consider the relationships among stress, immunity and health outcomes is by looking at the Th1/Th2 balance. Cytokines fall into two main classes: the Th1 class, which stimulates the development of cellular, or cytotoxic, responses, and the Th2 class, which stimulates the development of humoral, or antibody, responses (Vedhara, Fox, \& Wang, 1999). Stress is associated with increased secretion of glucocorticoids and catecholamines. Glucocorticoids and catecholamines stimulate IL-10, a Th2 cytokine, and suppress $\mathbb{L}-12$, a Th1 cytokine, resulting in a suppression of Thl and shift toward Th2 function. "The change in Th1/Th2 balance may be involved in the stress-induced susceptibility to infectious, autoimmune, allergic, and neoplastic diseases" (Weizman \& Bessler, 1999, p. 6). Production of TH2 cytokines, especially $\mathbb{L}-10$, has been found to be elevated with a number of human neoplasias, with a corresponding reduction of Thl cytokines in many of the same neoplasms. Type 1 cytokines have anti-tumor effect, while Type 2 cytokines have an opposite effect, down-modulating tumor specific immune responses. This suggests that tumor growth could be associated with immunological changes involving cytokines reflected in an altered Th1/Th2 balance (Clerici, Shearer, \& Clerici, 1998)

Kuby (1997), discussing the effect of the balance between the Th1 and Th2 subsets on disease development and progression, gives the example of tuberculoid and lepromatous leprosy. 
Tuberculoid leprosy has a very slow progress and is characterized by a Th1-type cell-mediated response that develops to form granulomas and destruction of most of the organisms. However, in lepromatous leprosy the cell-mediated response is depressed, and a Th2-type humoral response is stimulated with resultant humoral antibody formation. Hypergammaglobulenemia may occur, and organisms are disseminated and proliferate with extensive infection and damage.

\section{$\underline{\text { Summary }}$}

Addressing anticipatory nausea and vomiting associated with chemotherapy, Burish and Carey (1986) suggested that research had been rather narrow in focusing on nausea and vomiting, and needed to broaden beyond that in studying aversive conditioning associated with chemotherapy. Using the paradigm established with study of ANV and the reported work on stress-related immunosuppression, this study addressed the potential of an intervention to modify side effects of chemotherapy, specifically to decrease anxiety, nausea and emesis, and to modify the degree of immunosuppression associated with chemotherapy and thereby enhance immune function.

The relationship between psychological and physiological stress and modulation of the immune system is well supported in research-based literature. In addition, some immune changes, such as immunosuppression, can be conditioned. Immunosuppression is associated with a number of cytotoxic chemotherapeutic agents, and Bovbjerg et al. (1990) suggested that immunosuppression may be conditioned as a response to chemotherapy.

The clinical significance of stress-related immune changes is not clearly understood, but it is hypothesized to influence physical health. In a year-long prospective study of the association 
between stress-related immune depression and health changes, Glaser et al. (1987) found an increase in the number of self-reported infectious illnesses associated with stress-related immunosuppression. The association between health and stress-related immune suppression is an area for further study.

Behavioral interventions have been shown to alter immune changes associated with stress. However, the way in which behavioral modification works on immune responses is not known (Roudebush \& Bryant, 1991) and is another area for further research.

Literature review revealed some studies addressing anticipatory neuro-immune response before a stressor and other studies addressing neuroendocrine-immune responses following a stressor. However, there are few studies addressing whether intervention can modify the neuroimmune response. Prior to 1999 , no prospective clinical studies were found that examined the effect of a relaxation intervention on neuro-immune responses of persons undergoing a psycho biological stressor. This research was intended to add to the body of nursing knowledge and to help fill a gap in the scientific literature. 


\section{Chapter III}

Methods and Procedures

\section{Overview}

The research hypotheses were studied by evaluating whether a relaxation intervention, slow-stroke back massage and progressive muscle relaxation, performed prior to initiation of and during a chemotherapy regimen, influenced neuro-immune status changes in individuals undergoing chemotherapy treatment. This chapter addresses the research design, sample identification, setting, intervention, measurements and instruments, procedure for data collection, ethical considerations, and data management.

An overview of the methodology is shown in Figure 3, and will be briefly described. Individuals who fit the sample criteria were enrolled into the study and assigned to a control group or an experimental group. Both groups underwent four doses of chemotherapy treatment, with a three-week interval between treatments. In addition to the chemotherapy, experimental subjects underwent a relaxation intervention which included a slow-stroke back massage before each dose of chemotherapy and daily practice of progressive muscle relaxation. Three neuroimmune assessments, including a panel of immune function tests, serum catecholamines, and assessment of state and trait anxiety, were conducted on all subjects. The first was a baseline assessment done prior to the first dose of chemotherapy. The second assessment was done after two doses (cycles) of chemotherapy and just before the third dose. At this point evaluation of nausea and vomiting, using the Morrow Assessment of Nausea and Emesis (MANE) was added 


\begin{tabular}{|c|c|c|c|c|}
\hline Baseline Assessment & \multirow{2}{*}{ 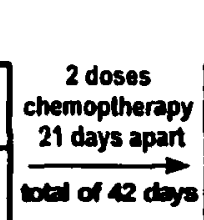 } & \multicolumn{2}{|l|}{ Second Assessment } & \multirow{2}{*}{$\begin{array}{c}\text { Third (final) Assessment } \\
\text { Just before Ath dose }\end{array}$} \\
\hline Pre-Chemotherapy Baseline & & Just before 3rd dose & & \\
\hline 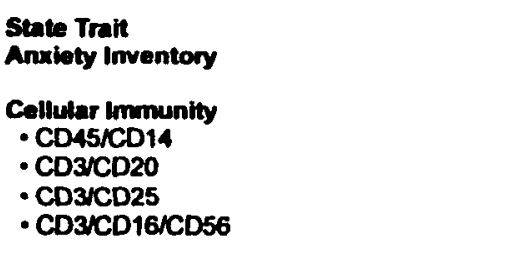 & $\begin{array}{c}\text { Control } \\
\text { Subjects }\end{array}$ & 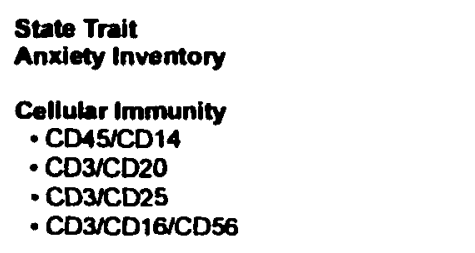 & $\begin{array}{l}21 \text { days } \\
\text { Control } \\
\text { Subjects } \\
\text { and }\end{array}$ & $\begin{array}{l}\text { State Trait } \\
\text { Anxiety Inventory } \\
\text { Cellular Immunity } \\
\cdot{ }^{C D 45 / C D 14} \\
\cdot C D 3 / C D 20 \\
\cdot C D D / C D 25 \\
\cdot C D 3 / C D 16 / C D 56\end{array}$ \\
\hline $\begin{array}{l}\text { Humoral Immunity } \\
\text { - Immunoglobulins } \\
\text { - Complement }\end{array}$ & $\begin{array}{l}\text { Experimental } \\
\text { subjects }\end{array}$ & $\begin{array}{l}\text { Humoral Immunity } \\
\text { - Immunoglobulins } \\
\text { - Complement }\end{array}$ & $\begin{array}{l}\text { Experimental } \\
\text { subjects }\end{array}$ & $\begin{array}{l}\text { Humoral Immunity } \\
\text { - Immunoglobulins } \\
\text { - Complement }\end{array}$ \\
\hline 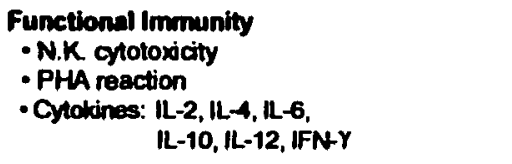 & intervention & $\begin{array}{l}\text { Functional Immunity } \\
\text { - N.K. cytotoxicity } \\
\text { - PHA reaction } \\
\text { - Cylokines: IL-2, IL-4, IL-6, } \\
\qquad \text { IL-10, IL-12, IFN-Y }\end{array}$ & intervention & 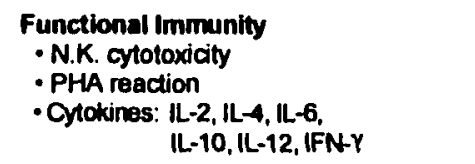 \\
\hline $\begin{array}{l}\text { Sonum Calocholaminas } \\
\text { - Epinephrine } \\
\text { - Norepinephrine }\end{array}$ & & $\begin{array}{l}\text { Serum Catecholamines } \\
\text { :Epinephrine } \\
\text { - Norepinephrine }\end{array}$ & & $\begin{array}{l}\text { Serum Catecholamines } \\
\text { - Epinephrine } \\
\text { - Norepinaphrine }\end{array}$ \\
\hline & & $\begin{array}{l}\text { plus MANE done } 30 \text { minutes } \\
\text { betore end of chemotherapy }\end{array}$ & & $\begin{array}{l}\text { plus manE done } 30 \text { minutes } \\
\text { before end of chemotherapy }\end{array}$ \\
\hline
\end{tabular}

Figure 3. Overview of methodology, showing assessment measures and intervals. 
to the assessment. The third and final assessment was done just before the fourth and final dose of chemotherapy. At this point subject participation in the study was completed.

\section{Research Design}

This study used a quasi-experimental design with an untreated control group, an experimental group, and repeated measures with pre-test and post-tests. A non-probability convenience sample was solicited from patients sequentially identified with breast cancer and scheduled to undergo an Adriamycin/Cyclophosphamide (AC) chemotherapy regimen in the outpatient department of a large university medical center. The study fit the model of a longitudinal study with a pre-test post-test control group design (Burns \& Grove, 1997).

\section{Sample Identification}

For this pilot study, a sample size of 20 was sought initially, with 10 planned for the experimental and 10 for the control group. An over sampling of $20 \%$ was expected to be included to assure an adequate sample size in the event of subject attrition (Polit \& Hungler, 1991). However, due to unforseen complications, this sample size was not feasible. The sample was made up of patients diagnosed with breast cancer and scheduled to undergo a chemotherapeutic regimen involving a combination of Adriamycin $60 \mathrm{mg} / \mathrm{m}^{2}$ and Cyclophosphamide, $600 \mathrm{mg} / \mathrm{m}^{2}$ administered intravenously (IV) every 21 days in an outpatient department. Inclusion criteria for the study were as follows. Individuals would:

1. be female

2. have a confirmed diagnosis of breast cancer

3. be at least 18 years of age 
4. be able to speak, read, and write English

5. be slated to receive intravenous AC chemotherapy on an outpatient basis Exclusion criteria for the study were as follows:

1. any previous history of cancer, or cancer at another site

2. receiving radiation treatment at time of breast cancer diagnosis

3. history of prior radiation or chemotherapy

4. smokers

5. any underlying immunosuppressive health problem

6. medical contraindication for back massage

7. already involved in systematic relaxation practice

8. receiving biological modifiers (such as granulocyte-cell stimulating factor) at time of chemotherapy institution

9. involvement in any other research protocol other than one of the medical center chemotherapy protocols

The sample consisted of females because that is the most common group identified with breast cancer. Since this was a pilot study with a small sample, including a single gender eliminated one uncontrolled variable.

\section{Setting}

The research was conducted at a large medical center with an active oncology out-patient clinic in the Faculty Medical Offices (FMO). Once identified, subjects were met by the researcher in the clinic to discuss the study, obtain a written consent and answer questions that might arise. 
Baseline physiological assessment, including blood pressure and heart rate assessment, and administration of the STAI-Y were done in the oncology outpatient clinic. Interventions for the experimental group were performed in one of the examination rooms in the oncology clinic. Environmental distractions were minimized, and the setting was controlled for temperature, noise, and privacy as much as possible. A quiet and pleasant setting away from the clinic had been set up for experimental subjects to get the massage, but when it came to the actual process, subjects preferred to stay in the clinic area for convenience and so they would not lose their parking spaces. This had advantages and disadvantages. The unique site planned for the massage would have been more pleasant and probably more relaxing in itself. However, the process of getting there and then back to the clinic and then looking for a parking space in a crowded parking lot would have been an additional stressor. In addition, as the chemotherapy occurred in the clinic setting, it was possible that the massage in the clinic setting provided a better association between relaxation and the chemotherapy treatment.

Blood samples were drawn for analysis in the outpatient clinical laboratory by qualified individuals or, if the subject had a central venous access port for chemotherapy, by a qualified registered nurse in the clinic setting. Because venipuncture is often an unpleasant experience for persons, and especially for chemotherapy patients if veins are damaged, blood samples were obtained at the time of blood collection for routine tests or at the time of intravenous cannulation for chemotherapy, in order to minimize additional venipuncture. 


\section{$\underline{\text { Relaxation Intervention }}$}

The independent variable for the experimental group was a relaxation intervention including slow-stroke back massage and progressive muscle relaxation. Slow-stroke back massage was performed according to specific criteria, based on the technique developed by Jensen-Nelson in 1948 and modified and described by Groer et al. (1994). The technique used follows (with the modification that talcum powder was used instead of warm lotion if requested by the subject, to minimize any soiling of patient clothing. If the subject preferred to keep clothing on, which was most commonly requested for comfort of the subjects, no substance was used on the hands, but the massage was otherwise performed according to the technique described).

"After warmed lotion was distributed over the back, three slow effleurage circles were made, beginning near the vertebrae and moving across the trapezius, infraspinatus, and pectoralis major muscle group. The circles continued down the lateral portion of these muscles and back toward the vertebral column. Then three circles were made in the opposite direction over the lower portion of the trapezius, infraspinatus, and upper portion of the latisimus dorsi muscles. Reversing direction again, three circles were made between the areas of thoracic vertebrae 12 and the iliac crest over the midsection of the latisimus dorsi. The massager then made three slow strokes up and down the perivertebral region of the participant's back. Lotion was added to the nurse's hands as needed, but touch was maintained throughout the procedure. The pattern was repeated for a timed 10-minute period." (Groer et al., 1994, p. 5).

Progressive muscle relaxation (PMR) guidance was provided with the use of an audio tape developed specifically for the study. For development of the audiotape, literature on PMR 
technique was reviewed (Mynchenberg \& Dugan, 1995; Rawlins et al., 1993; Sabo \& Michael, 1996; Stuart \& Sundeen, 1995; Wilson \& Kneisle, 1992), and a 30-minute PMR script was developed (see Appendix A for script). The script was reviewed by three psychiatric nursing faculty who assessed it to be appropriate. The script was read by the researcher and recorded in a professional sound studio. Instructions for the PMR included suggestions that the subject feel relaxed during chemotherapy treatments. Once recorded, soothing archived music, with no copyright constraints, was added in the studio. The completed PMR tape was listened to by three psychiatric nursing faculty and deemed to be relaxing and appropriate for PMR in terms of technique and sound. Tapes were duplicated, and all experimental subjects were provided with a PMR tape and small tape player and were encouraged to practice at home at least once daily. Subjects were asked to note the number of days and times per day they practiced PMR on an index card provided for documentation.

\section{Measurements and Instruments}

The purpose of the research was to study the effect of a relaxation intervention on anxiety, nausea and vomiting, and immune functions of persons undergoing $\mathrm{AC}$ chemotherapy for breast cancer. These drugs have identified side effects that could influence the study. Toxic side effects of both Cyclophosphamide and Adriamycin include leukopenia and nausea and vomiting (Carter, Bakowski, \& Hellmann, 1987; Fischer et al., 1993). Adriamycin is also associated with alopecia, which many patients find very stressful (Fischer et al., 1993). For the purpose of this study, hematologic and gastrointestinal side effects, specifically those involved with immunosuppression 
and nausea and vomiting, were noted. Dependent variables that were studied and recorded were anxiety, immune function, and nausea and vomiting.

Anxiety

Anxiety assessment included blood pressure and heart rate, the serum catecholamines epinephrine and norepinephrine, and state and trait anxiety.

Blood pressure and heart rate on all experimental subjects were collected before and after massage as an indicator of physiologic arousal. Blood pressure has been shown to be affected by stressful situations (Sparacino, 1982), with increase in systolic and diastolic blood pressure associated with increase in psychological stress (Halvorsen \& Vassend, 1987). Cardiovascular changes, including as a minimum heart rate and blood pressure assessment, are indicators of intensity and duration of stress, and also are related to the degree of immune change (KiecoltGlaser, et al., 1992). Because of the stress associated with diagnosis and treatment, autonomic physiologic assessment, including heart rate and blood pressure, is important when assessing chemotherapy and cancer (Nicholas \& Hollandsworth, 1986). Initially blood pressure and heart rate information was also to be obtained from the clinic records on all subjects immediately before chemotherapy infusion and during treatment. However, this information was not consistently obtained by clinic staff, so the data were incomplete.

Serum catecholamine levels were measured to assess physiologic response to a stressor. Epinephrine and norepinephrine are secreted in response to a stressor (Rabin, 1999; Robinson, 1990). Catecholamine levels may be measured in plasma, urine, and to various degrees, saliva. An increase in urine catecholamine levels has been found as a body response to stress (Kathol, Anton, 
Noyes, \& Gehris, 1989; Lundberg, 1976). However, for adequate assessment, a 24-hour urine specimen collection is needed and may prove impractical for outpatient collection. In addition, a 24-hour urine specimen is cumulative, reflecting stress over a period of time, and so would not reflect the stress of the moment when subjects were in the clinic setting. Therefore, urine collection for catecholamine levels was not considered appropriate for this study. Assessment for catecholamine levels in saliva has also been suggested as a means for physiologic assessment, but a study assessing catecholamine response to stress in saliva versus serum found that saliva levels were not indicative of a stress response, and serum catecholamine levels were of most value (Schwab, Heubel, \& Bartels, 1992). Serum assessment was used for this study as the measure with the greatest accuracy (Frankenhaeuser, 1971; Schwab et al., 1992). Specimens were collected and transported to the main hospital clinical laboratory according to laboratory protocol. There they were processed according to protocol and sent to an accredited outside laboratory for catecholamine assessment.

State and trait anxiety were assessed before chemotherapy was instituted, just before the third dose, and just before the fourth dose, using the State-Trait Anxiety Inventory, Form Y (STAI-Y) developed by Spielberger (1983) and purchased for use. This tool was designed to assess the current anxiety status of an individual, state anxiety, and how characteristically anxious an individual was, trait anxiety. The instrument has been widely used, including in studies of chemotherapy-related anxiety and stress effects on the immune system. Because anxiety states are transient, test-retest correlations for reliability are not as meaningful as the alpha coefficient for internal consistency. The STAI-Y has an across-studies reported alpha reliability coefficient of .92 
for state anxiety and .90 for trait anxiety. Concurrent validity was established through a variety of means. Use on contrasted groups revealed significantly higher scores for trait anxiety in neuropsychiatric patients as compared to normal subjects, and higher scores of state-anxiety in military recruits undergoing a stressful training program, as compared to similar age students who were not in a stressful situation. Correlation of the trait anxiety scale with other measures of trait anxiety, specifically the IPAT Anxiety Scale, the Taylor Manifest Anxiety Scale (TMAS), and the Zuckerman Affect Adjective Checklist (AACL), showed high correlations with the IPAT and the TMAS, ranging from .85 to .73 , but a moderate correlation with the AACL, suggesting that the AACL is less useful for measurement of trait anxiety. Correlations of the STAI with other, similar instruments demonstrated divergent and convergent validity (Spielberger, 1983). Okun, Stein, Bauman and Silver (1996) analyzed three commonly used psychiatric scales, the Psychiatric Symptom Index, CES-Depression Scale, and State-Trait Anxiety Scale, from the perspective of DSM-IV. Their analysis supports that all three scales are appropriate measures for anxiety and depression.

Other measures considered for this study included use of the Profile of Mood States (POMS), which assesses seven mood states, including anxiety. However, this instrument is designed to measure the current mood state as opposed to trait and current state of anxiety, as does the STAI. There is controversy whether anxious mood and state differ in significance for research. When comparing use of both instruments in the study of ANV, one researcher reported "It appears that anxiety when measured as a constellation of symptoms (such as is done on the SCL90 and the STAI) is related to the development of ANV, while anxiety measured as a mood 
(POMS) is not." (Morrow, 1992, p. S54). The Symptom Check List - 90 (SCL-90) was also considered, since anxiety is one component of the instrument; however, it is a long instrument of 90 questions, including several dimensions of which anxiety is only one, and had no obvious advantage over the STAI.

An advantage of the STAI was that both state and trait anxiety were addressed. Andrykowski (1990) had observed that a weakness in previous research was that only state anxiety had been assessed. In this study both state and trait anxiety were assessed, both prior to and following chemotherapy.

Anticipatory Nausea and Vomiting

Assessment of nausea and vomiting was performed using the Morrow Assessment of Nausea and Emesis (MANE) instrument, developed to assess for anticipatory nausea and/or vomiting (Morrow, 1984). This is currently the most widely used instrument for assessment of chemotherapy-associated nausea and vomiting addressing both anticipatory and post-treatment occurrence.

Initially the MANE author (Morrow, 1984), using 20 subjects tested 4 times, established test-retest reliability coefficients ranging from $r=.72$ to $r=.96$. Construct validity, using convergent and divergent measures, was also studied. The MANE was found to correlate more highly with measures associated with nausea and vomiting than with measures of other side effects of chemotherapy. A nonsignificant correlation was found between patients' self-report of other chemotherapy side-effects and results of the MANE, showing divergent validity. A study by Carnrike et al. (1988) of 35 outpatients receiving chemotherapy established moderate reliability 
and validity of the MANE. Test-retest reliability was established for each of the 17 items, using Pearson product moment correlations for all duration variables and Spearman rho correlations for all other variables. Test-retest reliability ranged from $r=.35(p=.04)$ to $r=.70(p=.001)$ for all non-duration variables. Duration variables ranged from $r=-.01(p=N S)$ for anticipatory emesis to $\mathrm{r}=.78(\mathrm{p}=.001)$ for anticipatory nausea.

Recently, the MANE was revised into two forms. The second form is now called the Morrow Assessment of Nausea and Emesis Follow-up (MANE-FU) and includes the original 17item instrument that assesses for nausea and vomiting in general and also includes items on ANV. This form is used for assessment of both ANV and nausea and vomiting that occurs following chemotherapy, but is designed for assessment of one treatment at a time so was not appropriate for this study, which used the original MANE instrument.

The MANE was first administered to all subjects after they had undergone two cycles of chemotherapy, and a second time at the fourth and final infusion of chemotherapy. Immune Status

Tests to assess cellular, humoral, and functional immune status were selected to assess neuro-immune response to a stressor. Blood samples were drawn, and tests were performed under a blanket order from the institution's chief oncologist. Immune function tests included Total Complement; Serum Immunoglobulin; Flow Cytometry Phenotyping of CD3 + T-Lymphocytes percent and absolute numbers, CD20+ B-Lymphocytes percent and absolute numbers, CD3+25+ IL-2 Activated T-Lymphocytes percent and absolute numbers, CD3-CD16+56+ NK Cells percent and absolute numbers, Mitogen Stimulation Assay with PHA, Natural Killer Cell Assay including 
Effectors, Target, 40:1, 20:1, 10:1, 5:1 and Lytic units, and Cytokines $\mathbb{L}-2, \mathbb{L}-4, \mathbb{L}-6, \mathbb{L}-10$, IL-12, and INFy.

Immune globulins, found in the serum, are the effector molecules of the humoral immune system and function as antibodies. Human immunoglobulins fall into five classes: IgG, IgA, IgM, IgE and IgD (Kuby, 1997; Vedhara et al., 1999). Immune globulins are an important part of the defense system against infectious agents (Rabin, 1999). IgG comprises about $80 \%$ of the total serum immunoglobulin. It coats microorganisms so that neutrophils and macrophages can recognize and destroy them (Kuby, 1997; Vedhara et al., 1999). IgG is also effective in neutralizing some bacterial toxins (Rabin, 1999). IgA, which comprises $10-15 \%$ of serum immunoglobulin, is the most common immunoglobulin in external secretions, and functions on mucous membrane surfaces to protect against pathogenic organisms (Jemmott \& McClellend, 1989; Kuby, 1997; Vedhara et al., 1999) IgM, which comprises about 5-10\% of the total serum immunoglobulin, is the first immunoglobulin class produced in response to an antigen (Kuby, 1997). Its function includes triggering of complement-mediated lysis and formation of antibodyantigen complexes (Kuby, 1997; Vedhara et al., 1999). IgE antibodies, found in small amounts in serum, are responsible for triggering release of histamines, which result in hypersensitivity reactions producing symptoms such as allergic reactions, asthma, hay fever, and anaphylactic shock (Kuby, 1997; Rabin, 1999; Vedhara et al., 1999). Little is known about the function of IgD, the final class of immunoglobulin, but it is thought to function in the activation of $B$ cells (Kuby, 1997). 
Measurement of immunoglobulins has limitations, because antigen specificity means that only certain antibodies will respond to an antigen (Vedhara et al., 1999). However, evaluation of serum immunoglobulin can provide some insight into the functioning of the humoral, or Th2, aspect of the immune system.

Complement, a major effector of the humoral branch of the immune system, consists of proteins that function to amplify the antigen-antibody response (Janeway \& Travers, 1997; Kuby, 1997). Complement activation may result in lysis, phagocytosis, and chemotaxis (Rabin, 1999). Complement measurement is also an indication of humoral, or Th2, immune system function.

Flow cytometry can be helpful in evaluating numbers and percentages of peripheral blood leukocyte subpopulations. In this research study, the percent and absolute numbers of Tlymphocytes, B-lymphocytes, activated T-lymphocytes, and NK cells were assayed. Lymphocyte subsets are commonly assessed in psychoneuroimmunology research (Vedhara et al., 1999). Findings such as those cited by Rabin (1999), who discussed multiple studies of immune alterations that occur in association with stress, and findings such as those of Dhabhar, Miller, McEwen, and Spencer, (1995) who found that laboratory induced stress resulted in changes in the numbers and percentages of leukocyte populations in peripheral blood of laboratory rats, support the enumeration of lymphocytes. Stress-induced changes in rat studies found greater decrease in numbers and percentages of B cells, NK cells and monocytes than in T cells (Dhabhar et al., 1995). Therefore, there is an advantage to cell counts based on research findings. However, limitations to cell counts include that cell numbers do not always correlate with cell function, and cell numbers fluctuate according to a number of factors, so changes may be due to physiologic effects and not stress-related (Vedhara et al., 1999). 
T- and B-lymphocytes are concentrated predominantly in the spleen and lymph nodes, with only $2 \%$ contained in blood. However, because of ease of access, blood is the most frequently used source for the study of lymphocytes in humans (Westerman \& Pabst, 1992). This study used blood samples for assessment.

Cytotoxicity is an important function of the immune system to remove foreign cells, such as tumorigenic cells or infected cells (Vedhara et al, 1999). Cytotoxicity can occur both with $T$ cells and with NK cells. NK cells are an important early response to infection, and also show nonspecific tumor cell killing functions (Kuby, 1997). A limitation in much of the research is that many studies use whole peripheral blood mononulcear cells (PBMC) as effectors, but NK cells make up only a small part of these cells. Thus cytotoxicity occurring in studies using whole PBMC as effectors may be due to effects of other cytotoxic cells as well as from NK cells (Vedhara et al, 1999). To eliminate this problem, the cells used in this study were purified NK cells, using the combined marker of CD16+ and CD56+, which is specific for NK cells. This eliminated other cell subsets that are capable of cytotoxic activity. NK cytotoxicity assay is helpful in evaluating the ability to mount a response to non-self cells and is a part of the cellular, or Thl, arm of the immune system.

Lymphocyte proliferation in response to mitogen stimulation is also a part of cellular immunity. Typically lymphocytes are stimulated with a mitogen such as PHA or CON A, resulting in cell activation and proliferation. The mitogen PHA stimulates mainly $\mathrm{T}$ cells. Proliferation responses to mitogens can inform researchers about the general responsiveness of lymphocytes, 
and if more than one mitogen is used, may show differences in general responsiveness (Vedhara et al, 1999).

Assessment of cytokine levels can be helpful in evaluating functional abnormalities in the immune system (Vedhara et al, 1999). Cytokines are soluble polypeptide proteins that signal messages to generate and augment immune responses (Weizman \& Bessler, 1999). Cytokines are key to activating B cells, cytotoxic $T$ cells, and other cells responsible for the immune response (Kuby, 1997). Although there are many cytokines now known, and more being discovered, some that fit in the Thl and Th2 groups were of major interest for this study. The Thl response involves cytokines $\mathbb{L}-2, \mathbb{L}-12$, IL-15, and IFNg, and mainly regulates cellular immunity. The Th2 response includes cytokines $\mathrm{IL}-4, \Pi \mathrm{L}-5, \mathrm{IL}-6, \mathrm{IL}-10$, and $\mathrm{IL}-13$, and mainly regulates humoral immunity (Clerici et al., 1998; Weizman \& Bessler, 1999). Activation of the Thl response results in cytokine production which activates $\mathrm{T}$ cytotoxic cells and macrophages. Activation of the Th2 response results in activation of B cells (Kuby, 1997). The Thl or Th2 responses have opposing action, so activation of one will inhibit the other (Rabin, 1999; Vedhara et al., 1999), which has significance when one looks at the Thl:Th2 balance.

This study included measurement of the Th1 cytokines $\mathbb{L}-2$, IL-12, and IFNg and the TH2 cytokines IL-4, IL-6, and IL-10. Although there are good reasons for wishing to measure cytokine levels, it is problematic in that cytokine levels are difficult to measure in healthy individuals and, according to Vedhara et al. (1999), may be most useful studied in diseased populations. Persons who are undergoing chemotherapy likely fit into that population so despite the limitations it was decided to include cytokines in the immune function measures. Immune 
function tests were performed in accredited laboratories according to standard protocol. For some tests, where there may be variability in measurements, samples were stored and all run at the same time to decrease chance of procedure related variability.

\section{Procedure and Steps of Data Collection}

Following approval by the Loma Linda University Medical Center Cancer Board, the University of San Diego Committee on the Protection of Human Subjects (see Appendix B for approval), and the Loma Linda University Medical Center Internal Review Board (see Appendix C for approval), oncology physicians and nurses in the approved outpatient oncology clinic were informed of the study, given criteria for patient inclusion and exclusion, and asked to support the study by identifying potential subjects.

\section{Enrolling Subjects Into Study}

Potential subjects were identified by an oncology physician or nurse and queried as to willingness to participate. If the person was willing to participate in the study, the researcher was paged. The researcher immediately called the site where the potential subject was and asked the nurse or physician some preliminary questions about whether the individual met criteria for inclusion in the study. If the initial criteria were met, a time was arranged for a meeting with the potential subject in the clinic, usually within a few minutes of the call. The timing of the researcher response was important because an agreement with the oncology department was that the study would not interfere with patient treatment, and chemotherapy was usually started on the same day of the clinic appointment. 
At the meeting with the researcher, subjects were given full information about the study, including the purpose of the study and what participation would involve. All subjects were given the same information at time of consent. The researcher consciously avoided including any statements that might bias a subject about the efficacy of the relaxation intervention. An informed consent (see Appendix D) was presented at the meeting, any questions answered, and subjects told that there would be no additional cost to them or change in their planned treatment regimen as a result of participation. Subjects were advised that they could remove themselves from the study at any time without penalty, and the name of an impartial contact person was provided in case of problems. Confidentiality of data was discussed, and a telephone number for the researcher was provided in the event that any further questions should arise. A signature was obtained from those agreeing to participate. Signatures were also obtained from a witness and from the researcher. Copies of the signed informed consent and the California Experimental Subjects' Bill of Rights (see Appendix E) were given to each subject in a folder that also contained copies of all data collection instruments that would be used. At the time of the explanation, subjects were informed that they would draw a slip of paper from a container for assignment either to Group A, the control group, or to Group B, the experimental group. During informed consent the researcher specifically avoided making statements that might bias the subjects to think that the experimental intervention would benefit their cancer outcome. Subjects were presented with a small box with the slips of paper as an attempt for random assignment to one of the two groups. At this point, however, the plan for randomization broke down. Some subjects said that the physician had told them that they could be "in the massage study," 
interpreting this to mean that they would get the massage. Two stated that they would happily agree to be in the study but only if they could be in the massage group, as that is what their physician told them. Others quite obviously looked into the box and choose Group B, the experimental group. Only one person followed the procedure and drew randomly from the box. For this reason the study design changed from a random to a convenience sample, so that the first seven persons who actually were able to complete the study were experimental subjects. After data collection was completed on the first seven subjects, the researcher informed potential subjects that the experimental group was filled and specifically asked the next seven persons who agreed to be in the study if they would be in Group A, the control group. Of persons who met the initial criteria, two refused when they found that they would be in the control group, and all of the five who did agree expressed a strong desire to be in the experimental group. The researcher expressed appreciation and offered that if their chemotherapy protocol included treatment (i.e. Taxol) beyond the four $\mathrm{AC}$ doses during which data collection occurred, they would be offered massage before each treatment for the remaining protocol. In addition, control subjects were told that at the completion of the data collection they would receive the PMR audio tape and tape player in the event they would like to practice PMR.

\section{Subject Assessments}

All subjects underwent the same neuro-immune assessments. The first was a baseline assessment. This was followed by two more assessments; the second after two cycles of chemotherapy and just before the third dose, and the third assessment after three cycles of chemotherapy and just before the fourth and final dose. 


\section{Baseline Assessment}

A time for baseline assessment was arranged at the initial meeting, before chemotherapy was started. Usually the assessment was on the same day as the initial meeting because chemotherapy was going to be instituted that day.

Blood pressure and heart rate were collected from the clinic record. The STAI-Y form, including both state and trait anxiety assessments, was given to the subject with the request that they complete it at that time.

A 25-ml. blood sample was obtained for stress hormone and immune function assessment. Because the contact with the subject was just before institution of the first chemotherapy treatment, the blood sample was collected when intravenous access was established for drug infusion. The blood sample was drawn in the out-patient clinic by a qualified registered nurse and did not require any additional venipuncture. Specimens were drawn in appropriate tubes (a 10-ml plain red-top and a 5-ml plain green-top tube for the immunology research lab, a 5-ml green tigertop for catecholamines and a 5-ml red tiger-top for complement and immunoglobulins), labeled, and transported by the researcher to the appropriate laboratories according to protocol.

Chart review was performed by the researcher to obtain relevant demographic data, including tumor site, stage and grade, hormone receptor status, medication history, CBC and differential, weight, and serum albumin level (see Appendix B for data collection). As needed, further information, such as any major change in dietary intake, sleep, or unexpected stress, was solicited from the subjects. Although it would have been ideal to plan neuro-immune assessment at the same time of day for each subject and at a specific time in the menstrual cycle to decrease 
hormonal or circadian effects on the immune or catecholamine tests, this was not realistic, as the timing of specimens was dependent on timing of the chemotherapy regimen. Subjects were asked to abstain from caffeinated food or drink on the day of, and not engage in heavy exercise on the day prior to collection of blood samples, as these could affect catecholamine excretion (Lundberg, 1976) and immune function (Kiecolt-Glaser, 1988; Kiecolt-Glaser et al., 1992). Subjects were not expected to fast prior to collection of blood samples.

\section{Experimental Group}

A preliminary schedule of times for massage was discussed with each subject. During the first year of the study, subjects came to the clinic the day before chemotherapy was scheduled to have blood work and for an appointment with the oncologist. At that time it was established if they would have chemotherapy the following day, based on their blood profile. The researcher came to the clinic at the time of the oncologist appointment and met with the subject and the oncology nurses, and the time was confirmed that chemotherapy would be given the next day. A telephone and pager number was provided to the subject, with the request to call the researcher in the event of any variation from the schedule. In addition the receptionist was asked to page the researcher when the patient arrived. When the experimental subjects came in for chemotherapy, they were met in the clinic by the researcher, and the massages were given. This schedule changed, however, and during the second year of data collection subjects had their blood work done, an appointment with the oncologist, and if their blood counts were high enough, chemotherapy all on the same day. This schedule was then adapted to by the researcher. 
Before the first chemotherapy cycle and just before each of the subsequent cycles of infusions, experimental subjects were given a 10-minute slow-stroke back massage. A room with an examination table, chairs, and privacy curtains was used. Initial blood pressure and heart rate were measured using equipment provided in the clinic. Subjects were offered an open-backed gown to wear, or if they chose, kept on their own clothes (usually a soft tee-type cotton shirt over a supportive brassiere). Most subjects had undergone a surgical procedure, such as biopsy, lumpectomy, or mastectomy, and found that keeping on their brassiere and clothing was most comfortable. Because of the surgical intervention, subjects were given the option of lying supine on the examination table, lying on their side, or sitting on a chair leaning over a pillow on the height adjusted examination table. Four subjects chose the supine position and three chose to sit leaning over a pillow for the massage. Slow-stroke massage was then performed for 10 full minutes by the researcher according to the specified criteria. Following the massage, subjects were asked to rate the experience on a simple scale of 1 to 4 , with $1=$ not at all relaxing, $2=$ somewhat relaxing, 3 = quite relaxing, and $4=$ very relaxing. Four categories were included on the scale to eliminate a tendency to simply choose the middle rating. The researcher showed a large card with the scale defined and asked the subject how they would rate their experience. Responses were recorded on the subject's data sheet and used in conjunction with heart rate and blood pressure values following the massage as a general indication of relaxation. The subject was covered with a bath blanket and another blood pressure and heart rate measurement obtained. The oncology nurses were then told that the subject was ready for chemotherapy and the subject was permitted to get up and leave at their discretion or when called by the nurse. 
Upon enrollment in the study, each experimental subject was given a small portable cassette machine with earphones and a tape of instructions for PMR and asked to practice PMR at least once a day. Subjects were advised that no penalty would be associated with number of times of practice, but that they were asked to report the number of times PMR was practiced. An index card with the subject's research identification number was provided to each subject, to be kept with the PMR tape for the purpose of recording the number of times PMR was practiced.

Subjects were asked to bring the card with them when they had their next appointment, and a new card was provided. If the card was forgotten, a stamped and addressed envelope was provided for mailing of the card. Duplicate cards were provided in case of loss. Subjects were asked to limit their relaxation practice to PMR for the duration of their participation in the study, or to let the researcher know of any other relaxation strategy they were using. No subjects reported use of additional relaxation strategies.

The second assessment was done just before the third chemotherapy infusion. Blood was obtained either with routine blood test or when venous access was established for chemotherapy. The STAI-Y was obtained, and in addition the subject was given the MANE questionnaire to complete. Cards recording PMR practice were collected.

The third and final assessment was the same as the second and was done just before the fourth and final chemotherapy infusion of $\mathrm{AC}$.

\section{Control Group}

The control group received chemotherapy according to the usual treatment regimen. The researcher was advised that blood pressure and heart rate was recorded before and during 
chemotherapy treatment. These data were obtained and recorded from the clinic records on all subjects when available. However, they were not consistently recorded, and because of multiple missing data were not included in data analysis

Control subjects had baseline assessment performed in the same manner as experimental subjects. For the second and third assessment, the STAI-Y and MANE were given to the subject prior to their clinic appointment, and the subject was requested to fill out the STAI-Y and the MANE in the clinic on the day of chemotherapy and place it in a provided addressed envelope. If subjects forgot the questionnaires the researcher met very briefly with them to provide copies and provide an addressed envelope. Blood samples were obtained either with the routine blood work or when intravenous access was established for chemotherapy. The researcher made arrangements ahead of time for the blood draw and also was available to collect and transport specimens immediately.

\section{Completion of Participation}

After the final assessment, all subjects were advised that their participation in the study was completed. They were asked if they had any questions about the study and, if so, these were addressed. They were thanked for their participation and again offered a summary of the findings once the study was completed

\section{Ethical Considerations}

An ethical consideration was the cost of study versus potential benefit. Even at decreased costs for a research study, the cost of blood analysis alone for catecholamines and immune studies was significant. The cost was covered by the researcher, so no additional cost was experienced by 
subjects in the study. The potential benefit was that if interventions could be found that decrease the side effects of anxiety, nausea and vomiting, and immunosuppression associated with chemotherapy, this may have a significant impact on the health of individuals undergoing chemotherapy. An incidental potential benefit was that the effect of AC combination chemotherapy on immune function has been reported for a number of immune functions but not for the combination of tests used in this study, so there will be information available on the effect of AC chemotherapy that may be helpful for future treatment planning.

Maintaining the confidentiality of participants while also relaying results of the immune studies to their physicians was not a significant problem, as data were handled according to existing methods of laboratory reporting. Confidentiality of persons in the study was maintained by recording data on number-identified forms. The cross-reference and any identifiable information was kept in a locked file in the office of the researcher. Neuro-immune studies were collected according to patient name and hospital identification number and were reported to primary physicians in that manner. If a subject had asked about the results, she would have been referred to her primary physician. The same information was recorded without name on a number identified form.

There were no anticipated risks associated with this study. Potential risk to participants due to data collection via venipuncture was not significant because specimens were obtained with routine venipuncture. The psychological risk was unknown. It was possible that persons newly diagnosed with cancer could be further stressed by participation in the research study. It is also possible that some could find participation a positive experience. All experimental subjects 
volunteered that the PMR tape was very relaxing and that the massage was relaxing. Control subjects expressed no negative feedback. The fact that all persons asked to participate in the study expressed strong desire to be in the experimental group, despite being advised that the effect of the intervention was unknown, posed a potentially ethical concern about "denying" a person participation in something that they thought might be beneficial. In further study of this nature it may be important to consider having control subjects in a delayed entry status so they would anticipate having the intervention at a later time when it would not affect data collection.

\section{Data Management}

A system of coding data, using pre-categorization for the demographic data on the questionnaire was established prior to data collection. Upon completion of data collection, data were cleaned and coded. Missing data were handled by assignment of an unused number (Polit \& Hungler, 1991).

A statistician was consulted prior to data collection and for data interpretation. Data were analyzed statistically to assess for differences within groups and differences between groups. Within-group differences to be studied included differences between immune function tests, anxiety measures, and serum catecholamine levels from beginning, second, and final analysis. Analysis also included correlation studies of the dependent variables immunosuppression, ANV, and anxiety. Subjects acted as their own controls.

Not all sample sets were complete. Heart rate and blood pressure obtained before and during chemotherapy were initially planned for inclusion, but these were not obtained consistently in the clinic, and because of the amount of missing data could not be included in analysis. 
Beginning and ending blood samples were drawn on all but one experimental subject, who was transferred to another facility before the final assessment. However the middle assessment was missed on a number of subjects due to problems with obtaining blood either in the laboratory or in the clinic, or in three cases, because of subject refusal; one refused because she had her blood drawn in the emergency room and didn't want any more drawn, one because she was considering dropping out of the study although she decided to stay in, and the third because she was not feeling well and said that she was afraid that any additional blood draw would make her feel weaker. In addition, some values were missing because of laboratory error in processing the specimens.

There was also considerable variation among experimental subjects in practice of PMR. Subject use of PMR was categorized into three groups. Final evaluation revealed that two of the experimental subjects did not practice PMR at all after the first two or three times and were categorized in a no practice (no PMR) group. Two subjects practiced PMR $10-50 \%$ of the time and were categorized as moderate use (moderate PMR), and three of the subjects practiced PMR $50 \%$ of the time or more and were categorized as high use (high PMR). 


\section{Chapter IV}

\section{Findings}

The following research hypotheses and goals were addressed. A brief summary of the findings is reported with each.

Hypothesis 1: Relaxation intervention, in the form of slow-stroke back massage and progressive muscle relaxation, would reduce the degree of anxiety in clients receiving chemotherapy, as compared to those who did not experience the relaxation intervention. This hypothesis was partially supported by one measurement: State anxiety was significantly lower at measure 2 for the experimental group $(t=3.20, p=0.02)$.

Hypothesis 2: Relaxation intervention, in the form of slow-stroke back massage and progressive muscle relaxation, would reduce the degree of anticipatory nausea and vomiting (ANV) in clients receiving chemotherapy, as compared to those who did not experience the relaxation intervention. This hypothesis was not supported, and no significant difference in anticipatory nausea and vomiting was found between groups.

Hypothesis 3: Relaxation intervention, in the form of slow-stroke back massage and progressive muscle relaxation, would reduce the degree of immunosuppression in clients receiving chemotherapy, as compared to those who did not experience the relaxation intervention. This hypothesis was not supported by the limited amount of usable data generated by the research.

Because of the lack of information on bio-behavioral intervention and chemotherapy, additional goals of this pilot study were to expand on the available information of the effect of Adriamycin/Cytoxan (AC) chemotherapy on the immune system and to develop a plan for a 
further intervention study of persons undergoing chemotherapy. The goals of this pilot study were met. Clinical and immunological information was gained on what to expect with A/C chemotherapy, providing a basis on which to plan a further study.

\section{Sample Description}

A quasi-experimental design was used with a convenience sample of women diagnosed with breast cancer and scheduled to undergo chemotherapy, assigned either to a control or an experimental group. The final sample included twelve subjects: five in the control group and seven in the experimental group. Six of the experimental group subjects completed the study, and a seventh subject completed all but the final evaluation and then was lost because of transfer to another facility for stem cell transplant therapy.

All subjects were female with a confirmed diagnosis of breast cancer and were scheduled to receive four cycles of chemotherapy with Adriamycin $60 \mathrm{mg} / \mathrm{m}^{2}$ and Cyclophosphamide 600 $\mathrm{mg} / \mathrm{m}^{2}(\mathrm{AC})$ administered intravenously every 21 days. The subjects were between the ages of 33 and 58 , with a mean age of 46.9 .

Baseline neuroimmune assessment was performed prior to the first dose of chemotherapy. A second assessment was performed after two cycles and just before the third cycle. A third and final assessment was done after three cycles and just before the fourth (final) cycle. Subjects in the control group received AC chemotherapy according to usual regimen. Subjects in the experimental group also received chemotherapy according to usual regimen, and, in addition, received a 10-minute slow stroke back massage just before each chemotherapy treatment and were asked to practice guided PMR on a daily basis. 


\section{Presentation of Findings}

Data were analyzed using descriptive statistics, correlational statistics with stepwise multiple regression and pair-wise deletion, and Analysis of Covariance (ANCOVA). An alpha level of 0.05 was used to indicate statistical significance.

For each group, t-tests were performed on variables to assess for significant difference between the control and experimental groups. In addition, ANCOVA was performed on all of the assessment measurements to explore whether the experimental group and control group differed to a greater degree than one would expect by chance. ANCOVA was also used because of the lack of random assignment of subjects (Munro \& Page, 1993). A Pearson correlation matrix using step-wise regression with paired deletions was also performed, but this was really a theoretical exercise since the number of subjects was below what would be needed for significance.

The sample size in this pilot study was small, requiring cautious interpretation of study findings. However, when considering the clinical nature of the study and the tests involved, important information was gained that will help in planning for future study.

Findings are presented according to each hypothesis category of anxiety, ANV, and immune function. These are followed by a discussion of the findings, and then the goals of the study are addressed with a plan for further study.

$\underline{\text { Anxiety }}$

Measurements for anxiety included state and trait anxiety inventories (STAI) and the catecholamines epinephrine and norepinephrine. Blood pressure and heart rate were not analyzed 
statistically because of missing data. Basic descriptive statistics with t-tests of mean differences are shown in Table 1.

There was one statistically significant finding with the anxiety data. The state anxiety mean was significantly lower in the experimental group at Measure $\# 2$ when compared to the control group $(\mathrm{t}=-3.20, \mathrm{p}=0.02)$. At baseline and end measures the groups had no statistically significant difference.

Table 1

Descriptive Analysis of Anxiety Measures

\begin{tabular}{|c|c|c|c|c|c|c|}
\hline $\begin{array}{l}\text { Test and Sample \# } \\
\text { (Reference Range) }\end{array}$ & $\begin{array}{l}\text { Mean } \\
\text { of All } \\
\end{array}$ & $\begin{array}{r}\text { SD } \\
\text { All } \\
\end{array}$ & $\begin{array}{l}\text { Mean for } \\
\text { Control }\end{array}$ & $\begin{array}{l}\text { Mean for } \\
\text { Experimental }\end{array}$ & \multicolumn{2}{|c|}{ t-score $p$} \\
\hline \multicolumn{7}{|l|}{ Epinephrine (<60 pg/mL) } \\
\hline Measure \#1 (baseline) & 14.200 & 15.598 & $12.750(n=4)$ & $15.167(n=6)$ & 0.227 & 0.826 \\
\hline Measure \#2 & 17.667 & 17.614 & $28.333(\mathrm{r}$ & $7.000(n$ & 1.773 & 0.151 \\
\hline Measure \#3 & 12.556 & 9.735 & $12.750(n=4)$ & $12.400(n=5)$ & 0.050 & 0.961 \\
\hline \multicolumn{7}{|c|}{ Norepinephrine $(120-680 \mathrm{pg} / \mathrm{mL})$} \\
\hline Measure \#1 (baseline) & 536.900 & 329.494 & $687.250(n=4)$ & $436.667(n=6)$ & 1.207 & 0.260 \\
\hline Measure $\# 2$ & 523.833 & 261.796 & $671.667(n=3)$ & $376.000(n=3)$ & 1.574 & 0.190 \\
\hline Measure \#3 & 442.333 & 198.144 & $561.250(n=4)$ & $347.200(n=5)$ & 1.832 & 0.110 \\
\hline \multicolumn{7}{|l|}{ State Anxiety Score (20-80) } \\
\hline Measure \#1 (baseline) & 45.833 & 13.960 & 43.2( & 47.7 & 0.534 & 0.600 \\
\hline Measure \#2 & 42.850 & 12.389 & $51.000(n=4)$ & $32.000(n=3)$ & 3.200 & $0.02 *$ \\
\hline Measure \#3 & 37.182 & 11.898 & $37.000(n=5)$ & $37.333(\mathrm{n}=6)$ & 0.043 & 0.960 \\
\hline \multicolumn{7}{|l|}{ Trait Anxiety Score (20-80) } \\
\hline Measure $\# 1$ (baseline) & 39.083 & 13.104 & $36.400(n=5)$ & $41.000(n=7)$ & 0.581 & 0.570 \\
\hline Measure \#2 & 35.286 & 9.232 & $39.250(n=4)$ & $30.000(n=3)$ & 1.417 & 0.210 \\
\hline Measure \#3 & 38.545 & 8.937 & $38.000(n=5)$ & $39.000(n=6)$ & 0.175 & 0.860 \\
\hline
\end{tabular}

*significance at $<.05$

Although the state means were slightly higher for the experimental group at baseline and higher for the control group at Measure $\# 2$, Measure \#3 means were similar in both groups. 
Trait means were higher at baseline in the experimental group and higher at measure \#2 for the control group, but these are not supported statistically.

Table 2

Analysis of Covariance for Anxiety Measures

\begin{tabular}{|c|c|c|c|c|}
\hline \multirow{2}{*}{$\begin{array}{l}\text { Test and assessment } \\
\text { Time }\end{array}$} & \multirow{2}{*}{$\begin{array}{l}\text { Mean for } \\
\text { Controls }\end{array}$} & \multirow{2}{*}{$\begin{array}{l}\text { Mean for } \\
\text { Exp. Grp }\end{array}$} & \multicolumn{2}{|c|}{$\begin{array}{c}\text { Tests of Between-Subjects } \\
\text { Effects }\end{array}$} \\
\hline & & & $\mathrm{F}$ & Sig \\
\hline \multicolumn{5}{|l|}{ Epinephrine $(<60 \mathrm{pg} / \mathrm{mL})$} \\
\hline Measure \#1 baseline) & \multicolumn{2}{|c|}{ Not calculated (d/ pairwise deletion) } & & \\
\hline Measure \#2 & $13.397(n=3)$ & $13.953(n=3)$ & \multicolumn{2}{|c|}{ not calculated } \\
\hline Measure \#3 & $13.667(n=3)$ & $13.750(n=4)$ & 0.003 & 0.959 \\
\hline \multicolumn{5}{|l|}{ Norepinephrine $(120-680 \mathrm{pg} / \mathrm{mL})$} \\
\hline Measure \#l (baseline) & $555.500(n=3)$ & $230.000(n=4)$ & & \\
\hline Measure $\# 2$ & $800.500(n=2)$ & $374.000(n=3)$ & 0.123 & 0.759 \\
\hline Measure $\# 3$ & $549.000(n=3)$ & $282.000(n=4)$ & 5.453 & 0.080 \\
\hline \multicolumn{5}{|l|}{ State Anxiety Score (20-80) } \\
\hline Measure \#1 (baseline) & $44.250(n=5)$ & $34.500(n=6)$ & & \\
\hline Measure \#2 & $51.000(n=3)$ & $33.000(n=4)$ & 11.594 & $0.027^{*}$ \\
\hline Measure \#3 & $33.500(n=5)$ & $32.000(n=6)$ & 0.008 & 0.930 \\
\hline \multicolumn{5}{|l|}{ Trait Anxiety Score (20-80) } \\
\hline Measure \#1 (baseline) & $39.250(n=5)$ & $32.000(n=6)$ & & \\
\hline Measure \#2 & $39.250(n=3)$ & $31.500(n=4)$ & 0.077 & 0.795 \\
\hline Measure \#3 & $37.500(n=5)$ & $30.000(n=6)$ & 0.031 & 0.865 \\
\hline
\end{tabular}

*significance at $<.05$

With ANCOVA (see Table 2), state anxiety scores were also found to be significantly lower at the middle assessment, Measure $\# 2$, in the experimental group $(F=11.59, p=.027)$ as compared to the control group. No difference was found between the groups at the final measure.

Examination of the norepinephrine means, shown in Table 1, suggests that the control group had higher norepinephrine means at all assessments, and particularly at the middle 
assessment. However because of the small sample size and variation within groups, no statistical significance was found. When groups were adjusted with ANCOVA for baseline differences the differences flatten out and show no significant difference between groups at midpoint $(F=.123$, $p=0.759)$, but they do show a possible trend towards a significant difference at end measure, with the experimental group having a lower mean than the control group $(F=5.453, p=0.08)$, (see Table 2). These data have to be regarded with caution because of the limited sample size. Although the numbers were small, a Pearson Product Moment Correlation Coefficient was run to find if there was any correlation between norepinephrine level and anxiety score. The Pearson correlation matrix showed insignificant association between norepinephrine level and state anxiety $(r=-0.27)$ and Trait anxiety $(r=-0.22)$. No significant differences or trends were found with the trait anxiety or epinephrine measurements on either t-test or ANCOVA.

Although the sample size was too small to expect any statistically significant findings, the researcher was interested to see if there were any changes that, with a larger sample, might indicate differences associated with amount of PMR use. Anxiety measures were depicted graphically according to whether the subject was in the control or experimental group, and according to amount of PMR practice. These data will be discussed only in terms of the pattern seen on the graphic depiction and are not considered statistically significant. However, they may be useful when planning further study. When PMR practice and state anxiety scores were evaluated, as graphically depicted in Figure 4, a pattern was seen indicating a greater degree of drop in state anxiety scores in persons who had moderate to high PMR use as compared to controls. Interestingly the experimental group who did not practice PMR showed the lowest end 


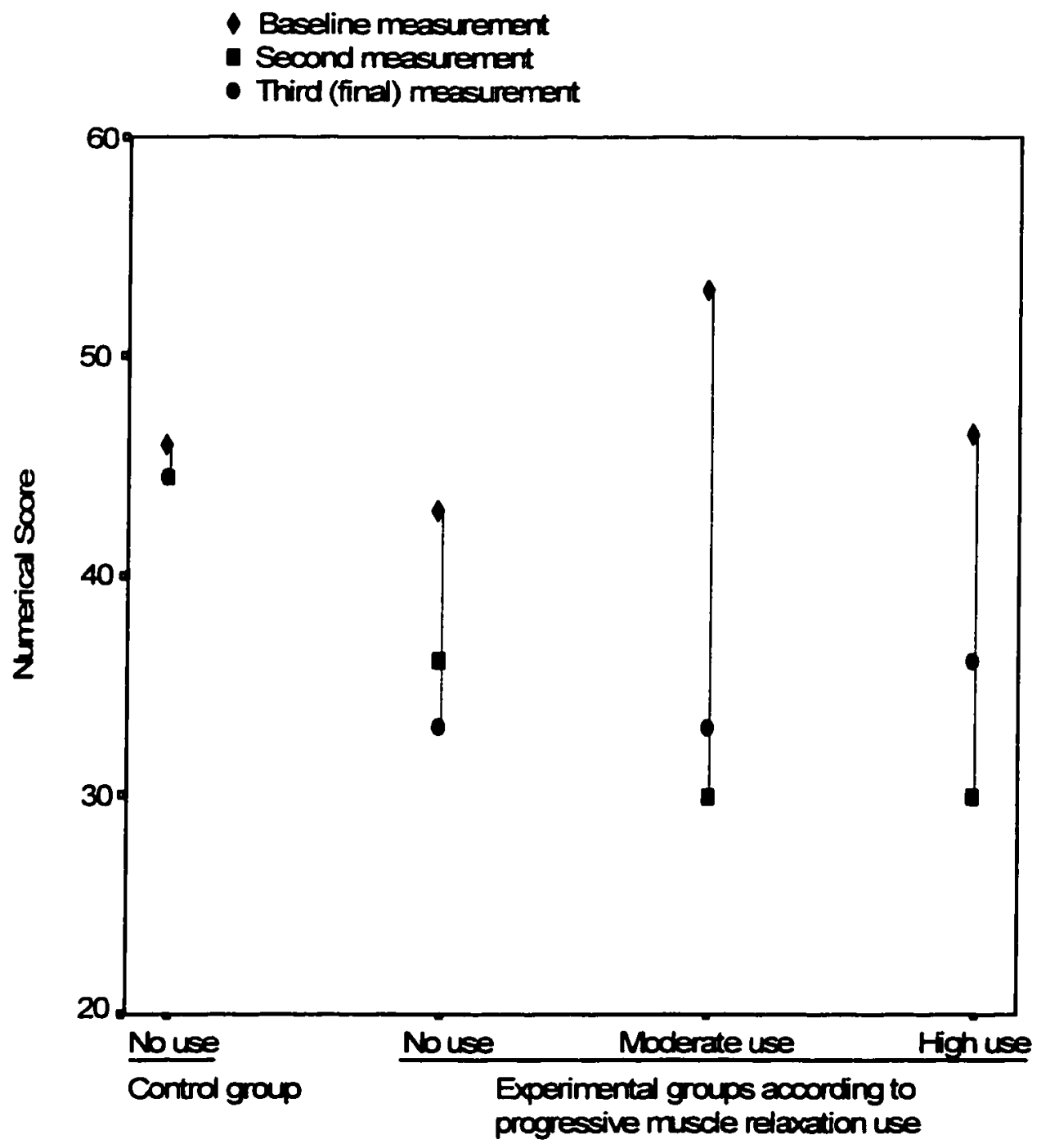

Figure 4. State anxiety scores at three different intervals according to group and amount of PMR practice. 
measurement score. This may be because of personality characteristics that may have contributed to their not following the PMR regimen, and it would be of interest for further study. When looking at trait anxiety score means and PMR use (see Figure 5), trait score means showed an increase from baseline in the control group and also in the experimental group with high PMR use, although the experimental group had lower baseline and ending trait score mean with a drop at Measure \#2 compared to the control group. In the experimental groups with no PMR use and moderate PMR use, the trait score dropped from baseline to end measurement, with the moderate PMR use group showing a further drop at Measure \#2.

Overall, the experimental groups had lower end point trait and state score means than did the control group. The experimental group that did not practice PMR started out with the lowest trait anxiety score mean. It would be interesting to study whether this is associated with personality factors that reflect a more relaxed attitude in general on stress measurement instruments.

Analysis of norepinephrine level means and groups according to use of PMR, shown in Figure 6, showed an interesting pattern. Control subjects and experimental subjects who did not use PMR showed an increase from baseline in Norepinephrine level means, while experimental subjects who used moderate to high practice of PMR showed a decrease from baseline in Norepinephrine level means. The high use PMR group had a drop from baseline to measure two, and then went up for the third measure. The high use PMR group started out with the highest baseline and remained higher than the other groups through all measures. A larger sample size might reveal different results and would be of interest to study further, and to evaluate whether 


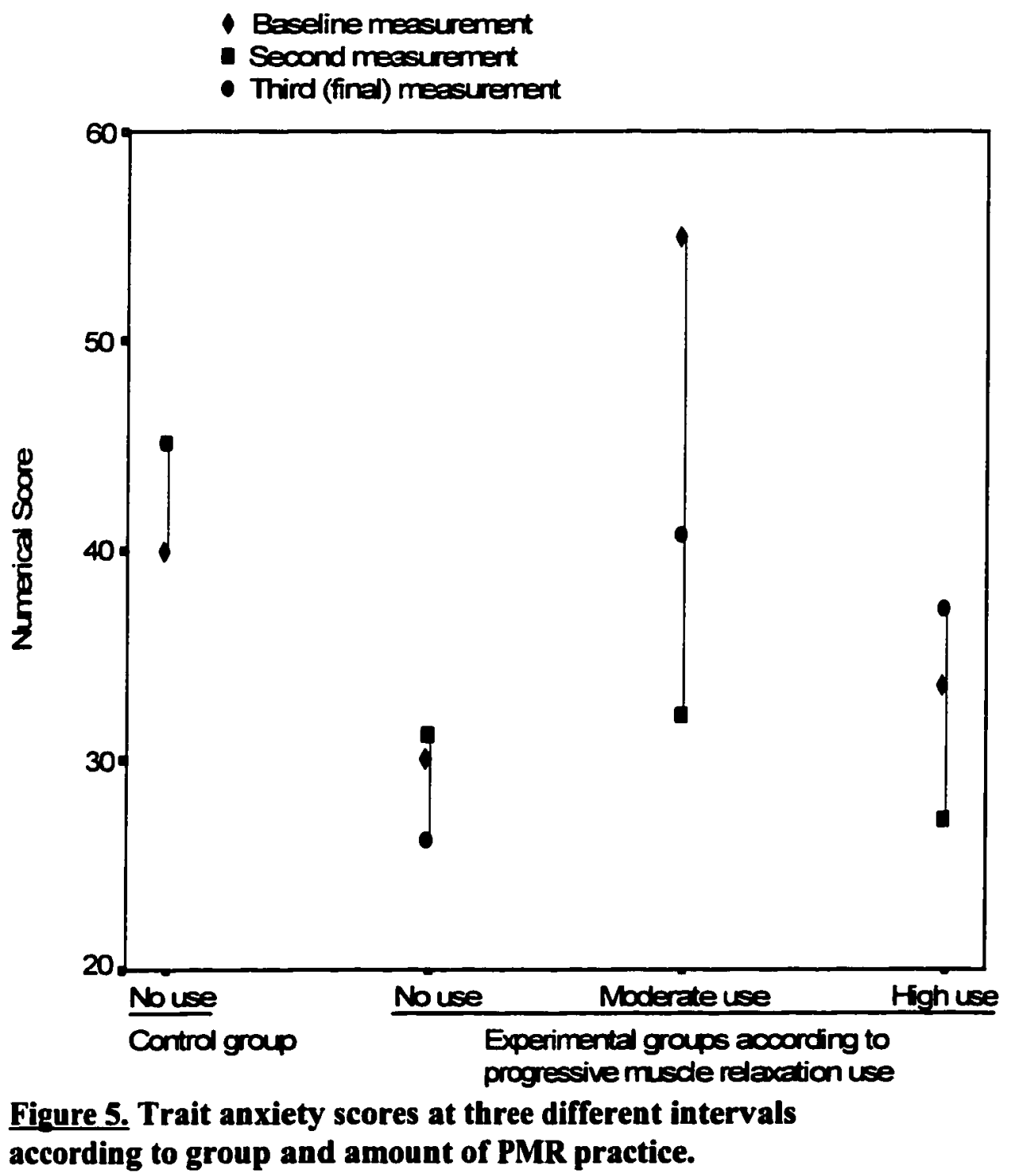




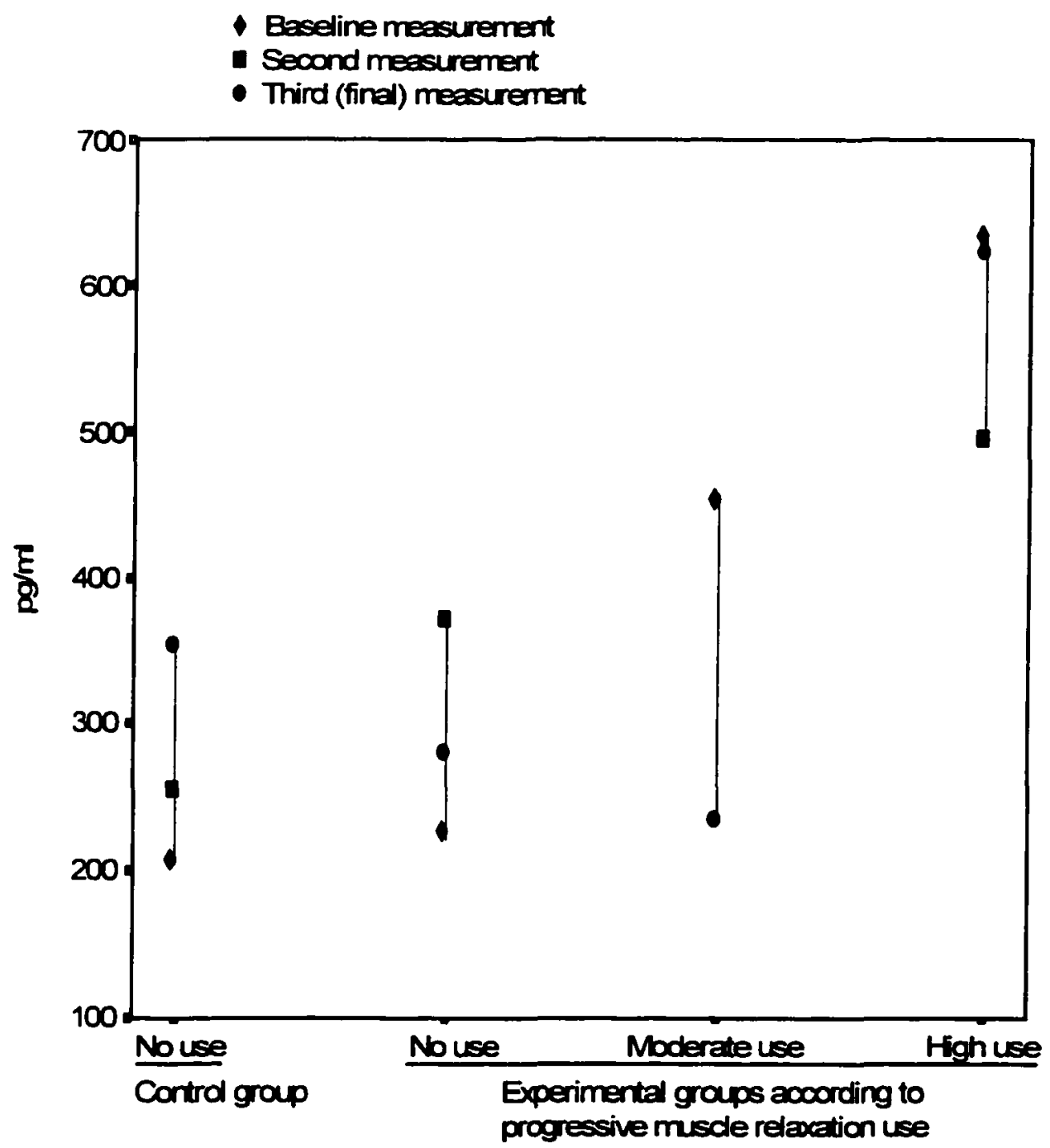

Figure 6. Norepinephrine levels at three different intervals according to group and amount of PMR practice. 
there are personality factors that are associated both with anxiety level and high use of a strategy such as PMR. While the findings must be regarded cautiously, they do raise the possibility that there may be differences found based on amount of PMR use and anxiety measures and would be of interest for further study.

\section{Anticipatory Nausea and Vomiting}

Occurrence of nausea and vomiting was assessed using the MANE instrument. The MANE was administered at the second and third measurement during the chemotherapy regimen, because the instrument was designed to evaluate chemotherapy associated nausea and emesis. Each MANE instrument assessed for nausea and/or emesis occurring after the previous chemotherapy cycle and also prior to the cycle the subject was undergoing.

Table 3

Number of Subjects Who Reported Nausea and/or Emesis on MANE

\begin{tabular}{|l|l|l|l|}
\hline \multicolumn{1}{|c|}{ Group } & Before cycle 3 & After cycle 3 & Before cycle 4 \\
\hline Experimental Group $(\mathrm{n}=6)$ & 1 out of 6 & 5 out of 6 & 0 out of 6 \\
\hline Control Group $(\mathrm{n}=5)$ & 2 out of 5 & 4 out of 5 & 1 out of 5 \\
\hline $\begin{array}{l}\text { Difference between groups } \\
\text { using Chi Square Analysis }\end{array}$ & $\begin{array}{l}\mathrm{X}^{2}=0.75, \mathrm{df}=1, \\
\mathrm{p}=0.39\end{array}$ & $\begin{array}{l}\mathrm{X}^{2}=0.02, \mathrm{df}=1, \\
\mathrm{p}=0.89\end{array}$ & $\begin{array}{l}\mathrm{X}^{2}=1.32, \mathrm{df}=1, \\
\mathrm{p}=0.25\end{array}$ \\
\hline
\end{tabular}

Analysis of the MANE results obtained showed no significant difference between groups in terms of anticipatory or post-chemotherapy nausea and vomiting (see Table 4). This is not to say that nausea and/or emesis did not occur in the subjects. The AC regimen is known to be emetogenic, and rigorous management with pre- and post-chemotherapy medication was included 
in the patient treatment regimen. Nausea and/or emesis occurred most commonly after chemotherapy in all of the study subjects, although there were some reports of ANV primarily in the control group. The number in each group was small, and even though there might be a difference anticipated when looking at the raw data, it was not sufficient for statistical significance.

Immune Function

Immune function was assessed with tests in six major categories, some of which had multiple measures. Immune function tests included Total Complement, Serum Immunoglobulins, Flow Cytometry Phenotyping that included CD45-F/CD12-PE lymphocyte purity, CD3+ Tlymphocytes (percent and absolute numbers), CD20+ B-lymphocytes (percent and absolute numbers), CD3+25+ $\mathbb{L}-2$ Activated T lymphocytes (percent and absolute numbers), CD3CD16+56+ NK Cells (percent and absolute numbers), Mitogen Stimulation Assay with PHA at three concentrations, Natural Killer Cell Assay including Effector cells, Target cells, cell death at 40:1, 20:1, 10:1, 5:1 and Lytic units, and the Cytokines $\mathbb{I L}-2$, $\mathbb{L}-4, \mathbb{L}-6$, $\mathbb{L}-10, \mathbb{L}-12$, and $\mathbb{N} F y$. Initially, the results of all of these immune function test results were analyzed. However further evaluation revealed that three of five control subjects (60\%) and two out of seven experiment subjects (28\%) required Neupogen, a granulocyte cell-stimulating factor(G-CSF), for neutropenia. Chi-Square analysis was done to evaluate if there was any significant difference in the requirement for Neupogen use between groups. No significant difference was found $\left(X^{2}=1.185, \mathrm{df}=1, \mathrm{p}=.276\right)$. However, because of the immune system stimulation effect that G-CSF has, results obtained on subjects who required Neupogen were unusable in this study. The results on subjects who did not require G-CSF 
were analyzed using descriptive statistics simply to see if any trends might emerge despite the very small sample size (see Appendix $G$ for descriptive statistics). There were few statistically significant differences in immune tests found between the control and experimental groups, and those that were found must be viewed with great caution because of the very small sample size, particularly for the control group at measure \#2 which included a sample size of one.

Evaluation of immunoglobulins showed that at the final measure, measure \#3, the mean for IgM was higher for the experimental group $(179.50 \mathrm{mg} / \mathrm{dl})$ compared with the control group (89.45 $\mathrm{mg} / \mathrm{dl})$ and approached significance $(\mathrm{t}=-2.557, \mathrm{p}=.063)$. No other differences were seen with the immunoglobulins. Total Complement could not be evaluated because of missing data.

Evaluation of Lymphocyte sub-sets showed a possible trend of T Lymphocyte CD3+ percent being higher in the control group at Baseline $(t=2.315, \mathrm{p}=0.69)$, but this pattern was not reflected in the T Lymphocyte CD3+ absolute numbers, where no significant difference was found between groups. The IL-2 Activated Lymphocyte percent was significantly higher in the single control subject at Measure $\# 2$, with a mean of $53 \%$ as compared to $16.6 \%$ for the three experimental subjects $\left(t=12.503, p=.006^{*}\right)$. IL-2 Activated Lymphocyte absolute numbers looked slightly higher in the single control subject at Measure \#2 as compared with the experimental subjects, with means of 0.580 and 0.203 , respectively, but the difference was not statistically significant $(t=3.225, p=.084)$. No significant difference was seen between the groups with B Lymphocyte CD20 cells or NK Cells.

No statistically significant difference was found in the NK Assays of percent cell death. The means for the control group and experimental group at baseline measure were $7.7 \%$ and 
$29.6 \%$, respectively $(t=-1.335, p=.089)$, and on other assays some means seemed to be higher in the experimental group than in the control group, but no statistically significant differences were found. At Measure \#2 a significant difference was seen in Target Cells, with the single individual in the control group having a significantly higher level than the three individuals in the experimental group $\left(\mathrm{t}=12.950, \mathrm{p}=.006^{*}\right)$. Because this involved a sample of one in the control group, the meaning is questionable.

The final category of tests evaluated involved selected cytokines. The cytokine IFN gamma was evaluated, but the results were reported as zero in all of the subjects, so no statistical evaluation was performed for this test. The cytokine IL-10 was also evaluated, but the test was repeated when results appeared suspicious, and when they again appeared suspicious, the kit manufacturer was contacted. It was discovered that the kit was contaminated, so the information was unusable, and there was no remaining sample for repeating the test. IL-2 was assessed on all subjects. Only one sample out of the 30 IL-2 samples processed resulted in any level of IL-2 being detected. This one positive sample gave a reading of $11.4 \mathrm{pg} / \mathrm{ml}$, very much out of the expected range (the usual reference range for IL-2 is $0-1.6 \mathrm{pg} / \mathrm{ml}$ ) and it was concluded that this finding was not valid. The researcher thus concluded that there were no IL-2 levels detected in the control or experimental subjects.

There were no significant differences found between groups with IL-4 and IL-12. However, at Measure \#2 the single individual in the control group had a higher IL-6 mean (4.32 $\mathrm{pg} / \mathrm{ml})$ than the experimental group mean $(1.77 \mathrm{pg} / \mathrm{ml})$, and this was statistically significant $(\mathrm{t}=$ $11.602, \mathrm{p}=.007)$. No conclusion can be drawn from this finding because of the limited sample size. 


\section{Discussion of Findings}

Although statistically significant findings were limited, there are some interesting patterns to be seen in this pilot study that would suggest further study. The findings give direction and raise questions that are important for future research in this area.

\section{Anxiety}

Anxiety is commonly associated with chemotherapy. In 1999 , over 600,000 persons were estimated to have undergone chemotherapy in the U.S.A., so there is the potential for a large number of individuals to be affected. Ninety-one percent of the women studied by Jacobson, Bovbjerg, and Redd (1993), stated that they were anxious prior to their first chemotherapy treatment. This figure dropped to $64 \%$ reporting anxiety prior to the $2 \mathrm{nd}, 60 \%$ prior to the $3 \mathrm{rd}$ and 4 th, and $57 \%$ reporting anxiety prior to the 6 th treatment. Other studies have reported anxiety and depressive states ranging from $25 \%$ to $40 \%$. Based on data of this nature, this study was undertaken with the understanding that the process of undergoing chemotherapy is a stressor that results in anxiety as one of the responses.

If one looks at only the means in this study, without statistical analysis, all subjects show a pattern of lower end measure means than baseline means for epinephrine, norepinephrine levels, state anxiety, and trait anxiety scores. That is to be expected because at the beginning all subjects were facing the unknown of chemotherapy, and at the end measurement they were at the end of the treatment. Means for norepinephrine appear lower in all measures for the experimental group, despite one experimental subject having the highest recorded baseline norepinephrine level of all subjects. 
A curious pattern seems to appear in the middle measure for the anxiety measurements. In the experimental group, a drop from baseline to Measure $\# 2$, the middle measurement, is seen with epinephrine and both state and trait anxiety levels means. At the third and final measure, the means go up from Measure \#2, but remain below baseline for epinephrine and state and trait anxiety, and drop further from baseline for epinephrine. This pattern is different in the control group, where means for epinephrine, state anxiety, and trait anxiety go up from baseline at measure \#2, while norepinephrine drops very slightly. At the final measure, control means for epinephrine are the same as at baseline, means for norepinephrine and state anxiety are lower than baseline, and trait anxiety is slightly higher than at baseline. None of these are statistically significant, due to the small sample size and the standard deviation in each group, but there is a pattern that is a positive indicator for further study with a larger population

With ANCOVA, norepinephrine levels show a trend toward being significantly lower in the experimental group as compared to the control group at the final measure. A greater decrease in levels in experimental subjects who practiced PMR can be seen when looking at groups according to PMR use. Norepinephrine levels drop in the experimental groups that had moderate PMR use and very slightly in the high PMR use group, while levels go up in the control group and also go up slightly in the no PMR use experimental group.

The state anxiety score is significantly lower at the second measure in the experimental group as compared to controls. Trait score means also appear lower at the second assessmentin the experimental group, compared with the control group, although the difference is not statistically significant. It would seem logical that persons with higher state or trait anxiety scores 
would have higher epinephrine levels. However, this pattern did not emerge in the means shown in Table 2, and at least at baseline the experimental group showed lower epinephrine levels and higher state and trait anxiety levels than did the controls.

Although interpretation of this requires a larger sample and further study, this pattern and subjective statements by subjects in the experimental group suggest that the most important difference between groups might be at the mid-point rather than at the beginning and at the end points of chemotherapy. It is possible that at baseline all subjects have similar anxiety levels because all are going to undergo chemotherapy and the process is an "unknown". At the final measure all subjects have completed three cycles of chemotherapy and are anticipating the final cycle and the end to the chemotherapy regimen. At this point all subjects, both experimental and control, expressed relief that the process was nearly over. At Measure \#2, however, the subjects had undergone two cycles of chemotherapy and were anticipating a third. It is possible that at this point the experimental subjects experienced a reduction in anxiety, whereas the control subjects may have either experienced no decrease or even an increase. This pattern seems to emerge in the means shown in Table 1 , and is validated by the subjective comments of subjects.

Interestingly, the experimental group actually had slightly higher state and trait means at baseline than the control group. This may be because one subject in the experimental group had a very high state score at baseline, the highest score possible, and a high score at Measure \#2. The subject was diagnosed with depression after three cycles of chemotherapy and started on treatment for depression at that time. At the fourth (final) cycle, and at the final data point, the diagnosis of depression and subsequent treatment was relayed to the researcher, but because the 
subject had completed the study, she was kept in with the rationale that she may very well reflect what one would encounter in clinical practice if enough patients were enrolled in a study.

When the PMR aspect is included, there is a downward directional trend to Measure \#2 and then an increase at Measure \#3 in both state and trait anxiety scores in the experimental group, regardless of how much PMR was practiced. The state anxiety scores dropped very slightly, in the control group, as compared to a larger drop in the experimental groups, and the trait score goes up. Variation in trait scores is not expected since trait anxiety level is expected to remain stable over time. As previously discussed, Taylor (1995) reported changes in trait anxiety following an intervention in HIV-positive individuals. It may be possible that life-changing events, such as diagnosis with cancer, result in a period when trait anxiety is not static, and this would be useful to study further in persons with cancer.

An interesting finding with the very small group involved was that the experimental group that did not practice PMR started out with the lowest trait anxiety score mean and showed the lowest end measurement on state and trait score means. This may because of personality characteristics that may have contributed to their not following the PMR regimen. It would be interesting to study whether this difference is associated with personality factors that reflect a more relaxed attitude in general on stress measurement instruments. Subjects who practiced high PMR use have higher norepinephrine level means both at baseline and end measurement, although they show a slight drop in norepinephrine compared to the increase in norepinephrine in the control group and the no PMR use group. The question arises whether the persons who practiced high PMR use may have had a more anxious personality than those who practiced moderate use. 
It would be interesting to study any correlation of a relaxation intervention and anxiety indicators including use of an anxiety inventory, serum catecholamines to assess for acute stress, and also cortisol levels to include stress of a more chronic nature.

Control subjects and experimental subjects who did not use PMR show an increase from baseline in norepinephrine level means, while experimental subjects who used moderate to high practice of PMR show a decrease from baseline. This raises the question of whether systematic use of PMR may result in modulation of stress hormone production for some persons, and would be of interest for further study.

The conceptual framework used for this research was the SCA framework, which includes the concepts of a stressor with resulting coping and adaptation. The intervention in the research was aimed at assisting the individual in the intervention group with both psychological and physiological coping with the stressor of the cancer treatment process, seeking a resultant adaptation showing lower catecholamine levels and state and trait anxiety score than the nonintervention group. It appears that the experimental group at mid-point had lower indicators of anxiety than did the control group, and it is possible that the intervention played a part in this psycho-physiological adaptation response to the stressor. This is consistent with previously reported research, which shows a decrease in anxiety scores associated with some types of relaxation intervention, such as that reported by Walker et al. (1999) who found that women who were receiving chemotherapy for breast cancer and were in a group that practiced relaxation training involving progressive muscle relaxation and guided imagery had less anxiety than women in a control group. 


\section{Nausea \& Emesis}

No significant difference in nausea and emesis was found between the control and experimental group. There are three likely reasons for this finding. First, patient report of anticipatory side effects increases with the number of cycles received. The regimen used in this study was a combination of $\mathrm{AC}$, which required only four cycles. No studies reporting ANV specific to the combination of $\mathrm{AC}$ were found reported in the literature. It may be that the duration of the therapy is too short for ANV to occur, and had the regimen required further cycles more ANV might have been reported. Second, the treatment regimen for all subjects included rigorous management of nausea and vomiting associated with the chemotherapy, and this could likely decrease report of and incidence of ANV. Third, the sample size is small; a larger sample would have been very helpful in giving a better picture of any association of ANV with AC chemotherapy.

For the purpose of further research, the aspect of anticipatory nausea and emesis, as measured by MANE, may not be a high priority for inclusion in a study, if chemotherapy of short duration is used and if rigorous anti-emetic management is a part of the treatment regimen. Of more significance would be use of an instrument evaluating the amount of distress associated with the symptom of chemotherapy-associated nausea. It is recommended that use of a symptomdistress scale designed for chemotherapy recipients be included in further research of this nature. Immunity

A major confounding variable in this study was that a number of subjects required the GCSF Neupogen when their granulocyte counts dropped. On the surface it appears that Neupogen 
was used in more of the control subjects than in the experimental group. Two of the seven experimental subjects (28\%) were treated with Neupogen, while three of the five control subjects (60\%) required the treatment. Although Chi-square analysis showed no significant difference, it would be of interest in a larger study of this nature to see if there were any difference between groups in the number of persons who required G-CSF. Use of G-CSF with chemotherapy is reported in the literature, but none was found that projects the percentage of persons undergoing AC chemotherapy or for that matter, any of the major chemotherapy regimens, who would be expected to require G-CSF. This complicates planning immune function studies in further similar research of persons undergoing chemotherapy, because the effect of the G-CSF renders the results unusable with results from persons who did not require G-CSF. It may be of more benefit to eliminate immune function tests and instead focus on the clinical criteria for G-CSF need.

No differences were seen between groups for IgG and IgA. At Measure \#3 the end measure, the experimental group had a significantly higher IgM than the control group. What this means is unclear and it would be helpful to evaluate with a larger sample.

No meaningful significant differences were found with cell subsets, mitogen stimulation, or NK activity. It was anticipated that if changes were to be seen, they would be associated with NK function. This is because decreased NK function has been found to correlate with a number of psychological factors such as elevated stress level (Anderson et al., 1998), loneliness (KiecoltGlaser et al., 1984), bereavement (Irwin et al, 1987a), depression (Andreoli et al., 1993), and lack of social support, depression, or fatigue (Levy et al., 1987). Certain life events, such as examination stress, have also been associated with decreased NK activity (Kiecolt-Glaser et al., 
1984). However, in contrast, Bachen et al. (1992) found in a group of healthy subjects exposed to an acute laboratory stress situation that there was an increase in the number of circulating NK cells. Levy et al. (1987), studying patients undergoing chemotherapy for breast cancer, found that decreases in NK activity was correlated with factors such as depression or lack of support, but not with the actual chemotherapy. It is hypothesized that certain relaxation interventions can enhance NK activity in persons who are experiencing stressful situations. Zachariae et al. (1990) found a significant increase in NK cell function after guided imagery practice in a healthy group of subjects. However, Kiecolt-Glaser et al. (1984) did not find that duration of relaxation practice, comprising self-hypnosis, progressive relaxation, imagery exercise, and/or autogenic training, affected NK activity in a study of healthy individuals. This study found no differences between groups in terms of NK numbers or activity in the small number of subjects with results that could be analyzed.

Lymphocyte response from mitogen stimulation is a way of assessing the functional aspect of the immune system. Mitogen stimulation with PHA alone would not be used again in further study unless a very good reason became apparent. Using mitogen stimulation with only PHA limits one to evaluating T-lymphocyte but not B-lymphocyte activity. The limited findings and difficulty in getting the test performed consistently in this setting does not justify the cost at this time.

Increases in cytokine activity can help the researcher understand the type of immune response being mobilized and what cells are probably most active (Vedhara et al., 1999). Cytokines may be responsible for activation of the cellular or humoral aspects of the immunity. 
Only a limited number of cytokines can be detected in plasma of healthy individuals, and according to Vedhara et al. (1999), their measurement is generally more useful in diseased populations. In this study, IL-2 (a Thl cytokine) levels were found at higher than reference range levels in the control group but not in the experimental group. Investigation of this finding revealed that only

one sample of the thirty analyzed showed any result, and there was strong evidence that the finding was due to error, so the results were considered negative and were not analyzed.

At Measure \#2 the individual in the control group had a significantly higher IL-6 level (a Th2 Cytokine) than did those in the experimental group $(t=11.602, p=.007)$. This could be a random event and really cannot be interpreted based on the sample size. If indeed it is a valid finding, it may reflect an up-regulation of the Th2 cytokine arm at this measurement. Upregulation of one has a suppressive effect on the other. One would expect to see a suppression of Th1 cytokines if there is an up-regulation of Th2 cytokines and vise-versa. In this study, one would expect to see up-regulation of Th2 cytokines (e.g. IL-4 and IL-6) if there is an downregulation of the Thl cytokines (e.g. IL-12). There seems to be a pattern of lower IL-12 means in the experimental group at Measurement $\# 2$ and baseline, but these do not approach statistical significance, and a larger sample is needed to evaluate the findings. It would be of interest in further study to evaluate the cytokines $\mathbb{I}-4, \mathbb{L}-6, \mathbb{L}-10$, and $\Pi-12$ to see if any significant trend is seen in persons undergoing chemotherapy.

There are some difficulties with evaluating the cytokine findings in this study because of some of the unknown information. First, it is difficult to know if the lack of results for IFN 
gamma and IL-2 are actual or instead because of sample decay during storage, even though the samples were maintained frozen at the appropriate temperature. It is likely that the findings reflect real results, but a larger sample would have helped clarify this. Second, it is most likely that the single IL-2 finding was due to a contaminant. However there is a very small chance that it was a real result. The IL-10 results were lost because of a manufacturer kit problem and would have been helpful in looking at the larger picture of TH1:TH2 balance had they been available. Six cytokines had been chosen to represent both Th1 and Th2 immune function. Only three of these showed growth, although the two that showed no growth could also be considered valid. The Thl cytokines, particularly Il-2, occur in very small amounts and are difficult to measure in plasma, particularly in healthy hosts. Persons undergoing chemotherapy are not usually considered healthy hosts, and it may be that chemotherapy affects detection of the cytokines used in this study. That information was not found reported in the literature about AC therapy. Theoretically one would like to study cytokines that would permit assessment of the Th1:Th2 balance. However, measurement of cytokines in plasma is difficult for a number of reasons. The amounts present for measurement are very small, and also it is uncertain how much of the cytokine is free and how much is receptor-bound. In considering further study, a design would probably not include cytokines IL-2 or IFN gamma because the expected level is zero in the non-diseased host. Use of a new method that involves assessing the messenger RNA for the cytokine would be explored instead. Third, G-CSF is a powerful cytokine and use in subjects would alter cytokine results. There may be other significant reasons for the limited statistically significant findings. These could possibly be related to the cytotoxicity of the chemotherapy agents. Despite careful 
searching, no literature was found reporting the effects of the AC combination chemotherapy on the immune function tests used in this study. There was literature discussing one or the other of the drugs, or other combinations of the drugs, but there appears to be a dearth of information specifically about AC. This complicated the process of choosing which immune function tests to use in the study.

One key aspect missing in this study was the subjective aspect of how persons undergoing chemotherapy felt about the process. Although there was no formal process for addressing the subjective aspect, there was an informal recording of some of the information. Six of seven experimental subjects volunteered that participation in the study, including the massage and interaction with the researcher, seemed to make the whole experience less negative. Four subjects used the words "I don't dread coming in for chemo" or "I don't dread coming in for chemo so much" in their volunteered statements. One subject stated that she "almost looked forward to coming in" when discussing coming in for chemotherapy treatment, and went on to say that it gave her an opportunity to talk to someone who would listen. None of the control subjects gave any positive expression about the chemotherapy duration until the end, when they made statements about being glad that it was over.

A curious subjective observation, made over time by the researcher, was that at the second assessment, most subjects seemed to express more negative feelings than at other times regarding the chemotherapy process and how they were feeling and coping with the side effects and changes associated with the treatment. Subjects appeared more relaxed and even happy at the final assessment because, as they expressed it, they knew it was the last AC treatment, and they could 
look forward to relief from some of the negative side effects associated with their therapy. This might explain why there seemed to be an increase at the second assessment in both state and trait anxiety scores in the control subjects. However, this trend of increased state and trait anxiety scores was not reflected in the experimental subjects, although because the researcher was more involved with them (giving the massage), they expressed more to the researcher about their negative feelings at the second assessment than did the control group. Further study would well include the subjective aspects of patient expressions. Some of these aspects might be addressed by using a quality of life inventory and a symptom distress scale, both quantitative, but patients should also be given the opportunity to express what they perceive as important subjective aspects.

\section{Proposed Plan for Further Study}

Based on the findings in this pilot study, a proposed plan for further study would include some changes. The design proposed would include a prospective two-group experimental intervention design. The intervention would include PMR and either a teaching support interaction or massage prior to each chemotherapy cycle. Assessments would include STAI-Y for anxiety as well as the stress hormones epinephrine and norepinephrine to assess for acute stress and cortisol to assess for chronic stress. Instead of MANE, a Symptoms Distress Inventory specific to cancer patients would be used, along with a Coping Inventory to assess how individuals were reacting to and coping with the chemotherapy. Very limited immune function tests would be proposed, but clinical information including use of G-CSF would be collected. Possibly tests to assess for NK numbers \& function several months after chemotherapy 
completion would be done. Tests reflecting the Th1:Th2 balance would be strongly considered; including possibly the cytokines $\mathbb{I L}-2, \mathbb{L}-4, \mathbb{I L}-6$, and $\mathbb{L}-10$, because these could be frozen and stored for processing at the end of the data collection and subjects requiring G-CSF could be removed from the data set. However, another strategy that would be explored would be tests to measure cytokine RNA messenger instead. The sample size would be increased, and although it would increase variability, it might include more than one specific type of chemotherapy, using categories of chemotherapy with similar side effects.

The overall goal of a future study would be to examine whether a nursing intervention could modify side effects of the psycho-biological stressor, chemotherapy, and enhance individual coping and adaptation in persons who require the treatment. 


\section{Chapter V}

Summary, Conclusions, and Recommendations

With the bi-directional interplay among the endocrine, neurological, psychological, and immune systems, it is clear that stressors can have psycho-biological responses that affect all of these systems. Chemotherapy, which large numbers of persons undergo, can be considered both a physiological and a psychological stressor. Chemotherapy has known side effects that may adversely affect the outcome or the process of treatment. Anxiety, nausea, emesis, and immunosuppression are commonly associated with chemotherapy. Anxiety can be associated with a number of negative side-effects, including nausea and emesis, anticipatory nausea, and even anticipatory immunosuppression. Some psychological stressors such as anxiety can result in down-regulation of certain immune functions. Many types of chemotherapy are also directly associated with immunosuppression. Some interventions have been found to modify stress-related immune modulation. This has led to questions about whether an intervention designed for relaxation could modify the stress response to chemotherapy resulting in a reduction in degree of side effects.

\section{Summary of Study Findings}

In this study, the experimental group demonstrated significantly less state anxiety at midmeasure than did the control group and showed a trend of lower norepinephrine level at the end measure. The findings tend to support the research hypothesis that a nursing relaxation intervention would result in lower anxiety in the research group as compared to controls in this study group. 
The findings on nausea and emesis did not support the research hypothesis; no difference was found between groups. This really was also true of the immune test findings, because although there were statistically significant differences or trends with four measures, these are meaningless because of the small sample size.

The goal of obtaining information of the effect of Adriamycin/Cytoxan chemotherapy on the immune system was met in this pilot study. It became apparent that this regimen has a profound effect on immune function, requiring, in a number of cases, treatment with G-CSF in order to continue chemotherapy. Although the immunosuppressive effects of this regimen are known and expected, literature on the specific effects of AC chemotherapy on the immune function tests used in this research, and the expected incidence of G-CSF requirement, were not found in literature searches. This pilot study has provided a basis for omitting these tests in further planned study of this nature. The results of immune function tests for persons who received GCSF may be evaluated at a later date and compared with those who did not receive G-CSF to see if that information might be useful to oncology clinicians since nothing of that nature was found in the current medical or cancer literature. A plan for further study did emerge with a number of changes from the pilot study.

Methodological Limitations

There were a number of methodological limitations involved in the study. Some of these reflected the nature of clinical research where there are variables in terms of timing, blood cell counts, and other uncontrollable factors. 
States of health differ between participants and could account for differences seen in the study. There may have been unidentified underlying health problems that could have influenced the study. Variations of hormone and immune levels may occur based on individual differences, circadian rhythms, and circumstances of daily life (e.g., no sleep).

Stressors, both positive and negative, are a part of life and cannot be eliminated. When studying a specific stressor, such as the chemotherapy experience, it is impossible to separate stress reactions associated with the chemotherapy completely from stress reactions associated with other stressors occurring at the same time. Subjects in both the experimental and control groups of the study had significant stressors in their lives. One subject had just started a job after a period of unemployment. She had minimal health insurance and had to work during the whole course of chemotherapy. Her work was based on commission, and she had no savings to fall back on. Her elderly father who lived some distance from her was very ill, and her mother was unable to manage adequately, so they relied on the subject for help. Another subject not only had a disabled child who required major surgery during the chemotherapy regimen, but also discovered that her father had a rather large bowel cancer and required treatment. Another had, in addition to several other children, a severely mentally handicapped child who required considerable and constant care. Although not all had stressors to the same extent, all of the subjects reported feelings of stress related to the diagnosis, the treatment, and side effects such as the loss of hair, and concern about the outcome, in addition to concerns about keeping up with normal daily activities. 
Another potential limitation was that individuals differ in response to interventions such as massage or progressive muscle relaxation. If changes occur, one cannot assume that the change is due to intervention alone. In addition, while subjects were asked to practice PMR while at home, there was no way to assure that subjects would not only do so, but would also report their activity accurately. It was important to the study that the practice of PMR not be an additional negative stressor to the subjects. Two subjects did not practice it after the first few times. One of them volunteered to drop out of the study but then elected to stay in. Another subject did not bring in the cards noting practice, even when reminded by telephone. She changed the subject consistently when asked about how often she was able to practice, even when assured that there was no negative aspect with number of times practiced, and it was only an attempt to see if there was any relationship with number of practices and any outcomes. Of the subjects who filled out the cards and returned them, the times of practice ranged from daily to sporadically.

Self-reported subjective phenomena such as anxiety and nausea may be difficult to discriminate between (Andrykowski, 1990), and they may influence how subjects responded to the STAI and the MANE. In addition, venipuncture (e.g., for blood samples) may increase aversiveness and anxiety (Andrykowski, 1990) of subjects and may be reflected in self-reporting of anxiety.

An unforseen limitation involved the types of chemotherapy regimens offered patients. The study was developed initially, in conjunction with the chief of Oncology, to include patients scheduled for CMF (Cyclophosphamide, Methotrexate, and 5-FU) chemotherapy. It was anticipated that between six and eight patients per month would be treated with this combination 
and so would be available as a potential subject pool. After all the appropriate approvals had been obtained, the researcher met with the contact physician in the Oncology department to proceed with data collection, and at that time found out that there had been some major changes in the department, among them that there was a new chief of the department and, the oncology center had joined a national cancer study. The drug protocols now anticipated include a number of combinations to meet the national study requirements, none of which included CMF therapy. The researcher then met with the new chief of the Oncology department and redesigned the study to use patients with breast cancer who were in a protocol that required four doses of $\mathrm{AC}$ chemotherapy. Some of these patients would go on to get additional drugs (e.g., Taxotere or Tamoxifen), but all would initially get $\mathrm{AC}$ for four doses. The study proposal and consent forms were rewritten and re-submitted to appropriate review committees, and once approval was obtained again, data collection began. Patients with breast cancer who were treated at the Oncology department were randomly assigned to one of several national chemotherapy protocols, only one of which involved AC alone. This resulted in the number of potential subjects dropping from an anticipated six to eight per month with the originally designed study using CMF to an anticipated zero to two per month. During one period, there were none that met the criteria for nine months. This was an unforseen, uncontrollable, and rather frustrating aspect that limited the number of potential subjects available.

The change in study design affected assessment of conditioned nausea and vomiting. If a person is to develop ANV, it usually occurs after five to eight treatments. However, with the change to $\mathrm{AC}$ chemotherapy, only four doses were required, making it far less likely that patients 
would develop ANV. Another very positive aspect that affects ANV is that now rigorous antiemetic medication management is used to prevent and manage nausea and emesis, so the problem is not as extensive as it once was.

Another limitation had to do with timing of clinic visits. At the beginning of the study patients would come to the clinic for routine blood work and to see the physician. Their chemotherapy would then be given the following day. This gave two opportunities for the researcher to establish contact with subjects in the clinic setting. After the first year this changed so that the patient would get the blood work, see the physician, and receive chemotherapy on the same day if the blood counts were satisfactory. The researcher then made arrangements to come to the clinic when a subject was scheduled to come in. In addition, a note to page the researcher was attached to clinic records, and either the receptionist at the front desk or an oncology nurse received the message and request to page. Initially subjects were also asked to call if any changes in their schedule occurred. However, because chemotherapy was dependent on blood counts, subjects did not know if there would be a change in dose schedule until the time of the visit. It became apparent that asking subjects to contact the researcher was a negative stressor. Therefore, the researcher took responsibility for assuring follow-up when the subjects came to the clinic. For the most part this worked well. However, there were several occurrences where the researcher had personally come to the clinic when a subject was scheduled and spoken with staff and left written request to be contacted when the subject actually came in, but because of an extremely busy flow of patients, the contact did not occur. Twice the researcher was paged only after chemotherapy was actually started, so the data collection point was lost. This was understandable 
though, because the clinic was very busy and crowded, and staff were under a great deal of pressure to prepare chemotherapy drugs and administer them under time, space, and distraction constraints. Their first concern was for care and safety of the patient, but it did affect data collection.

The process of blood collection for specimens in the outpatient laboratory setting was also challenging. Patients who did not have a central venous access device (e.g., a port-a-cath) had blood drawn in one of two laboratories in the clinic. Detailed arrangements were made with hoth laboratories. A small bag containing appropriate collection tubes and instructions was provided, and personal contact was made just before the subject was to come in for blood draw. Subjects were called the day before to find out when they were planning to come in, and a note requesting that the researcher be paged and the time the patient was coming in was placed with their name at the desk where they would check in for blood-draw. The researcher tried to come to the laboratory when the patient was scheduled to come in, but this was not always possible because the clinic site was at another part of the university campus from the researcher's work site, and often patients would be quite late for appointments, necessitating long waits for them to arrive. Blood collection went flawlessly in one of the laboratories, but was problematic in the other. On one occasion, a subject in the study reminded the person drawing blood that the research specimen was to be drawn, and was assured that it had been obtained. However it had not, and follow-up revealed that the venipuncutrist was simply trying to placate the subject. On two other occasions, the request to page the researcher was not followed. There was a "double-check" on this problem by having the clinic staff page the researcher before chemotherapy was instituted, so 
that if a specimen was missed in the clinical laboratory, it could be drawn when venous access was established for chemotherapy, and on one occasion the specimen was obtained at that time. The only drawback to this was that the oncology nurses could not wait more than a few minutes for the researcher to arrive because of back-up of patients waiting for chemotherapy, and on more than one occasion started chemotherapy without paging the researcher. The agreement was that the study would not interfere with normal chemotherapy administration, so this was understandable. However, these occurrences resulted in missed measures, which complicated the initial problem of having a small sample size.

Another complication occurred in the main laboratory that processed the specimens. All specimens were transported according to the required protocol, in correct tubes, labeled, and with the corresponding order slip and also instructions about the study and to call the researcher if there were any questions. About $20 \%$ of the specimens taken to one of the laboratories were mishandled once there, resulting in missing data. A great deal of attention was paid to this problem once it was identified, but the outcome was that a lot of data were missing from specimens processed by this laboratory. All of the specimens that were taken to the second laboratory, an approved designated research laboratory for immune studies, were processed properly. There the only problem was with timing of specimens for mitogen response. If the specimens were submitted on a Wednesday or a Thursday, they could not always be processed because that laboratory was a research laboratory with staff who were not routinely scheduled to work on the weekend, and these specimens required handling at 48 and 72 hours. If there was a diagnostic patient test in process that required management over the weekend staff were 
scheduled to be in the laboratory and processed the research mitogen response tests along with the diagnostic patient tests. Thus some of the mitogens were missing from samples submitted to this laboratory.

Occasionally an unavoidable problem occurred when there was not enough blood sample to complete the panel of tests. There were a few tests reported as "not enough cells for processing".

Midway through the data collection, there was a change in the amount of blood required by the laboratory for catecholamine processing, going from a requirement of $5 \mathrm{ml}$ to $10 \mathrm{ml}$. This information was not relayed to the researcher, despite a written request with each sample to call a telephone or pager number in case of questions, and was discovered only when some specimens were simply reported as "not processed." Once the requirement was noted by the researcher, the additional amount of blood was collected. Initially a total of $25 \mathrm{ml}$ of blood was obtained, with a $10-\mathrm{ml}$ tube and three $5-\mathrm{ml}$ tubes used. When the requirement was raised to $30 \mathrm{ml}$, it required two 10-ml tubes and two 5-ml tubes. Even though it was explained as "two tablespoons of blood" the sight of the tubes had an initial negative psychological impact on the subjects. Earlier subjects did not comment very much on the $25 \mathrm{ml}$ draw, simply making a few jokes about the amount of blood drawing in general. However, when the change was made, subjects focused on the two large tubes and expressed some concern about the amount of blood loss they were experiencing. They were reassured that the amount, in addition to their clinical needs, was within established guidelines of safety according to the protection of human subjects committee. Two subjects, 
however, refused to participate in the study when they saw the collection tubes set out for the blood sample.

There was also considerable variation among experimental subjects in practice of PMR. One participant openly stated, at the second assessment, that she was unable to fit the PMR practice into her day. She had already been started in the experimental group and was kept in the study. One participant simply refused to provide the card enumerating the number of practices, and with careful and diplomatic questioning, it became apparent that she was not doing the PMR. Final evaluation revealed that two of the experimental subjects did not practice PMR at all after the first two or three times and fit a category of no practice (no PMR).Two subjects practiced PMR between $10-50 \%$ of the time and were considered in a category of moderate use (moderate PMR), and three of the subjects practiced PMR at least $50 \%$ of the time and up to about $90 \%$ of the time and fit a category of high use (high PMR). Assessment measures were run in the form of a descriptive graph to show if there was any trend of difference in test results among subjects practicing PMR at different degrees. In general, those who did not practice PMR were more similar to the control group than to those who made moderate to high use of PMR. The numbers involved were too small to be of statistical significance, but they were displayed in descriptive graphs showing the differences between the control group and the experimental group broken into three categories according to how much PMR was practiced.

A final limitation was related to the need for use of a granulocyte colony stimulating factor (G-CSF) in subjects whose blood counts dropped dangerously. G-CSF directly affects the immune system since it is a cytokine that stimulates production of granulocytes. Two of the 
experimental subjects $(28.5 \%)$ and three of the control subjects $(60 \%)$ required use of G-CSF when their granulocyte counts dropped. In the past these subjects would have required blood transfusion, but development of G-CSF has been of real benefit to these subjects, and the researcher was aware that some subjects might require it. Use of G-CSF was recorded in data collection. It was unknown at the start how this would affect the study results, because the effect of G-CSF on immune function tests in research of this nature had not yet been reported, and there was no way of anticipating who would require G-CSF.

\section{Recommendations For Nursing Practice}

These findings have implications for nurses who are evaluating and designing management strategies for clients who are facing a stressor such as chemotherapy. In this very small population, state anxiety was lower at one measurement during the process of chemotherapy in the group that underwent relaxation intervention. If the findings can be validated in further study, it may have significant implications for treatment of cancer patients. Relaxation interventions can be inexpensive and can be used widely and independently. If the findings can be generalized to a larger population with further study, relaxation interventions could be planned into the treatment regimen of chemotherapy clients. Promoting these strategies could not only enhance response to therapy but also improve quality of life in patients requiring chemotherapy.

Taking care of the person as a whole is a common concern of nurses in this era of health management and increasing technology. This requires individualized assessment and planning of interventions that meet the person's needs. Relaxation interventions are one way of addressing the individuals needs in response to stressors, with the goal of helping them adapt in both 
psychological and physiological ways that promote health. This is also a timely topic with the current increased awareness and interest in complementary therapies. With studies that are supportive of such therapies, some health maintenance organizations (HMOs) are now reimbursing for selected relaxation interventions for patients undergoing chemotherapy because the outcome seems to be to the advantage of both the patient and the HMO. This is a time when nursing validation of the effectiveness of these therapies could affect payment for them.

\section{Recommendations for Nursing Education}

This pilot study indicates that relaxation might result from a nursing intervention. However, extensive review of the literature to support the study revealed that few nurses are involved in or publishing in the area of neuro-immunology. A recommendation for nursing education is to place more emphasis on physiological and research aspects in the curriculum to provide a basis or interest in psycho-biological intervention research. Nursing education could address the aspect of psychoneuroimmunology in terms of how body systems interact and respond to psychological and biological stressors. At this time, most new nursing physiology texts address this aspect briefly, but it is not a strong component in nursing education. In addition, more credibility could be given to relaxation interventions in nursing curricula. It is not infrequent to hear persons who have recently been hospitalized complain that they never received a back massage. Relaxation interventions such as back massage have given way to use of analgesics and the nursing complaint that there is no time for interventions like massage. Nurses need to be educated about the underlying physiological and psychological benefit that can result with these simple and relatively inexpensive interventions. They need to learn which interventions are best 
suited for various situations, and how to evaluate outcomes for these interventions so that their use can be justified as a necessary part of nursing care.

\section{Recommendations for Further Research}

Additional research is needed to explore further whether nursing intervention such as specific relaxation strategies can result in modification of side effects associated with psychobiological stressors such as chemotherapy. Ideally a much larger sample size should be used. Studies of this nature tend to have small sample sizes because of the very high cost involved with the laboratory tests. This contributes to lack of "statistical significance". However, if upon replication one consistently gets the same pattern, the "statistical significance" per se may not be the critical factor. Similar findings over time and repeated studies could indicate significance even if the individual study does not show statistical significance.

Another aspect that would be very helpful for future research would be to study what changes are associated with specific interventions, and also whether the degree of use of the interventions makes a difference. Further research could study whether there was a difference in findings based on how often subjects practiced PMR. Additionally, research is needed on personality types and who is most likely to benefit from certain types of intervention. This pilot study raises the question of whether personality factors were associated with persons who did not practice PMR, compared with persons who had moderate or high use of PMR, and it leads to the question of whether personality aspects are correlated with anxiety levels and stress hormone levels.

Further research also needs to evaluate the effect of G-CSF on immune measures in persons undergoing chemotherapy. The effect of G-CSF on research of this nature has yet to be 
reported and may be an area for collaborative research. Another potential area for collaborative research is in defining clearly the immunological changes that occur with Adriamycin and Cytoxan chemotherapy. Although parts of this information are available in various sources, none was found that described the effects of this combination.

There is also a need for a qualitative aspect to this type of study. In the experimental group, five stated that they did not "dread" coming to the clinic for their treatments and one stated that she "almost looked forward to chemotherapy" because of the intervention and resultant interaction with the researcher. This information was unsolicited and offered voluntarily by the subjects. None of the control group offered similar comments. It would be helpful to include a qualitative aspect in future research to enrich and possibly explain in another way the empirical findings.

\section{Conclusion}

Chemotherapy is widely used and has significant side effects. It is anticipated that further study may result in methods of management that can modify the degree of the side effects and enhance patient quality of life and response to therapy. Although the findings of this study have been limited there seems to be evidence that further study is warranted, and the data collected will be useful in designing further study. Despite the challenges inherent in clinical research, it is important that further clinical studies be undertaken. 


\section{References}

Aapro, M. S., Kirchner, V., \& Terrey, J. P. (1994). The incidence of anticipatory nausea and vomiting after repeat cycle chemotherapy: The effect of granisetron. British Joumal of Cancer, 69, 957-960.

Ader, R., \& Cohen, N. (1975). Behaviorally conditioned immunosuppression. Psychosomatic Medicine, 37, 333-340.

Ader, R. \& Cohen, N. (1991). The influence of conditioning on immune responses. In R. Ader, D. L. Felten, \& N. Cohen, (Eds.), Psychoneuroimmunology, (2nd ed).(pp. 611-646). New York: Academic Press, Inc.

Ader, R., \& Cohen, N. (1993). Psychoneuroimmunology: Conditioning and stress. Annual Review of Psychology, 44,53-85.

Ader, R., Cohen, N., \& Felten, D. (1995). Psychoneuroimmunology: Interactions between the nervous system and the immune system. Lancet, 345(8942), 99-103.

Ader, R., Felten, D., \& Cohen, N. (1990). Interactions between the brain and the immune system. Annual Review of Pharmacological Toxicology, 30, 561-602.

Ader, R., Kelly, K., Moynihan, J. A., Grota, L. J., \& Cohen, N. (1993). Conditioned enhancement of antibody production using antigen as the unconditioned stimulus. Brain, Behavior, and Immunity, 7, 334-343.

Adler, N. (1994). Health psychology: Why do some people get sick and some stay well? Annual Review of Psychology, 45, 229-259. 
Anderson, B. L., Farrar, W. B., Golden-Kreutz, D., Kutz, L. A., MacCallum, R., Courtney, M. E., \& Glaser, R. (1998). Stress and immune responses after surgical treatment for regional breast cancer. Journal of the National Cancer Institute, 90, 30-36.

Andreoli, A. V., Keller, S. E., Rabaeus, M., Marin, P., Bartlett, J. A., \& Taban, C. (1993). Depression and immunity: Age, severity, and clinical course. Brain, Behavior, and Immunity, 7 , 279-292.

Andrykowski, M. A. (1988). Defining anticipatory nausea and vomiting: Differences among cancer chemotherapy patients who report pretreatment nausea. Journal of Behavioral Medicine, 11, 59-69.

Andrykowski, M. A. (1990). The role of anxiety in the development of anticipatory nausea in cancer chemotherapy: A review and synthesis. Psychosomatic Medicine, 52, 458-475. Andrykowski, M. A., Jacobsen, P. B., Marks, E., Gorfinkle, K., Hakes, T. B., Kaufman, R. J., Currie, V. E., Holland, J. C., \& Redd, W. H. (1988). Prevalence, predictors, and course of anticipatory nausea in women receiving adjuvant chemotherapy for breast cancer. Cancer, 62 , 2607-2613.

Andrykowski, M. A. \& Redd, W. H. (1987). Longitudinal analysis of the development of anticipatory nausea. Journal of Consulting and Clinical Psychology, 55, 36-41.

Andrykowski, M. A., Redd, W. H., \& Hatfield. A. K. (1985). Development of anticipatory nausea: A prospective analysis. Journal of Consulting and Clinical Psychology, 53, 447-454.

Arakawa, S. (1995). Use of relaxation to reduce side effects of chemotherapy in Japanese patients. Cancer Nursing, 18, 60-66. 
Bachen, E. A., Manuck, S. B., Marsland, A. L., Cohen, S., Malkoff, S. B., \& Rabin, B. S. (1992). Lymphocyte subset and cellular immune responses to a brief experimental stressor. Psychosomatic Medicine, 54, 673-679.

Bauer, W. C., \& Dracup, K. A. (1987). Physiological effects of back massage in patients with acute myocardial infarction. Focus on Critical Care, 14, 42-46.

Beeken, J. E., Parks, D., Cory, J., \& Montopoli, G. (1998) The effectiveness of neuromuscular release massage therapy in five individuals with chronic obstructive lung disease. Clinical Nursing Research, 7, 309-325.

Bellinger, D. L., Madden, K. S., Felten, S. Y., \& Felten, D. L. (1994). Neural and endocrine links between the brain and the immune system. In C. E. Lewis, C. O'Sullivan, \& J. Barraclough, (Eds.), The psychoimmunology of cancer. Mind and body in the fight for survival. (pp. 55-106). Oxford: Oxford University Press.

Benschop, R. J., Jabaaij, L., Oostveen, F. G., Vingerhoets, J. M., Kirschbaum, C., Duivenvoorden, H. J., \& Ballieuz, R. E. (1993). Psycho biological factors related to human natural killer cell activity and hormonal modulation of NK cells in vitro. Life Sciences, 5, 18251834.

Berk, L. S., Tan, S. A., Fry, W. F., Napier, B. J., Lee, J. W., Hubbard, R. W., Lewis, J. E., \& Eby, W. C. (1989). Neuroendocrine and stress hormone changes during mirthful laughter. The American Journal of the Medical Sciences, 298, 390-396.

Berk, L. S., Tan, S. A., Napier, B. J., \& Eby, W. C. (1989). Eustress of mirthful laughter modifies natural killer cell activity. Clinical Research, 37, 989. 
Berk, L. S., Tan, S. A., Nehlsen-Cannarella, S., Napier, B. J., Lewis, J. E., Lee, J. W, \& Eby, W. C. (1988). Humor associated laughter decreases cortisol and increases spontaneous lymphocyte blastogenesis. Clinical Research, 36, 435A.

Bindemann, S., Soukop, M., \& Kaye, S. B. (1991). Randomised controlled study of relaxation training. European Journal of Cancer, 27, 170-171.

Blalock, J. E. (1989). A molecular basis for bidirectional communication between the immune and neuroendocrine systems. Physiological Reviews, 69, 1-31.

Blalock, J. E. (1994). The immune system. Our sixth sense. The Immunologist, 2, 8-15. Bottomley, A. (1997). Psychosocial problems in cancer care: a brief review of common problems. Journal of Psychiatric and Mental Health Nursing. 4, 323-331.

Bovbjerg, D. H. (1991). Psychoneuroimmunology implications for oncology? Cancer, 67, 828-832.

Bovbjerg, D., Cohen, N., \& Ader, R. (1987). Behaviorally conditioned enhancement of delayed-type hypersensitivity in the mouse. Brain, Behavior, and Immunity, 1, 64-71.

Bovbjerg, D. H., Redd, W. H., Jacobsen, P. B., Manne, S. L., Taylor, K. L., Surbone, A., Crown, J. P., Norton, L., Gilewski, T. A., Hudis, C. A., Reichman, B. S., Kaufman, R. J., Currie, V. E., \& Hakes, T. B. (1992). An experimental analysis of classically conditioned nausea during cancer chemotherapy. Psychosomatic Medicine, 54, 623-637.

Bovbjerg, D. H., Redd, W. H., Maier, L. A., Holland, J. C., Lesko, L. M., Niedzwiecki, D., Rubin, S. E., \& Hakes, T. B. (1990). Anticipatory immune suppression in women receiving cyclic chemotherapy for ovarian cancer. Journal of Consulting and Clinical Psychology, 58, 153157. 
Bridge, L. R., Benson, P., Pietroni, P. C., \& Priest, R. G. (1988). Relaxation and imagery in the treatment of breast cancer. British Medical Journal, 297, 1169-1172.

Bryla, C.M. (1996). The relationship between stress and the development of breast cancer: A literature review. Oncology Nursing Forum, 23, 441-448.

Burish, T. G., \& Carey, M. P. (1986). Conditioned aversive responses in cancer chemotherapy patients: theoretical and developmental analysis. Journal of Consulting and Clinical Psychology, 54, 593-600.

Burish, T. G., Carey, M. P., Krozely, M. G., \& Greco, F. A. (1987). Conditioned side effects induced by cancer chemotherapy: prevention through behavioral treatment. Journal of Consulting and Clinical Psychology, 55, 42-48.

Burish, T. G., \& Jenkins, R .A. (1992). Effectiveness of biofeedback and relaxation training in reducing the side effects of cancer chemotherapy. Health Psychology, 11, 17-23.

Burns, N., \& Grove, S. K. (1997). The practice of nursing research. Conduct, critique, and utilization. Philadelphia: W. B. Saunders Company.

Carnricke, C. L. M., Brantley, P. J., Bruce, B., Faruqui, S., Gresham, F. M., Buss, R. R., \& Cocke, T. B. (1988). Test-retest reliability and concurrent validity of the Morrow assessment of nausea and emesis (MANE) for the assessment of cancer chemotherapy-related nausea and vomiting. Journal of Psychopathology and Behavioral Assessment, 10, 107-116.

Carter, S. K., Bakowski, M. T., \& Hellmann, K. (1987). Chemotherapy of cancer, (3rd ed.). New York: John Wiley \& Sons. 
Carey, M. P., \& Burish, T. G. (1987). Providing relaxation training to cancer chemotherapy patients: a comparison of three delivery techniques. Journal of Consulting and Clinical Psychology, 55, 732-737.

Challis, G. B., \& Stam, H. J. (1992). A longitudinal study of the development of anticipatory nausea and vomiting in cancer chemotherapy patients: the role of absorption and autonomic perception. Health Psychology, 11, 181-189.

Clerici, M., Shearer, G.M., \& Clerici, E. (1998). Cytokine dysregulation in invasive cervical carcinoma and other human neoplasias: Time to consider the TH1/TH2 paradigm. Journal of the National Cancer Institute, 90, 261-263.

Cohen, S. (1995). Psychological stress and susceptibility to upper respiratory infections. American Journal of Respiratory Critical Care Medicine, 152, 553-558.

Cohen, R. E., Blanchard, E. B., Ruckdeschel, J. C., \& Smolen, R. C. (1986). Prevalence and correlates of post-treatment and anticipatory nausea and vomiting in cancer chemotherapy. Journal of Psychosomatic Research, 30, 643-654.

Cohen, S., \& Herbert, T. B. (1996). Health psychology: Physiological factors and physical disease from the perspective of human Psychoneuroimmunology. Annual Review of Psychology, 47, $113-142$.

Cohen, S., \& Rabin, B.S. (1998). Psychological stress, immunity, and cancer. Journal of the National Cancer Institute, 90, 3-4.

Cohen, S., Tyrrell, A. J., \& Smith, A. P. (1991). Psychological stress and susceptibility to the common cold. The New England Journal of Medicine, 325, 606-612. 
Coons, H. L., Leventhal, H., Nerenz, D. R., Love, R. R., \& Larson, S. (1987).

Anticipatory nausea and emotional distress in patients receiving Cisplatin-based chemotherapy. Oncology Nursing Forum, 14, 31-35.

Corley, M. C., Ferriter, J., Zeh, J., \& Gifford, C. (1995). Physiological and psychological effects of back rubs. Applied Nursing Research, 8, 39-42.

Cotanch, P., \& Strom, S. (1987). Progressive muscle relaxation as antiemetic therapy for cancer patients. Oncology Nursing Forum, 14, 35-37.

Cupps, T. R. \& Fauci, A. S. (1982). Corticosteroid-mediated immunoregulation in man. Immunological Review, 65, 133-154.

Dhabhar, F. S., Miller, A. H., McEwen, B. S., \& Spencer, R. L. (1995). Effects of stress on immune cell distribution. Dynamics and hormonal mechanisms. The Journal of Immunology, $154,5511-5527$.

Dillon, K. M., Minchoff, B., \& Baker, K. H. (1985-86). Positive emotional states and enhancement of the immune system. International Journal of Psychiatry in Medicine, 15, 13-17.

Eller, L. S. (1999). Guided imagery interventions for symptom management. Annual Review of Nursing Research, $12,57-84$.

Fakouri, C., \& Jones, P. (1987). Relaxation rx: Slow stroke back rub. Journal of Gerontological Nursing, 13, 32-35.

Fallowfield, L. J. (1992). Behavioral interventions and psychological aspects of care during chemotherapy. European Journal of Cancer, 28A(Suppl. 1), 539-541. 
Fawzy, F. I., Fawzy, N. W., \& Hyun, C. S. (1994). Short-term psychiatric intervention for patients with malignant melanoma: effects on psychological state, coping, and the immune system. In C. E. Lewis, C. O'Sullivan, \& J. Barraclough, (Eds.), The psychoimmunology of cancer. Mind and body in the fight for survival? (pp. 291-319). Oxford: Oxford University Press.

Fawzy, I. F., Kemeny, M. E., Fawzy, N. W., Elashoff, R., Mortin, D., Cousins, N., \& Fahey, J. L. (1990). A structured psychiatric intervention for cancer patients II. Changes over time in immunological measures. Archives of General Psychiatry, 47, 729-735.

Ferrell-Torry, A. T., \& Glick, O. J. (1993). The use of therapeutic massage as a nursing intervention to modify anxiety and the perception of cancer pain. Cancer Nursing, 16, 93-101.

Field, T., Grizzle, N., Scalfidi, F., \& Schanberg, S. (1996). Massage and relaxation therapies' effect on depressed adolescent mothers. ADOLESCENCE, 31, 903-911.

Field, T., Henteleff, T., Hernandez-Reif, M., Martineux, E., Mavunda, K., Kuhn, C., \& Schanberg, S. (1998). Children with asthma have improved pulmonary functions after massage therapy. The Joumal of Pediatrics, 132 854-858.

Fischer, D. S., Knobf, M. T., \& Durivage, H. J. (1993). The Cancer chemotherapy handbook. (4th ed.). St. Louis: Mosby.

Fittischen, B., Schultz, K-H, Schultz, H., Raedler, A., \& v. Kerekjarto, M. (1990). Changes of immunological parameters in healthy subjects under examination stress. International Journal of Neuroscience, 51, 241-242.

Frankenhauser, M. (1971). Behavior and circulating catecholamine. Brain Research, 31, $241-262$. 
Fraser, J., \& Kerr, J. R. (1993). Psychophysiological effects of back massage on elderly institutionalized patients. Journal of Advanced Nursing, 18, 238-245.

Fredrikson, M., Furst, C. J., Lekander, M., Rotstein, S., \& Blomgren, H. (1993). Trait anxiety and anticipatory immune reactions in women receiving adjuvant chemotherapy for breast cancer. Brain, Behavior, and Immunity, 7, 79-90.

Fry, W. F. (1994). The biology of humor. Humor, 7, 111-126.

Giraldi, T., Perissin, L., Zorzet, S., \& Rapozzi, V. (1992). Metastasis and neuroendocrine system in stressed mice. Annuals of the New York Academy of Sciences, 650, 297-301.

Glaser, R., Rice, J., Sheridan, J., Fertel, R., Stout, J., Speicher, C., Pinsky, D., Kotur, M., Post, A., Beck, M., \& Kiecolt-Glaser, J. (1987). Stress-related immune suppression: Health implications. Brain, Behavior, and Immunity, 1, 7-20.

Green, M. L., Green, R. G., \& Santoro, W. (1988). Daily relaxation modifies serum and salivary immunoglobulins and psychophysiologic symptom severity. Biofeedback and Self-Regulation, 13, 187-199.

Green, R. G. \& Green, M. L. (1987). Relaxation increases salivary immunoglobulin A' Psychological Reports, 61, 623-629.

Greenlee, R. T., Murray, T., Bolden, S., \& Wingo, P.A. (1999). Cancer statistics 2000. CA. A Cancer Journal for Clinicians, 49, 7-11.

Grochowicz, P. M., Schedlowski, M., Husband, A. J., King, M. G., Hibbard, A. D., \& Bowen, K. M. (1991). Behavioral conditioning prolongs heart allograft survival in rats. Brain. Behavior, and immunity, $5,349-356$. 
Groer, M., Mozingo, J., Droppleman, P., Davis, M., Jolly M. L., Boynton, M., Davis, K., \& Kay, S. (1994). Measures of salivary secretory immunoglobulin A and state anxiety after a nursing back rub. Applied Nursing Research, 7, 2-6.

Halvorsen, R., \& Vassend, O. (1987). Effects of examination stress on some cellular immunity functions. Journal of Psychosomatic Research, 31, 697-701.

Henry, J. P. (1987). The arousal of emotions: hormones, behavior, and health. Advances, Institute for the Advancement of Health, 6, 59-62.

Herbert, T. B., \& Cohen, S. (1993a). Stress and immunity in humans: a meta-analytic review. Psychosomatic Medicine, 55, 364-379.

Herbert, T. B., \& Cohen, S. (1993b). Depression and immunity: a meta-analytic review. Psychological Bulletin, 113, 472-486.

Herr, K. A., \& Mobily, P. R. (1992). Interventions related to pain. Nursing Clinics of North America, 27, 347-369.

Holland, J. C., Morrow, G. R., Schmale, A., Derogatis, L., Stefanek, M., Berenson, S., Carpenter, P. J., Breitbart, W., \& Feldstein, M. (1991). A randomized clinical trial of Alprazolam versus progressive muscle relaxation in cancer patients with anxiety and depressive symptoms. Journal of Clinical Oncology, 9, 1004-1011.

Houldin, A. D., McCorkle, R., \& Lowery, B. J. (1993). Relaxation training and psychoimmunological status of bereaved spouses. Cancer Nursing, 16, 47-52.

Ingle, R. J., Burish, T. G., \& Wallston, K. A. (1984). Conditionability of cancer chemotherapy patients. Oncology Nursing Forum, 11, 97-102. 
Irwin, M., Costlow, C., Williams, H., Artin, K. H., Chan, C. Y., Stinson, D. L., Levin, M., J., Hayward, A. R., \& Oxamn, M. N. (1998). Cellular immunity to Varicella-Zoster virus in patients with major depression. The Journal of Infections Diseases, 178(Suppl 1), S104-108.

Irwin, M., Daniels, M., Bloom, E. T., Smith, T. L., \& Weiner, H. (1987a). Impaired natural killer cell activity during bereavement. Brain, Behavior, and Immunity, 1, 98-104.

Irwin, M., Daniels, M., Bloom, E. T., Smith, T. L., \& Weiner, H. (1987b). Life events, depressive symptoms, and immune function. American Journal of Psychiatry, 144, 437-441.

Jacobsen, P. B., Bovbjerg, D. H., \& Redd, W. H. (1993). Anticipatory anxiety in women receiving chemotherapy for breast cancer. Health Psychology, 12, 469-475.

Jacobsen, P. B., \& Redd, W. H. (1988). The development and management of chemotherapy-related anticipatory nausea and vomiting. Cancer Investigation, 6, 329-336.

Janeway, C.A., \& Travers, P. (1997). Immunobiology. The immune system in health and disease. (3rd ed.). New York: Garland publishing Inc.

Jemmott, J. B., \& McClelland, D. C. (1989). Secretory IgA as a measure of resistance to infectious disease: comments on Stone, Cox, Valdimarsdottir, and Neale. Behavioral Medicine, $\underline{15}, 63-71$.

Kathol, R. G., Anton, R., Noyes, R. \& Gehris, T. (1989). Direct comparison of urinary free cortisol excretion in patients with depression and panic disorder. Biological Psychiatry, 25, 873-878.

Kelley, K. W., Dantzer, R., Mormede, P., Salmon, H., \& Aynaud, J. M. (1985). Conditioned taste aversion suppresses induction of delayed-type hypersensitivity immune reactions. Physiology \& Behavior, 34, 189-193. 
Khansari, D. N., Murgo, A. J., \& Faith, R. E. (1990). Effects of stress on the immune system. Immunology Today, 11, 170-175.

Kiecolt-Glaser, J. K., (1988). Methodological issues in behavioral immunology research with humans. Brain, Behavior, and Immunity, 2 , 67-78.

Kiecolt-Glaser, J. K., Cacioppo, J. T., Malarkey, W. B., \& Glaser, R. (1992). Acute psychological stressors and short-term immune changes: What, why, for whom, and to what extent? Psychosomatic Medicine, 54, 680-685.

Kiecolt-Glaser, J. K., Garner, W., Speicher, C., Penn, G. M., Holliday, J., \& Glaser, R. (1984). Psychosocial modifiers of immunocompetence in medical students. Psychosomatic Medicine, 46, 7-14.

Kiecolt-Glaser, J. K., \& Glaser, R. (1995). Psychoneuroimmunology and health consequences: data and shared mechanisms. Psychosomatic Medicine, 57, 269-274.

Kiecolt-Glaser, J. K., Glaser, R., Strain, E.C., Stout, J. C., Tarr, K. L., Holliday, J.E., \& Speicher, C.E. (1986). Modulation of cellular immunity in medical students. Journal of Behavioral Medicine, 9, 5-21.

Kiecolt-Glaser, J. K., Marucha, P. T., Malarkey, W. B., Mercado, A. M., \& Glaser, R. (1995). Slowing of wound healing by psychological stress. The Lancet, 346, 1194-1196.

Kiecolt-Glaser, J. K., Rickers, D., George, J., Messick, G., Speicher, C. E., Garner, W., \& Glaser, R. (1984). Urinary cortisol levels, cellular immunocompetency, and loneliness in psychiatric inpatients. Psychosomatic Medicine, 46, 15-23. 
Kim, M. J. (1987). Physiologic responses in health and illness: an overview. Annual Review of Nursing Research, 5, 79-104.

Kropiunigg, U. (1993). Basics in Psychoneuroimmunology. Annals of Medicine, 25, 473479.

Kuby, J. (1997). Immunology. ( $3^{\text {rd }}$ ed.). New York: W. H. Freeman and Company.

Kusnecov, A. W., Husband, A. J., \& King, M. G. (1990). The influence of dexamethasone on behaviorally conditioned immunomodulation and plasma corticosterone. Brain. Behavior, and Immunity, 4, 50-66.

Kvale, G., Hugdahl, K., Asbjornsen, A., Rosengren, B., Lote, K., \& Nordby, H. (1991). Anticipatory nausea and vomiting in cancer patients. Journal of Consulting and Clinical Psychology, 59, 894-898.

Labyak, S. E. \& Metzger, B. L. (1997). The effects of effleurage backrub on the physiological components of relaxation: a meta-analysis. Nursing Research, 46, 59-62.

Lambert, R. B., \& Lambert, N. K. (1995). The effects of humor on secretory Immunoglobulin A levels in school-aged children. Pediatric Nursing, 21, 16-19.

Landmann, R. M. A., Muller, F. B., Perini, C. H., Wesp, M., Erne, P., \& Buhler, F. B. (1984). Changes of immunoregulatory cells induced by psychological and physical stress: relationship to plasma catecholamine. Clinical Experiments in Immunology, 58, 127-135.

Laudenslager, M. L., Ryan, S. M., Drugan, R. C., Hyson, R. L., \& Maier, S. F. (1983). Coping and immunosuppression: inescapable but not escapable shock suppresses lymphocyte proliferation. Science, 221, 568-576. 
Lev, E. L. (1992). Patients' strategies for adapting to cancer treatment. Western Journal of Nursing Research, 14, 595-617.

Levy, R. H., Herberman, R., Lippman, M., \& d'Angelo, T. (1987). Correlation of stress factors with sustained depression of natural killer cell activity and predicted prognosis in patients with breast cancer. (1987). Journal of Clinical Oncology, 5, 348-353,

Linn, B. S., Linn, M. W., \& Jensen, J. (1981). Anxiety and immune responsiveness. Psychological Reports, 49, 969-970.

Longworthy, J. C. D. (1982). Psychophysiological effects of slow stroke back massage in normotensive females. Advances in Nursing Science, 4, 44-61.

Lundberg, U. (1976). Urban commuting: crowdedness and catecholamine excretion. Joumal of Human Stress, 2, 26-32.

Lysle, D. T., \& Cunnick, J. E. (1991). Stress-induced alterations of immune function: animal models. Clinical Immunology Newsletter, $11,177,180-183$.

Lysle, D. T., Cunnick, J. E., Fowler, H., \& Rabin, B. S. (1988). Pavlovian conditioning of shock-induced suppression of lymphocyte reactivity: acquisition, extinction, and preexposure effects. Life Sciences, 42, 2185-2194.

Lyles, J. N., Burish, T. G., Krozely, M. G., \& Oldham, R. K. (1982). Efficacy of relaxation training and guided imagery in reducing the aversiveness of cancer chemotherapy. Joumal of Consulting and Clinical Psychology, 50, 509-524.

Meek, S. S. (1993). Effects of slow stroke back massage on relaxation in hospice clients. IMAGE: Journal of Nursing Scholarship. 25, 17-21. 
Middelboe, T., Ovesen, L., Mortensen, E. L., \& Bech, P. (1994). Depressive symptoms in cancer patients undergoing chemotherapy: a psychometric analysis. Psychotherapy and Psychosomatics, 61, 171-177.

Miller, D. B., Thomas, J. R., Pacheco, N. D., \& Rollwagen, F. M. (1993). Natural killer cell cytotoxicity and T-cell proliferation is enhanced by avoidance behavior. Brain, Behavior, and Immunity, 7, 144-153.

Monsen, R. B., Floyd, R. L., \& Brookman, J. C. (1992). Stress-coping-adaptation: concepts for nursing. Nursing Forum, 27, 27-32.

Morrow, G. R. (1984). The assessment of nausea and vomiting. Past problems, current issues, and suggestions for futurr research. Cancer, 53, 2267-2277.

Morrow, G. R. (1992). Behavioral factors influencing the development and expression of chemotherapy induced side effects. British Journal of Cancer, 66(Suppl.. XIX) S54-S60.

Morrow, G. R., Asbury, R., Hammon, S., Dobkin, P., Caruso, L., Pandya, K., \& Rosenthal, S. (1992). Comparing the effectiveness of behavioral treatment for chemotherapyinduced nausea and vomiting when administered by oncologists, oncology nurses, and clinical psychologists. Health Psychology, 11, 250-256.

Morrow, G. R., \& Morrell, C. (1982). Behavioral treatment for nausea and vomiting induced by cancer chemotherapy. The New England Journal of Medicine, 307, 1476-1480.

Munroe, B. M., \& Page, E. B. (1993). Statistical methods for health care research. $\left(2^{\text {nd }}\right.$ ed.). Philadelphia: J. b. Lippincott Company. 
Mynchenberg, T. L., \& Dungan, J. M. (1995). A relaxation protocol to reduce patient anxiety. Dimensions of Critical Care Nursing, 14, 78-85.

Nerenz, D. R., Levendthal, H., Easterling, D. V., \& Love, R. R. (1986). Anxiety and drug taste as predictors of anticipatory nausea in cancer chemotherapy. Journal of Clinical Oncology, 4 , 224-233.

Newell, S., Swanson-Fisher, R. W., Girgis, A., \& Ackland, A. (1999). The physical and psycho-social experiences of patients attending an outpatient medical oncology department: a cross-sectional study. European Journal of Cancer Care, 8, 73-82.

Nguyen, T. V. (1991). Mind, brain, and immunity: a critical review. Holistic Nursing Practice, 5, 1-9.

Nicholas, D. R., \& Hollandsworth, J. G. (1986). Assessment of anticipatory nausea and vomiting in cancer patients undergoing chemotherapy: theoretical and methodological considerations. Joumal of Psychosocial Oncology, 4, 61-75.

Nicholas, P. K., \& Webster, A. (1996). A behavioral medicine intervention in persons with HIV. Clinical Nursing Research, 5, 391-406.

Okun, A., Stein, R. E., Bauman, L. J., \& Silver, E. J. (1996). Content validity of the Psychiatric Symptom Index, CES-Depression Scale, and State-Trait anxiety Inventory from the perspective of DSM-IV. Psychological Reports, 79, 1059-1069.

O'Leary, A., \& Miller, S. M. (1991). Stress, immune function, and health: early settlement of a new frontier. Clinical Immunology Newsletter, 11, 177-180.

Otto, S. E. (1997). Oncology nursing ( $3^{\text {rd }}$ ed.). St. Louis: Mosby 
Pickett, M. (1991). Determinants of anticipatory nausea and anticipatory vomiting in adults receiving cancer chemotherapy. Cancer Nursing, 14, 334-343.

Polit, D. F., \& Hungler, B. P. (1991). Nursing research. Principles and methods. (4 ${ }^{\text {th }}$ ed.). Philadelphia: J. B. Lippincott Company.

Rabin, B. S. (1999). Stress, immune function and health. The connection. New York: A John Wiley \& Sons, Inc., Publication.

Rawlins, R. P., Williams, S. R. \& Beck, C. K. (1993). Mental health - psychiatric nursing. A holistic life-cycle approach. St. Louis: Mosby.

Razavi, D., Delvaux, N., Farvacques, C., De Brier, F., Van Heer, C., Kaufman, L., Derde, P., Beauduin, M., \& Piccart, M. (1993). Prevention of adjustment disorders and anticipatory nausea secondary to adjuvant chemotherapy: a double-blind, placebo-controlled study assessing the usefulness of Alprazolam. Journal of Clinical Oncology, 11, 1384-1390.

Redd, W. H., Andersen, G. V., \& Minagawa, R. Y . (1982). Hypnotic control of anticipatory emesis in patients receiving cancer chemotherapy. Journal of Consulting and Clinical Psychology, 50, 14-19.

Redd, W. H., \& Andrykowski, M. A. (1982). Behavioral intervention in cancer treatment: controlling aversion reactions to chemotherapy. Journal of Consulting and Clinical Psychology, 50, 1018-1029.

Rhiner, M., Dean, G. E., \& Ducharme, S. (1996). Home Health Care Management \& Practice, $8,41-47$. 
Rhodes, V. A., Watson, P. M., \& Johnson, M. H. (1986). Association of chemotherapy related nausea and vomiting with pretreatment and posttreatment anxiety. Oncology Nursing Forum, 13, 41-47.

Rice, P. L. (1992). Stress and Health. $2^{\text {nd }}$ Ed., Pacific Grove: Brooks/Cole Publishing Company.

Richarsdon, J. L., Landring, H., \& Marks, G. (1994). Does psychological status influence cancer patient survival? A case still in need of evidence. In C. E. Lewis, C. O'Sullivan, \& J. Barraclough, (Eds.), The psychoimmunology of cancer. Mind and body in the fight for survival? (pp. 228-245). Oxford: Oxford University Press.

Robinson, L. (1990). Stress and anxiety. Nursing Clinics of North America, 25, 935-943.

Roudebush, R. E. \& Bryant, H. U. (1991). Conditioned immunosuppression of a murine delayed type hypersensitivity response: Dissociation from corticosterone elevation. Brain. Behavior, and Immunity, 5, 308-317.

Sabo, C. E., \& Michael, S. R. (1996). The influence of personal message with music on anxiety and side effects associated with chemotherapy. Cancer Nursing, 19, 283-289.

Sabbioni, M. E. E. (1993). Psychoneuroimmunological issues in psycho-oncology. Cancer Investigation, $11,440-450$.

Schedlowski, M., Jacobs, R., Stratmann, G., Richter, S., Hadicke, A., Twews, U., Wagner, T., \& Schmidt, R. (1993). Changes of natural killer cells during acute psychological stress. Journal of Clinical Immunology, 13, 119-126. 
Schedlowski, M., Twews, U., \& H.-J. Schmoll. (1994). The effects of psychological intervention on cortisol levels and leukocyte numbers in the peripheral blood of breast cancer patients. In C. E. Lewis, C. O'Sullivan, \& J. Barraclough, (Eds.), The psychoimmunology of cancer. Mind and body in the fight for survival? (pp. 336-348). Oxford: Oxford University Press.

Schwab, K. O., Heubel, G., \& Bartels, H. (1992). Free epinephrine, norepinephrine and dopamine in saliva and plasma of healthy adults. European Journal of Clinical Chemistry and Clinical Biochemistry, 30, 541-544.

Scott, D. W., Donahue, D. C., Mastrovito, R. C., \& Hakes, T. B. (1986). Comparative trial of clinical relaxation and an antiemetic drug regimen in reducing chemotherapy-related nausea and vomiting. Cancer Nursing, 9, 178-187.

Seligman, L. (1996). Promoting a fighting spirit. Psychotherapy for cancer patients, survivors, and their families. San Francisco: Jossey-Bass Publishers.

Siconolfi, L. A. (1994). The forgotten system: endocrine dysfunction during multiple organ dysfunction. Critical Care Nursing Quarterly, 16, 16-26.

Sims, S. (1987). Relaxation training as a technique for helping patients cope with the experience of cancer: a selective review of the literature. Joumal of Advanced Nursing, 12, 583-591.

Snyder, B. K., Roghmann, K. J., \& Sigal, L. H. (1993). Stress and psychosocial factors: effects on primary cellular immune response. Journal of Behavioral Medicine, 16, 143-161. Sparacino, J. (1982). Blood pressure, stress, and mental health. Nursing Research, 31, 89-94. Speilberger, C. D. (1983). Test manual for state-trait anxiety inventory. Palo Alto, CA: Consulting Psychological Press. 
Stuart, G. W., \& Sundeen, S. J. (1995). Principles and practice of psychiatric nursing/ with quick psychopharmacology reference. St. Louis: Mosby.

Taylor, D. N. (1995). Effects of a behavioral stress-management program on anxiety, mood, self-esteem, and T-cell count in HIV-positive men. Psychological Reports, 76, 451-457.

Tyler, D. O., Winslow, E. H., Clark, A. P. \& White, K. M. (1990). Effects of a 1-minute back rub on mixed venous oxygen saturation and heart rate in critically ill patients. Heart \& Lung, $19,562-569$.

Vasterling, J., Jenkins, R. A., Tope, D. M., \& Burish, T. G. (1993). Cognitive distraction and relaxation training for the control of side effects due to cancer chemotherapy. Journal of Behavioral Medicine, 16, 65-80.

Vedhara, K., Fox, J. D., \& Wang, E. C. Y. (1999). The measurement of stress-related immune dysfunction in Psychoneuroimmunology. Neuroscience and Biobehavioral Reviews, 23, $699-715$

Walker, L. G., Johnson, V. C., Whiting, P. H., Heys, S. D., \& Ermin, O. (1993). Immune response to stress. British Journal of Cancer, 67(Supp. 20). 140.

Walker, L. G., Walker, M. B., Ogston, K., Heys, S. D., Ah-See, A. K., Miller, I. D., Hutcheon, A. W., Sarkar, T. K., \& Eremin, O. (1999). Psychological, clinical and pathological effects of relaxation training and guided imagery during primary chemotherapy. British Journal of Cancer, 80, 262-268.

Watson, M., McCarron, J., \& Law, M. (1992). Anticipatory nausea and emesis, and psychological morbidity: assessment of prevalence among out-patients on mild to moderate chemotherapy regimens. British Journal of Cancer, 55, 682-866. 
Weizman, R. \& Bessler, H. (1999). Cytokines: stress and immunity - an overview. In N. P. Plotnikoff, R. E. Faith, A. J. Murgo, \& R. A. Good (Eds.), Cytokines stress and immunity (pp 115). Boca Raton: CRC Press.

Westerman, J., \& Pabst, R. (1992). Distribution of lymphocyte subsets and natural killer cells in the human body. Clinical Investigation. 70, 539-544.

Wilson, H. S., \& Kneisle, C. R. (1992). Psychiatric nursing. Menlo Park, California: Addison-Wesley.

Zachariae, R, Kristensen, J. S., Hokland, P., Ellagaard, J., Metze, E., \& Hokland, M. (1990). Effect of psychological intervention in the form of relaxation and guided imagery on cellular immune function in normal healthy subjects. Psychotherapy and Psychosomatics, 54, 32-39.

Zeller, J. M., McCaine, N. L., \& Swanson, B. (1996). Psychoneuroimmunology: an emerging framework for nursing research. Journal of Advanced Nursing, 23, 657-664.

Zorzet, S., Perissin, L., Rapozzi, V., \& Giraldi, T. (1998). Restraint stress reduces the antitumor efficacy of Cyclophosphamide in tumor-bearing mice. Brain. Behavior, and Immunity $12,23-33$ 
Appendix A

\section{SCRIPT, PROGRESSIVE MUSCLE RELAXATION AUDIOTAPE}

The purpose of this progressive muscle relaxation exercise is to help you relax as you undergo chemotherapy. Sit or lie down in a comfortable position in a quiet place. Make sure that your body feels comfortable. Let any stresses and anxieties go for the next few minutes. As your muscles relax, let your mind relax as well. As you practice relaxing, breathe in and out slowly, deeply, and easily. Concentrate on how your muscles feel when they are relaxed.

Let your eyes close and take a deep breath in, counting to five

$$
\begin{aligned}
& 1,2,3,4,5 \\
& \text { now breath out slowly }
\end{aligned}
$$

Breath in and out slowly four more times

In

and out

In

out

In

and out

In

out

Slowly clench your left hand

tighter - and hold it(12345) - think of what the tension feels like

and relax - feel the difference

Clench your right fist - hold it -1,2,3,4,5 -

Relax your fist, notice the difference

Extend your fingers of both hands as far as you can - $1,2,3,4,5$

and relax

Stretch your fingers again - $1,2,3,4,5$

and relax them, feel the difference

notice how relaxed your hands feel

Now tighten the muscles in your arms

hold them tense - $1,2,3,4,5$

and let your arms relax

Tighten the muscles in your arms again

hold them tense

and let your arms relax

feel the difference and enjoy the contrast 
Shrug your shoulder muscles and make them tense

tense them

and let them relax completely

Pull your shoulders up and back - 1,2,3,4,5

now relax

Push your shoulders forward, keep them tense $1,2,3,4,5$

and relax

feel the difference when you relax

Shrug your shoulders again and tense them

hold it ....- and relax

concentrate on how relaxed your shoulder muscles feel

Now tense the muscles of your forehead

frown and feel the tension as you frown, tense - $1,2,3,4,5$

and relax your forehead

Frown again, keep it tense

and relax

feel the difference when you are relaxed

Raise your eyebrows and hold it - 1,2,3,4,5

now relax

Do it again, raise them

and relax

Tense the muscles around your eyes

squeeze your eyes shut - $1,2,3,4,5$

and let them relax

Squeeze your eyes shut again

hold it

and relax

Concentrate on how those muscles feel when they are relaxed

Pinch your lips together and tense the muscles of your cheeks and lips

hold them tense $-1,2,3,4,5$

and relax, feel the difference

Open your mouth as wide as you can - 1,2,3,4,5

and let your mouth relax

Bring your tongue up to the top of your mouth

keep it tense, hold it $-1,2,3,4,5$

let it relax

Now tighten your jaw muscles

hold it tight, tight $-1,2,3,4,5$

and relax

enjoy the feeling of your jaw being relaxed

Concentrate on how relaxed your face feels 
Now take in a deep slow breath, breath in as deep as you can

feel how your chest muscles stretch

hold it $-1,2,3,4,5$

and breathe out as much you can,

relax

Breathe in deeply again, deeper

and out

relax

Tighten the muscles in your back as much as you can

tighten your muscles $-1,2,3,4,5$

and relax

feel the difference as you relax

tighten your back muscles again

hold it

and relax

enjoy the feeling of being relaxed

Now tighten the muscles of your stomach

push your stomach out as much as you can - 1,2,3,4,5

and relax

tighten your stomach muscles again

push it out

and relax

Pull your stomach in as far as you can

hold it $-1,2,3,4,5$

and relax

pull in your stomach muscles again

and relax

Notice how relaxed your stomach feels

Now tighten the muscles of your buttocks

tighten them - $1,2,3,4,5$

feel the tension

and relax

tighten your buttocks muscles again

tighter and hold it

now relax

Tighten the muscles of your left thigh

and lift your legs up slightly

hold your let up and feel your nuscles tight $-1,2,3,4,5$

and relax as you breathe slowly and easily 
Now tense your right thigh

lift your right leg up slightly

and hold it

feel how tense your muscles feel

and relax

feel the difference

Now push your left leg down as hard as you can

feel the muscles in your legs tighten - $1,2,3,4,5$

hold it

and relax

Push your right leg down

tense your muscles

and relax

notice how your legs feel relaxed

Bend both your ankles and point your toes as far as you can

hold it and feel the tension in your calf muscles - 1,2,3,4,5

relax your muscles

do it again, and point your toes

relax

and feel your calf muscles relaxed

Now pull your toes up as far as you can

hold it $-1,2,3,4,5$

and relax. Feel the difference in your muscles as you relax

Tense your toes again - Stretch them up as far as you can

press your toes against the top of your shoes $-1,2,3,4,5$

now relax them

Press your toes down against your shoes them down as far as you can

press and hold $-1,2,3,4,5$

and relax

again press your toes down

and relax them

feel how relaxed your toes feel

Now breathe easily and deeply several times

in

and out

and concentrate on how relaxed your muscles feel

feel your toes relaxed

feel your ankles relaxed 
feel your calves relaxed

feel your thighs relaxed

feel your buttocks relaxed

feel your stomach relaxed

feel your shoulders relaxed

feel your arms relaxed

feel your hands relaxed

feel your fingers relaxed

feel your neck relaxed

feel your face relaxed

Breathe in deeply and feel your breathing muscles expand

breathe out and feel them relax

Do it again and notice the feeling of your muscles relaxed

Let yourself stay as you are for a few moments and enjoy the feeling of being relaxed. Breathe easily, and deeply. Think of how you will relax your muscles when you receive your chemotherapy.

When you are ready move your focus back to your day and what you will be doing next. Get up when you feel like it. 
Appendix C

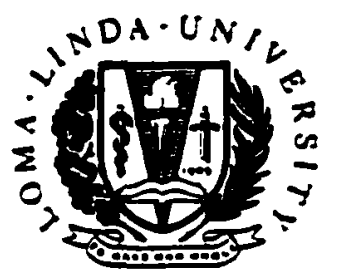

LOMA LivDA UnVERSITY

Offies of Sponsoned Rescaret

January 8. 1996
Lome Liada, Calijomie 92350

(909) $821-1531$

F.LX: $(909)$-78-413!

Patricin K. Taylor Pothier, RN, MS

School of Nursing

Lomn Linda University

Dear Ms. Pothier.

IRB Project Dir9600I

The application for a study encitled "The Effect of a Nursing Relaxation Intervention on Neuro-Immune Responses of Persons Undergoing Chemotherapy" was reviewed administratively on behalf of the Lnstitutional Review Board of Loma Linda University on January 8, 1996.

The actions of the review are as follows:

- The subjeces are at minimum risk

- The protocol is approved from January 8, 1996 wo January 7. 1997.

- Approval is based on condition that exelusion criteria in the protocol assure that no individual will have more than a tocal of $450 \mathrm{ml}$ of blood drawn for any purpose within an eight-week period.

The enclosed consent form bas been validated with the IRB approval stnmp. This now becomes your oricinl conseat form for the dates specified and should be used as a master copy for enrolling subjects.

If there are anodificarions to the proposed resenreh protocol or problems arising from the study, plense notify the Board in writing. If you bave any questions. please feel free to conenct us.

You are required to provide a progress reporr on this study in one year indicating the number of subjects enrolled.

Best wishes for success in this project.

Sincerely yours.

G. Hilyón Sankel. M.D.

Chinimin

Institutional Review Boand

Encl. 


\section{Appendix D}

\section{INFORMED CONSENT FOR PARTICIPATION IN A CLINICAL RESEARCH STUDY ON THE EFFECT OF A NURSING RELAXATION INTERVENTION ON NERUO-IMMUNE RESPONSES OF PERSONS UNDERGOING CHEMOTHERAPY}

I have been invited to participate in a research study. Before I give my consent, I will read the following and ask whatever questions I may have.

\section{PURPOSE OF THE STUDY}

The purpose of this study is to examine the effects of relaxation, including slow-stroke back massage and progressive muscle relaxation, on the immune system of persons who are undergoing chemotherapy. The immune system is made up of cells which function to protect the body against problems like infection. Certain drugs used for chemotherapy may affect cells involved in the immune system. The immune system may be sensitive to stressful situations. Some people might feel chemotherapy is stressful because of the side effects that may accompany it. This study will try to find out whether a change in immune function occurs along with chemotherapy. It will also study what effect relaxation has on some people who are having chemotherapy.

This will be done by examining the immune status before starting chemotherapy and again after three doses of chemotherapy. Half of the people will be in a part of the study that includes back massage and progressive muscle relaxation along with the usual chemotherapy program. The other half will be in a part of the study that involves the usual chemotherapy program alone. After three treatments with chemotherapy, the immune function will be studied to see if there is any difference between people who had the relaxation with chemotherapy and those who had the usual chemotherapy program.

I have been told that nothing in this study will in any way affect or modify the medication and treatment prescribed by my physician.

\section{PROCEDURE}

I understand that if I participate in this study I will be randomly assigned, by my drawing a slip of paper out of a box, to a Group A that gets standard care and treatment or to a Group B that gets standard care and treatment plus relaxation training.

I understand that I will be asked to have an assessment done before starting chemotherapy. This will involve filling out a form asking questions about feelings of anxiety, and having about two tablespoons more of blood drawn with my regular blood tests. My blood pressure and heart rate will also be measured. Information about my general health status, medications I need to take, and the kind of cancer will be taken from my medical record. At the first three chemotherapy treatments I will be asked to answer questions about my diet.

If I am in Group A, I will have my chemotherapy according to a usual schedule. If I am in Group B, I will also have my chemotherapy according to usual schedule. In addition, I will also have a slow-stroke back massage just before four chemotherapy treatments. I will also be given an audio tape of instructions on progressive muscle relaxation and asked to practice the procedure for $\mathbf{3 0}$ minutes at least once a day 
(page 2 of 3 )

INFORMED CONSENT FOR PARTICIPATION IN A CLINICAL RESEARCH STUDY ON THE EFFECT OF A NURSING RELAXATIONINTERVENTIONON NEURO-IMMUNE RESPONSES OF PERSONS UNDERGOING CHEMOTHERAPY

After the second chemotherapy treatment, and just before the third treatment, I will have about two tablespoons of blood drawn with my regular blood tests. My blood pressure and heart rate will be measured. I will fill out a form asking about feelings of anxiety. I will also be asked to fill out a form that asks questions about nausea and vomiting associated with chemotherapy treatment.

Just before the fourth chemotherapy treatment, I will have about two tablespoons of blood drawn along with my regular blood tests. I will fill out a form asking questions about anxiety. While I am having the chemotherapy treatment, I will also fill out a final form asking questions about nausea and vomiting. After that, I will be finished with participation in the study.

\section{TIME}

Because this research involves my participation during 4 courses of chemotherapy, I understand that it will take about 12 weeks from start to finish. Filling out the form asking questions about anxious feelings will take about 20 minutes. Filling out the form asking questions about nausea and vomiting will take about 10 minutes. The dietary questionnaire will take about 10 minutes. If I am in the group that uses the relaxation, I will get a 10-minute back massage before my chemotherapy treatment. I will also be asked to spend 30 minutes a day listening to a tape.

\section{RISKS}

I have been told that no aspect of this study will change in any way the treatment ordered by my physician. The Institutional Review Board, a committee that carefully reviews all research done at Loma Linda University, has determined that participating in this study exposes me to minimal risk for participating in the study. I understand that the possible risk to me is minor pain and bruising at the site of the needle insertion for drawing a blood sample.

\section{BENEFITS OF THE RESEARCH}

I have been told that I may not directly benefit from this study. However, it is hoped that the results of the research will help in planning cares that may help persons undergoing chemotherapy in the future. Results of blood studies will be sent to my physician.

\section{PARTICIPANTS RIGHTS}

My participation in this study is completely voluntary. I understand that I may withdraw at any time without any penalty. I have been told that my decision of whether to participate or not, or a decision to withdraw from the study will not affect my treatment now or in the future. I understand that there is no agreement, either written or verbal, beyond that expressed in this consent form.

\section{CONFIDENTIALITY}

I understand that my confidentiality will be protected and that information that is identifiable will be kept in a locked file cabinet in the researcher's office. I have been told that any published document that results from this study will not reveal my identity. I have been told that the Department of Health and Human Services or their designated representative may inspect the records relating to my participation in this study.

\section{COST}

There will be no additional cost to me as a result of participation in this study. There will be no reimbursement to me for participating in this study. 
(page 3 of 3)

INFORMED CONSENT FOR PARTICIPATION IN A CLINICAL RESEARCH STUDY ON THE EFFECT OF A NURSING RELAXATION INTERVENTION ON NEURO-IMMUNE RESPONSES OF PERSONS UNDERGOING CHEMOTHERAPY

\section{CONTACT PERSON}

If at any time I wish to contact an impartial third party not associated with this study or with my medical care, I may contact Jean Frankhanel, Patient Representative, at Loma Linda University Medical Center, Loma Linda, California 92350, telephone (909) 824-4648, for assistance.

\section{DNFORMED CONSENT STATEMENT}

"I have read the contents of the consent form and have listened to the verbal explanation given by the investigator, Patricia Pothier, RN, MS, a doctoral candidate at the Philip Y. Hahn School of Nursing, University of San Diego. My questions concerning this study have been answered to my satisfaction. I hereby give my voluntary consent to participate in this study. Signing this consent form does not waive my rights, nor does it release the investigators, institution, or sponsors from their responsibilities.

I may call Patricia Pothier, RN, during routine office hours at (909) 824-4360, Ext. 45480, or leave a message at that number on an answering machine. I can also page her by calling (909) 824-0800. Pager Number 5023.

I have been given a copy of this consent form. I have received a copy of the California Experimental Subject's Bill of Rights and have had these rights explained to me."

Signature

$$
\text { Date }
$$

\section{Witness}

"I have reviewed the contents of the consent form and the California Experimental Subject's Bill of Rights with the person signing above. I have explained the potential risks and benefits of the study."

Signature of Investigator

Date

_ Indicate by checking here if you would like results of the study. (If yes, please print your name and mailing address below.)

Name:

Address: 
Appendix E

\section{CALIFORNIA EXPERIMENTAL SUBJECT'S BILL OF RIGHTS}

You have been asked to participate as a subject in an experimental clinical procedure. Before you decide whether you want to participate in the experimental procedure, you have a right to:

1. Be informed of the nature and purpose of the experiment;

2. Be given an explanation of the procedures to be followed in the medical experiment, and any drug or device to be utilized;

3. Be given a description of any attendant discomforts and risks reasonably to be expected from your participation in the experiment;

4. Be given an explanation of any benefits reasonably to be expected from your participation in the experiment;

5. Be given a disclosure of any appropriate alternative procedures, drugs, or devices that might be advantageous to you and their relative risks and benefits;

6. Be informed of the avenues of medical treatment, if any, available to you after the experimental procedure if complications should arise;

7. Be given an opportunity to ask any questions concerning the medical experiment or the procedures involved;

8. Be instructed that consent to participate in the experimental procedure may be withdrawn at any time and that you may discontinue participation in the medical experiment without prejudice;

9. Be given a copy of this form and the signed and dated written consent form; and

10. Be given the opportunity to decide to consent or not to consent to the medical experiment without the intervention of any element of force, fraud, deceit, duress, coercion, or undue influence on your decision.

I have carefully read the information contained above in the "California Experimental Subject's Bill of Rights" and I understand fully my rights as a potential subject in a medical experiment involving people as subjects.

\section{Date}

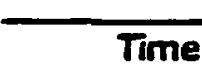

Patient

Parent/Legal Guardian

If signed by other than the patient, indicate relationship: 


\section{Appendix F}

\section{DATA SHEET}

\section{Identification}

1. Research ID \#

2. FMO ID \#

Last Name

First Name

3. Age

4. Research Status (Control = 1, Experimental = 2)

Health History $($ Yes $=1, \mathrm{NO}=2)$

5. HTN

6. Diabetes

7. Other major health problem (specify)

Medication currently taking ( $Y e s=1, \mathrm{NO}=2$ )

8. _ Medication for HTN (specify

9. Hypoglycemic agent

10. _ Cardiac medication (specify

11. Did client require GSF during chemo? (YES $=1, \mathrm{NO}=2$ )

\section{Cancer Diagnosis}

site

extent/stage

cellular composition

Surgical Intervention before chemotherapy (YES $=1, \mathrm{NO}=2$ )

12. Lumpectomy

13. _ Simple mastectomy

14. Modified radical mastectomy

15. Chemotherapy before surgery

\section{Nutritional analysis}

16. Weight in lbs. before chemotherapy

17. Weight in lbs. before dose 3

18. Weight in lbs. before dose 4

19. Serum albumin before chemotherapy

20. Serum albumin before dose 3

21. Serum albumin before dose 4 
Days between chemotherapy doses

22. \# days between dose 1 and dose 3

23. \# days between dose 1 and dose 4

\section{STAI}

24. Baseline State

25. Baseline Trait

26. State Score prior to dose 3

27. Trait Score prior to dose 3

28. State Score prior to dose 4

29. Trait Score prior to dose 4

MANE (YES $=1$, NO = 2)

30. Nausea prior to dose 3

31. Nausea/vomiting post dose 3

32. Nausea prior to dose 4

Catecholamines at baseline

33. Norepinephrine (PG/MI)

34. Epinephrine (PG/ML)

35. Dopamine (PG/ML)

Catecholamines prior to 3 rd dose

36. Norepinephrine (PG/ML)

37. Epinephrine (PG/ML)

38. Dopamine (PG/ML)

Catecholamines prior to 4 th dose

39. Norepinephrine (PG/ML)

40. Epinephrine (PG/ML)

41. Dopamine (PG/ML)

Total Complement draws 1, 2, \& 3

42. at baseline

43. prior to 3rd dose

44. prior to 4th dose

Serum Immunoglobulins: baseline

45. IGG (MG/DL)

46. IGA (MG/DL)

47. IGM (MG/DL)

48. Kappa

49. Lambda

50. $\mathrm{K} /$ Ratio 
Serum Immunoglobulins: prior to 3rd dose

51. IGG (MG/DL)

52. IGA (MG/DL)

53. IGM (MG/DL)

54. Kappa

55. Lambda

56. $\mathrm{K} / \mathrm{L}$ Ratio

Serum Immunoglobulins: prior to 4 th dose

57. IGG (MG/DL)

58. IGA (MG/DL)

59. IGM (MG/DL)

60. Kappa

61. Lambda

62. K/ Ratio

Flow Cytometry Phenotyping: baseline

CD45-F/CD14-PE

64. Lymphocyte Purity

CD3-F/CD20-PE

65. T- Lymphocytes CD3+

66. B- Lymphocytes CD20+

CD3-F/CD25-PE

67. T-Lymphocytes $\mathrm{CD} 3+$

68. IL-2 Activated T Lymphocytes $\mathrm{CD} 3+25+$

CD3-F/CD16,56-PE

69. T-Lymphocytes CD3+

70. NK Cells CD3-CD16+56+

71. CD3 Average

Flow Cytometry Phenotyping: prior to 3rd dose

CD45-F/CD14-PE

72. Lymphocyte purity

CD3-F/CD20-PE

73. T Lymphocytes CD3+

74. B Lymphocytes CD20+

CD3-F/CD25-PE

75. T-Lymphocytes $\mathrm{CD} 3+$

76. IL-2 Activated T Lymphocytes CD3+25+

CD3-F/CD16,56-PE

77. T-Lymphocytes CD3+

78. NK Cells CD3-CD16+56+

79. CD3 Average 
Flow Cytometry Phenotyping: prior to 4 th dose

CD45-F/CD14-PE

80. Lymphocyte purity

CD3-F/CD20-PE

81. T Lymphocytes CD3+

82. B Lymphocytes CD20+

CD3-F/CD25-PE

83. T-Lymphocytes CD3+

84. IL-2 Activated T Lymphocytes CD3+25+

CD3-F/CD16,56-PE

85. T-Lymphocytes CD3+

86. NK Cells CD3-CD16+56+

87. CD3 Average

Mitogen Stimulation Assay (whole blood): Baseline

88. PHA $12.5 \mathrm{ug} / \mathrm{mL}$

89. PHA $3.125 \mathrm{ug} / \mathrm{mL}$

90. PHA $1.56 \mathrm{ug} / \mathrm{mL}$

Mitogen Stimulation Assay (whole blood): prior to 3rd dose

91. PHA $12.5 \mathrm{ug} / \mathrm{mL}$

92. PHA $3.125 \mathrm{ug} / \mathrm{mL}$

93. PHA $1.56 \mathrm{ug} / \mathrm{mL}$

Mitogen Stimulation Assay (whole blood): prior to 4 th dose

94. PHA $12.5 \mathrm{ug} / \mathrm{mL}$

95. PHA $3.125 \mathrm{ug} / \mathrm{mL}$

96. PHA $1.56 \mathrm{ug} / \mathrm{mL}$

NK Cells

97. NK baseline

98. NK prior to 3rd dose

99. NK prior to 4th dose

(Leave space here for additional NK data

Massage ( 0 = none, 1 = not relaxing, $2=$ somewhat relaxing, $3=$ quite relaxing, 4=very relaxing) 110.

Progressive Muscle Relaxation ( $0=$ none, $1=$ occasionally, $2=$ about $1 / 2$ the days, $3=$ usually, 4 = almost every day)

111 .

COMMENTS: (What did client say about response to therapy? How did client respond to appointments? Any verbalized stressors?) 
Appendix G

Descriptive Statistics for Immune Function Tests for Subjects Not Requiring GCSF

\begin{tabular}{|c|c|c|c|c|c|c|c|c|c|}
\hline \multirow{2}{*}{$\begin{array}{l}\text { Test \& \# } \\
\text { Mean } \\
\text { (Ref. Range) }\end{array}$} & \multicolumn{3}{|c|}{ Mean for } & Stand. & \multicolumn{2}{|c|}{ Mean for } & \multicolumn{2}{|c|}{ Stand. } & \multirow[b]{2}{*}{ p } \\
\hline & \multicolumn{2}{|c|}{ Control } & Dev. & \multicolumn{2}{|c|}{ Experimental } & Dev. & Diff. & t-value & \\
\hline \multicolumn{10}{|c|}{ IgG (69+-1618 mg/dl) } \\
\hline Baseline \#1 & $(n=2)$ & 1053.000 & 176.60 & $5(n=3)$ & 1343.333 & 326.2412 & -290.333 & -1.113 & .347 \\
\hline Measure \#2 & $(n=1)$ & 984.000 & & $(n=3)$ & 1046.666 & 47.25 & -62.666 & -1.144 & .370 \\
\hline Measure \#3 & $(n=2)$ & 1090.000 & 0.00 & $0(n=4)$ & 1129.250 & 330.487 & -39.250 & -0.154 & .882 \\
\hline \multicolumn{10}{|c|}{$\operatorname{IgA}(68-378 \mathrm{mg} / \mathrm{dl})$} \\
\hline Baseline \#I & $(n=2)$ & 188.500 & 47.37 & $(n=3)$ & 354.333 & 235.602 & -156.833 & -0.884 & .442 \\
\hline Measure \#2 & $(n=0)$ & \multicolumn{8}{|c|}{ not able to calculate because of missing data } \\
\hline Measure \#3 & $(n=2)$ & 195.000 & 50.91 & $1(n=4)$ & 313.7500 & 218.374 & -118.750 & -0.719 & .512 \\
\hline \multicolumn{10}{|c|}{$\operatorname{lgM}(60-263 \mathrm{mg} / \mathrm{dl})$} \\
\hline Baseline \#1 & $(n=2$ & 102.250 & 32.17 & $(n=3)$ & 232.000 & 73.082 & -129.750 & -2.740 & .107 \\
\hline Measure \#2 & $(n=0)$ & \multicolumn{8}{|c|}{ not able to calculate due to missing data } \\
\hline Measure \#3 & $(n=2)$ & 89.450 & 40.37 & $5(n=4)$ & 179.500 & 40.763 & -90.050 & -2.557 & .063 \\
\hline \multicolumn{10}{|c|}{ T Lymphocyte CD3+ \% (61-87\%) } \\
\hline Baseline \#1 & $(n=2)$ & 82.500 & 3.53 & $5(n=5)$ & 73.200 & 5.069 & 9.300 & 2.315 & .069 \\
\hline Measure \#2 & $(n=1)$ & 93.00 & & $(n=3)$ & 77.333 & 14.153 & 15.666 & 0.959 & .439 \\
\hline Measure \#3 & $(n=2)$ & 95.000 & 2.828 & $3(n=4)$ & 81.500 & 8.062 & 13.500 & 2.188 & .094 \\
\hline \multicolumn{10}{|c|}{ T Lymphocyte CD3+\# (1.642-2.3+2 b/L) } \\
\hline Baseline \#1 & $(n=2)$ & 1.515 & 0.13 & $(n=5)$ & 1.776 & 2.067 & -0.261 & -0.169 & .873 \\
\hline Measure \#2 & $(n=1)$ & 1.030 & & $(n=3)$ & 1.006 & 0.456 & 2.333 & 0.044 & .969 \\
\hline Measure \#3 & $(n=2)$ & 1.155 & 0.34 & $5(n=4)$ & 1.027 & 0.341 & 0.127 & 0.430 & .690 \\
\hline \multicolumn{10}{|c|}{ B Lymphocyte CD20+ \% (2-19\%) } \\
\hline Baseline \#1 & $(n=2)$ & 14.500 & $0.70^{\circ}$ & $(n=5)$ & 14.000 & 4.301 & 0.500 & 0.155 & .883 \\
\hline Measure \#2 & $(n=1)$ & 0.000 & 0 & $(n=3)$ & 10.666 & 16.743 & -10.666 & -0.552 & .637 \\
\hline Measure \#3 & $(n=2)$ & 0.000 & 0 & $(n=4)$ & 0.750 & 0.957 & -0.750 & -1.044 & .355 \\
\hline \multicolumn{10}{|c|}{ B Lymphocyte CD20+ \# (0.027-0.458 b/L) } \\
\hline Baseline \#1 & $(n=2)$ & 0.270 & 5.65 & $(n=5)$ & 0.342 & 0.440 & -7.200 & -0.218 & .836 \\
\hline Measure \#2 & $(n=1)$ & 0.000 & 0 & $(n=3)$ & 0.200 & 0.329 & -0.200 & -0.526 & .651 \\
\hline Measure \#3 & $(n=2)$ & 0.000 & 0.000 & $(n=4)$ & 1.250 & 1.893 & -1.250 & -0.880 & .428 \\
\hline \multicolumn{10}{|c|}{ I-2 Activated Lymph's \% (15-39\%) } \\
\hline Baseline \#1 & $(n=2)$ & 31.000 & 8.38 & $(n=5)$ & 17.200 & 5.357 & 13.800 & 1.733 & .477 \\
\hline Measure \#2 & $(n=1)$ & 53.00 & & $(n=3)$ & 16.666 & 2.516 & 36.333 & 12.503 & $.006^{*}$ \\
\hline Measure \#3 & $(n=2)$ & 28.500 & 14.849 & $(n=4)$ & 22.000 & 5.887 & 6.500 & 0.833 & .452 \\
\hline \multicolumn{10}{|c|}{ IL-2 Activated Lymph's \# (0.072-2.287 b/L) } \\
\hline Baseline \# I & $(n=2)$ & 0.555 & 0.267 & $(n=5)$ & 0.472 & 0.646 & 7.800 & 0.158 & .881 \\
\hline Measure \#2 & $(n=1)$ & 0.580 & & $(n=3)$ & 0.203 & 0.101 & 0.376 & 3.225 & .084 \\
\hline Measure \#3 & $\begin{array}{l}(n=2) \\
05)\end{array}$ & 0.315 & 7.778 & $(n=4)$ & 0.275 & 8.888 & 4.000 & 0.536 & .621 \\
\hline
\end{tabular}




\section{(Continued)}

\begin{tabular}{|c|c|c|c|c|c|c|c|c|}
\hline $\begin{array}{l}\text { Test \& \# } \\
\text { (Ref. Range) }\end{array}$ & \multicolumn{2}{|c|}{$\begin{array}{l}\text { Mean for } \\
\text { Control }\end{array}$} & $\begin{array}{l}\text { Stand. } \\
\text { Dev. }\end{array}$ & $\begin{array}{l}\text { Mean for } \\
\text { Experimental }\end{array}$ & $\begin{array}{l}\text { Stand. } \\
\text { Dev. }\end{array}$ & $\begin{array}{c}\text { Mean } \\
\text { Diff. }\end{array}$ & t-value & $\underline{p}$ \\
\hline \multicolumn{9}{|c|}{ NK Cells CD16+CD56+ \% (0-24\%) } \\
\hline Baseline \#1 & $(n=2)$ & 3.500 & $2.121(n=5)$ & 3.000 & 6.284 & -9.500 & -1.992 & .103 \\
\hline Measure \#2 & $(n=1)$ & 6.000 & $(n=3)$ & 12.000 & 0.000 & -6.000 & - & - \\
\hline Measure \#3 & $(n=2)$ & 6.000 & $1.414(n=4)$ & 16.250 & 6.500 & -10.250 & -2.086 & .105 \\
\hline \multicolumn{9}{|c|}{ NK Cells CD16+CD56+ \# (0.000-0.646 b/L) } \\
\hline Baseline \#1 & $(n=2)$ & 7.500 & $4.950(n=5)$ & 0.228 & 0.166 & -0.153 & -1.215 & .279 \\
\hline Measure \#2 & $(n=1)$ & 7.000 & $(n=3)$ & 0.146 & 6.658 & -7.660 & -0.997 & .424 \\
\hline Measure \#3 & $(n=2)$ & 7.000 & $4.243(n=4)$ & 0.190 & 8.206 & -0.120 & -1.868 & .135 \\
\hline \multicolumn{9}{|l|}{ Target Cells } \\
\hline Baseline \#1 & $(n=2)$ & 1.120 & $2.828(n=5)$ & 2.552 & 2.060 & -1.432 & -0.929 & .396 \\
\hline Measure \#2 & $(n=1)$ & 4.8700 & $(n=3)$ & 1.336 & 0.263 & 3.533 & 12.950 & $.006^{*}$ \\
\hline Measure \#3 & $(n=2)$ & 1.260 & $0.226(n=4)$ & 3.317 & 4.012 & -2.057 & -0.683 & .532 \\
\hline \multicolumn{9}{|c|}{ Efrector Cells } \\
\hline Baseline \#l & $(n=2)$ & 25.215 & $13.230(n=5)$ & 13.924 & 15.398 & 1.291 & 0.900 & .409 \\
\hline Measure \#2 & $(n=1)$ & 30.130 & $(n=3)$ & 29.163 & 8.234 & 0.966 & 0.100 & .929 \\
\hline Measure \#3 & $(n=2)$ & 38.880 & $11.469(n=4)$ & 24.487 & 26.345 & 14.392 & 0.706 & .519 \\
\hline \multicolumn{9}{|c|}{ NK Assay 40:1 \% death } \\
\hline Baseline \#l & $(n=2)$ & 14.095 & $3.627(n=5)$ & 35.378 & 27.623 & -21.283 & -1.027 & .351 \\
\hline Measure $t^{\prime} \cdot 2$ & $(n=1)$ & 10.930 & $(n=3)$ & 16.436 & 2.822 & -5.506 & -1.690 & .233 \\
\hline Measure \#3 & $(n=2)$ & 14.560 & $0.707(n=4)$ & 23.582 & 20.618 & -9.022 & -0.583 & .591 \\
\hline \multicolumn{9}{|c|}{ NK Assay 20:1 \% death } \\
\hline Baseline \#1 & $(n=2)$ & 7.710 & $1.569(n=5)$ & 29.562 & 21.865 & -21.852 & -1.335 & .089 \\
\hline Measure \#2 & $(n=1)$ & 6.430 & $(n=3)$ & 9.373 & 2.716 & -2.943 & -0.938 & .447 \\
\hline Measure \#3 & $(n=2)$ & 8.645 & $0.629(n=4)$ & 14.482 & 13.461 & -5.837 & -0.578 & .594 \\
\hline \multicolumn{9}{|c|}{ NK Assay 10:1 } \\
\hline Baseline \#1 & $(n=2)$ & 5.115 & $0.318(n=5)$ & 14.804 & 11.460 & -9.689 & -1.130 & .310 \\
\hline Measure \#2 & $(n=1)$ & 2.710 & $(n=3)$ & 5.906 & 1.699 & -3.196 & -1.629 & .245 \\
\hline Measure \#3 & $(n=2)$ & 3.645 & $0.487(n=4)$ & 7.595 & 5.059 & -3.950 & -1.039 & .357 \\
\hline \multicolumn{9}{|c|}{ NK Assay 5:1 } \\
\hline Baseline \#l & $(n=2)$ & 3.175 & $1.888(n=5)$ & 7.556 & 6.427 & -4.381 & -0.901 & .409 \\
\hline Measure \#2 & $(n=1)$ & 0.000 & $(n=3)$ & 3.816 & 1.301 & -3.816 & -2.540 & .126 \\
\hline $\begin{array}{l}\text { Measure \#3 } \\
\text { (*significance = }\end{array}$ & $\begin{array}{l}(n=2) \\
<<.05)\end{array}$ & 2.765 & $0.756(n=4)$ & 3.337 & 2.583 & -0.572 & -0.291 & .785 \\
\hline
\end{tabular}


(Continued)

\begin{tabular}{|c|c|c|c|c|c|c|c|c|}
\hline $\begin{array}{l}\text { Test \& \# } \\
\text { (Ref. Range) }\end{array}$ & & $\begin{array}{l}\text { lean for } \\
\text { control }\end{array}$ & $\begin{array}{l}\text { Stand. } \\
\text { Dev. }\end{array}$ & $\begin{array}{l}\text { for } \\
\text { erimental }\end{array}$ & $\begin{array}{l}\text { Stand. } \\
\text { Dev. }\end{array}$ & $\begin{array}{r}\text { Mean } \\
\text { Diff. } \\
\end{array}$ & t-value & D \\
\hline \multicolumn{9}{|l|}{ Lytic Units } \\
\hline Baseline \#l & $(n=2)$ & 15.000 & $4.242(n=5)$ & 78.400 & 98.647 & -63.400 & -0.859 & .430 \\
\hline Measure \#2 & $(n=1)$ & 1.000 & $(n=3)$ & 18.000 & 5.196 & -17.000 & -2.833 & .105 \\
\hline Measure \#3 & $(n=2)$ & 13.500 & $2.121(n=4)$ & 30.250 & 34.101 & -16.750 & -0.654 & .549 \\
\hline \multicolumn{9}{|c|}{ PHA $12.5(49,393-21,015 \mathrm{cpm})$} \\
\hline Baseline \#1 & $(n=2)$ & 507.350 & $109.389(n=4)$ & 464.500 & 460.498 & 42.850 & 0.123 & .908 \\
\hline Measure \#2 & $(n=1)$ & 306.600 & $(n=3)$ & 455.033 & 500.092 & -148.433 & -0.257 & $.82 i$ \\
\hline Measure \#3 & $(n=2)$ & 273.700 & $148.351(n=4)$ & 690.950 & 573.286 & -417.250 & -0.960 & .392 \\
\hline \multicolumn{9}{|c|}{ PHA $3.125(31,140-11,850 \mathrm{cpm})$} \\
\hline Baseline \#1 & $(n=2)$ & 242.850 & $165.816(n=4)$ & 190.700 & 233.384 & 52.150 & 0.276 & .796 \\
\hline Measure \#2 & $(n=1)$ & 27.900 & $(n=3)$ & 349.000 & 414.621 & -321.100 & -0.671 & .571 \\
\hline Measure \#3 & $(n=2)$ & 141.400 & $119.783(n=4)$ & 249.600 & 184.172 & -108.200 & -0.733 & .504 \\
\hline \multicolumn{9}{|c|}{ PHA $1.56(15,310-696 \mathrm{cpm})$} \\
\hline Baseline \#1 & $(n=2)$ & 176.450 & $178.403(n=4)$ & 46.925 & 52.108 & 129.525 & 1.006 & .487 \\
\hline Measure \#2 & $(n=1)$ & 13.500 & $(n=3)$ & 171.566 & 220.172 & -158.066 & -0.622 & .598 \\
\hline Measure \#3 & $(n=2)$ & 66.150 & $45.891(n=4)$ & 133.675 & 89.663 & -67.525 & -0.963 & .390 \\
\hline \multicolumn{9}{|c|}{ IL-4 (normal 0.6-450 pg/ml) } \\
\hline Baseline \#1 & $(n=2)$ & 0.5200 & $0.141 \quad(n=5)$ & 1.034 & 1.134 & -0.514 & -0.604 & .572 \\
\hline Measure \#2 & $(n=1)$ & 0.840 & $(n=3)$ & 0.846 & 0.341 & -6.666 & -0.017 & .988 \\
\hline Measure \#3 & $(n=2)$ & 0.445 & $0.134(n=4)$ & 0.560 & 0.171 & -0.115 & -0.815 & .461 \\
\hline \multicolumn{9}{|c|}{ L-6 (0.6-450 pg/ml) } \\
\hline Baseline \#I & $(n=1)$ & 4.800 & $(n=5)$ & 3.870 & 4.852 & 0.930 & 0.175 & .870 \\
\hline Measure \#2 & $(n=1)$ & 4.320 & $(n=3)$ & 1.773 & 0.190 & 2.546 & 11.602 & $.007^{*}$ \\
\hline Measure \#3 & $(n=2)$ & 2.435 & $1.690(n=4)$ & 6.967 & 9.264 & -4.532 & -0.649 & .552 \\
\hline \multicolumn{9}{|c|}{ L-12 (normal: 0 pg/mL) } \\
\hline Baseline \# I & $(n=2)$ & 1.065 & $1.506(n=5)$ & 0.964 & 1.761 & 0.101 & 0.070 & .947 \\
\hline Measure \#2 & $(n=1)$ & 0.000 & $(n=3)$ & 1.667 & 2.887 & -1.666 & -0.500 & .667 \\
\hline Measure \#3 & $(n=2)$ & 0.000 & $0.000(n=4)$ & 2.100 & 2.545 & -2.100 & -1.100 & .333 \\
\hline
\end{tabular}

(*significance $=p<.05)$ 